\title{
VERSATILE CHROMIUM-DOPED ZINC SELENIDE INFRARED LASER SOURCES
}

\author{
Dissertation \\ Submitted to \\ The School of Engineering of the \\ UNIVERSITY OF DAYTON \\ In Partial Fulfillment of the Requirements for \\ The Degree of \\ Doctor of Philosophy in Electro-Optics \\ by

\section{Patrick A. Berry} \\ University of Dayton \\ Dayton, Ohio \\ May, 2010
}




\section{VERSATILE CHROMIUM-DOPED ZINC SELENIDE INFRARED LASER SOURCES}

\section{APPROVED BY:}

Peter E. Powers, Ph.D.

Advisory Committee Chairman

Professor, Physics
Joseph W. Haus, Ph.D.

Committee Member

Professor and Director, ElectroOptics
Kenneth L. Schepler, Ph.D.

Committee Member

Principal Scientist, Sensors Directorate, Air Force Research Laboratory

Malcolm W. Daniels, Ph.D.

Associate Dean

School of Engineering
Andrew Sarangan, Ph.D.

Committee Member

Associate Professor, Electro-Optics
Tony E. Saliba, Ph.D.

Dean, School of Engineering 


\section{Abstract \\ VERSATILE CHROMIUM-DOPED ZINC SELENIDE INFRARED LASER SOURCES}

Name: Berry, Patrick A.

University of Dayton

Advisor: Peter E. Powers, PhD

The atmospheric transmission windows of 2-5 and 8-12 $\mu \mathrm{m}$, coupled with organic and other chemical absorption lines occurring throughout this middle-infrared (mid-IR) wavelength region give rise to a wide variety of medical, scientific, commercial and military applications. Communications, remote sensing, IR countermeasures, laser surgery and non-invasive imaging are just a few of the drivers of high-power solid-state mid-IR laser development. These laser sources must be versatile enough to operate in a variety of temporal modes from continuous wave $(\mathrm{CW})$ all the way to ultrashort pulse while still being widely tunable for wavelength agility. All of this is required at ever increasing power output levels while conforming to size, weight and power consumption limitations under harsh operating environmental conditions.

Chromium-doped zinc selenide $\left(\mathrm{Cr}^{2+}: \mathrm{ZnSe}\right)$ lasers operating in the 2-3 $\mu$ m region are excellent candidates to help fill these vital roles. As a transition-metal doped II-VI 
chalcogenide, $\mathrm{Cr}^{2+}: \mathrm{ZnSe}$ has a number of positive advantages over existing laser sources. Development and power scaling of these lasers however, has been hampered by thermal issues which have so far limited the ability of these lasers to be applied to systems-level development.

This work presents research into the nature and mitigation of these critical thermal issues in development of versatile $\mathrm{Cr}^{2+}: \mathrm{ZnSe}$ laser sources. Advanced models for thermal and laser performance are developed and used to design optimally configured laser systems. Among other advances for this material, $>10 \mathrm{~W} \mathrm{CW}$ output from a $\mathrm{Cr}^{2+}: \mathrm{ZnSe}$ oscillator and master-oscillator / power amplifier systems producing multi-watt, widely tunable power levels are demonstrated. 


\section{Dedication}

This work is dedicated to two true friends:

Saffar Arjmandi, my friend and partner-in-crime from whom I learned many valuable lessons, most of all what it means to soldier on in the face of adversity. RLTW

Christopher Woody, who always had my back and kept me going through the worst of times. 


\section{Acknowledgements}

I would like to graciously acknowledge help and support of the following:

Dr. Kenneth Schepler, Capt. Torrey Wagner, John Carr, John McCurdy, Charles Phelps, Jonathan Evans, Branden King, and Dr. Rita Peterson from the Air Force Research Lab, Sensors Directorate, AFRL/RYJW

Dr. Peter Powers from the University of Dayton

Sergey Mirov, Igor Moskalev and Vladimir Fedorov from the University of Alabama Birmingham

Funding support was provided by the Air Force Office of Scientific Research and the Air Force Research Lab, Sensors Directorate 


\section{Table of Contents}

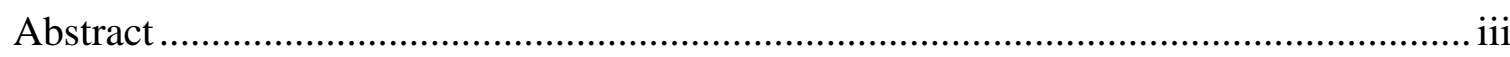

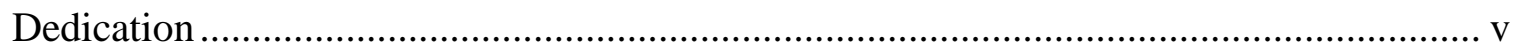

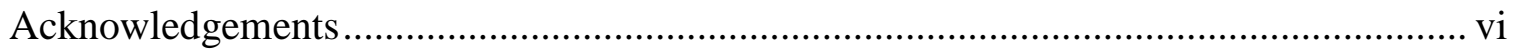

Table of Contents ...................................................................................................... vii

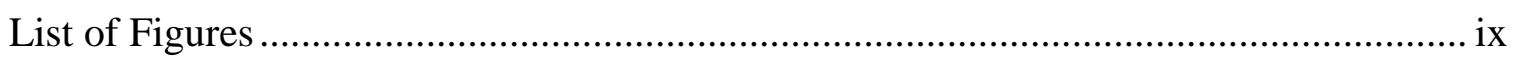

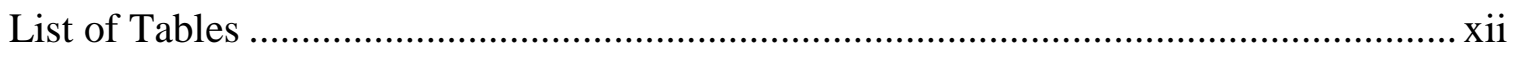

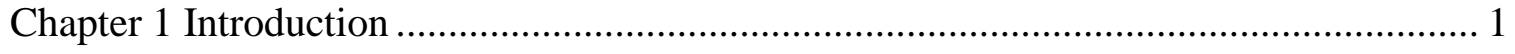

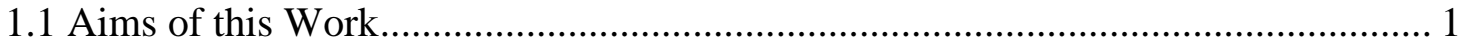

1.2 Dissertation Outline and Relevant Publications and Presentations ......................... 2

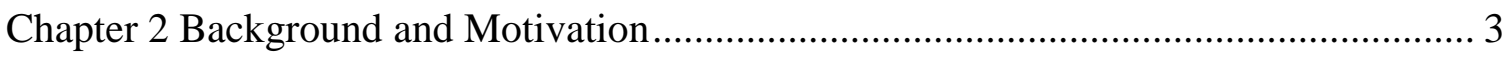

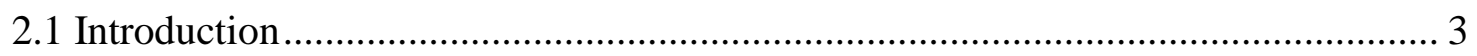

2.2 Early Solid-state Lasers ............................................................................ 5

2.3 Transition Metal Lasers .................................................................................. 5

Chapter 3 Modeling of Time-Dependent Thermal Effects ............................................ 8

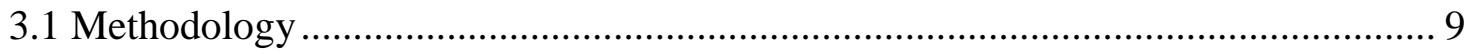

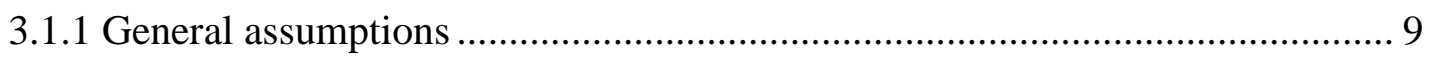

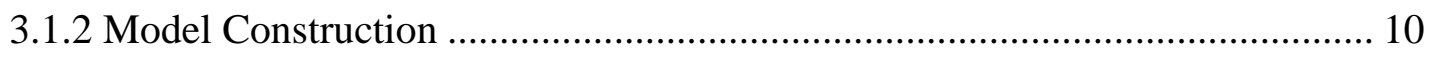

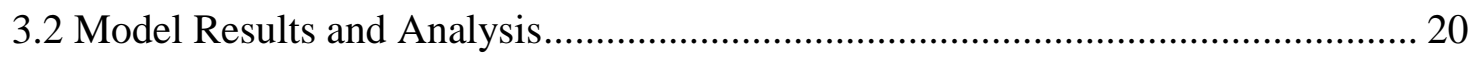

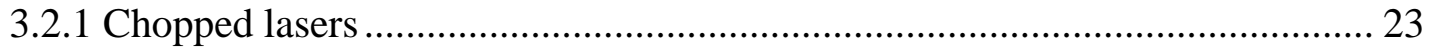

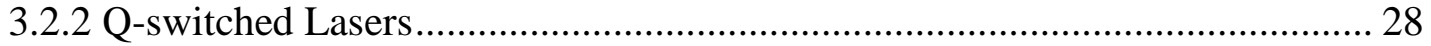

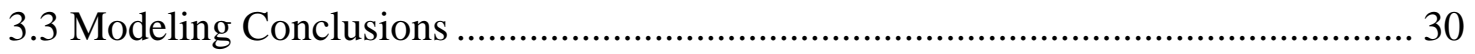

Chapter 4 Chromium-doped Zinc Selenide Fiber Laser .............................................. 32

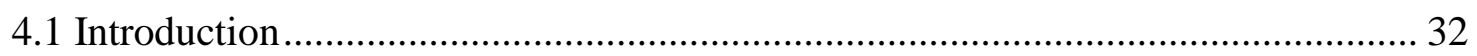




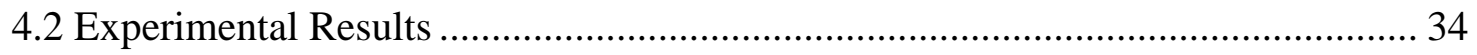

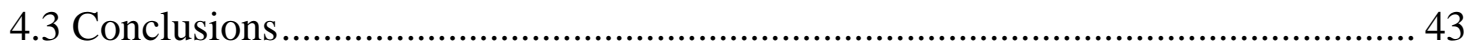

Chapter 5 High-power, Widely-tunable $\mathrm{Cr}^{2+}: \mathrm{ZnSe}$ Lasers .......................................... 44

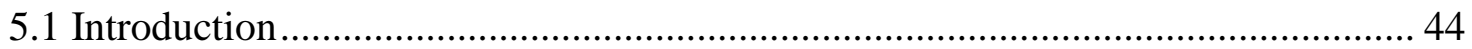

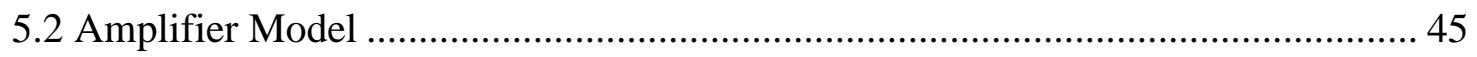

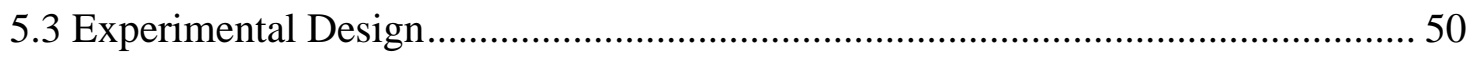

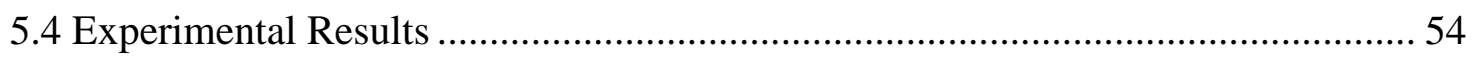

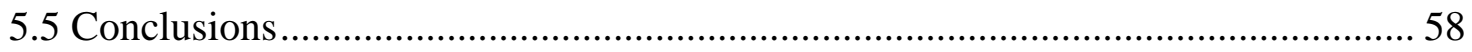

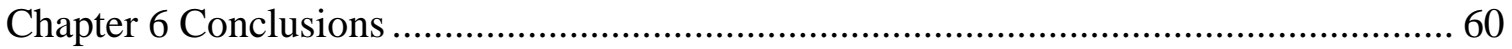

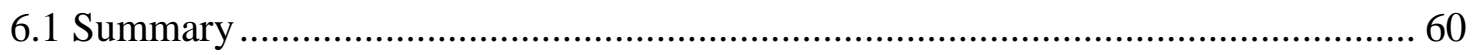

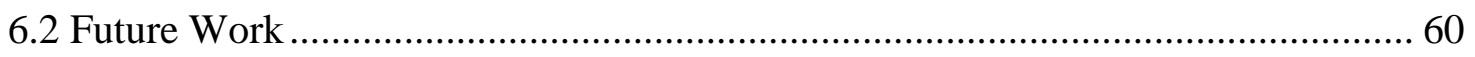

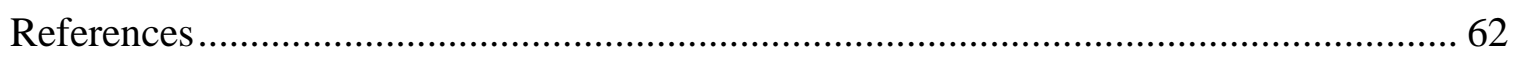

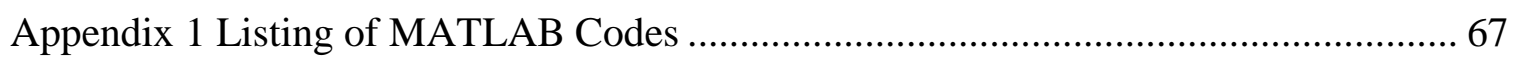

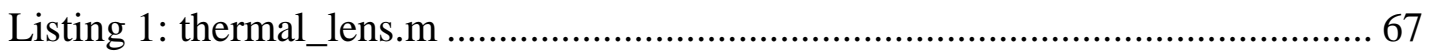

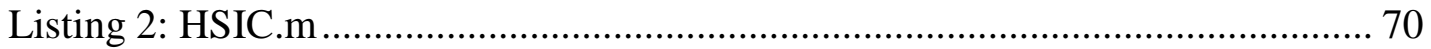

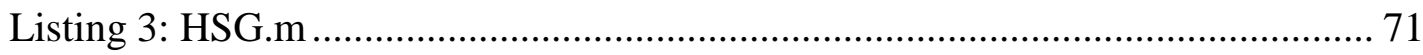

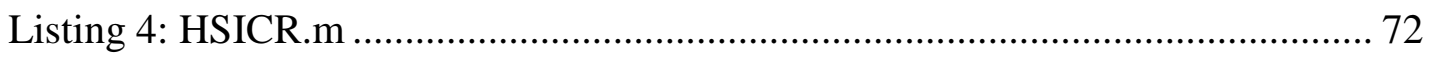

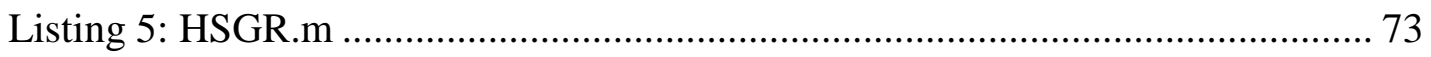

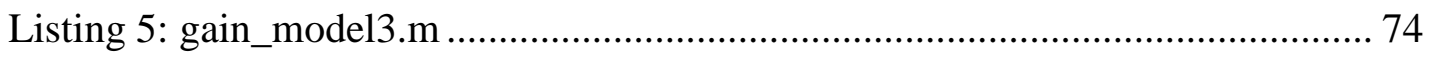

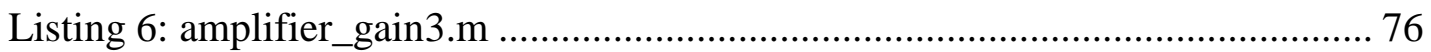

Appendix 2 LabView Control Program for Laser and Power Meter Control.................... 77

Appendix 3 Z-Cavity Alignment Procedure …………………………............................ 80 


\section{List of Figures}

Figure 1: Simulated atmospheric transmission indicating areas of interest for mid-IR laser

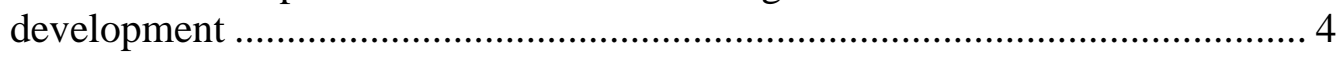

Figure 2: Gaussian beam radial distributions (update y label) .................................... 10

Figure 3: Basic face cooled design translation to model geometry ................................ 11

Figure 4: Generation of a time dependent square wave. (a) sine function, (b) $50 \%$ duty cycle smoothed Heaviside function, (c) sine function offset by $\cos (0.2 \pi)=$ 0.628 , (d) $20 \%$ duty cycle smoothed Heaviside function

Figure 5: The z-average temperature at the $x-y$ center point for the different time increments over a total of $4 \mathrm{~ms}$ 15

Figure 6: The $\mathrm{z}$-average temperature at the $\mathrm{x}-\mathrm{y}$ center point comparison for two different time increments for a total of $8 \mathrm{~ms}$ 16

Figure 7: Comparison of single continuous model run to combine discrete runs showing acceptability of using discrete model runs for computational convenience..... 17

Figure 8: $16 \mathrm{~ms}$ modeling run at $1 \mu \mathrm{s}$ increments to determine asymptotic behavior...... 18

Figure 9: Simulation solution of $4 \mathrm{~mm}$ diameter, $1 \mathrm{~mm}$ thick face cooled thin disk pumped with a Gaussian $(\mathrm{n}=1)$ beam with $\sigma=0.4 \mathrm{~mm}$. The temperature ranges from 293 (dark) to 306 (light) .................................................... 21

Figure 10: Temperature gradient surface for previous $4 \mathrm{~mm}$ face cooled thin disk solution 21

Figure 11: Center point z-average temperature for $1 \mathrm{kHz}$ PRF at varying duty cycles.... 24

Figure 12: Radial temperature gradient for $1 \mathrm{kHz}$ PRF at varying duty cycles............... 25

Figure 13: Experimental results for $1 \mathrm{kHz}$ PRF chopped laser with varying duty cycles. Pump power is average power............................................................. 26

Figure 14: Experimental results for fixed PRF and duty cycles for varying heat sink

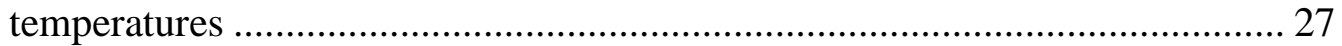

Figure 15: Center point $z$-average temperature for $15 \%$ duty cycle with varying PRF ... 29

Figure 16: Radial temperature gradient for $15 \%$ duty cycle at varying PRFs. 30 


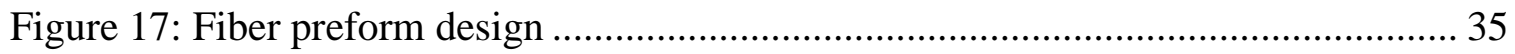

Figure 18: Preform remaining after first fiber draw attempt ......................................... 35

Figure 19: Fiber preform assembly, step one.......................................................... 36

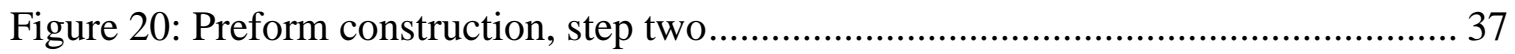

Figure 21: Exploded preform from second fiber draw attempt ....................................... 38

Figure 22: Polished and cleaved preform, ready for spectroscopy …………………...... 39

Figure 23a: Decay pulses from preform; 7b: Lifetime measurement of core .................... 40

Figure 24: Spectroscopy results for different postions on fiber preform ........................... 41

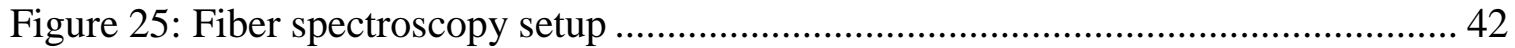

Figure 26: New preform designs a) solid, short core and b) long, small diameter

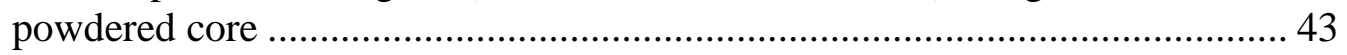

Figure 27: $\mathrm{Cr}^{2+}$ energy levels in $\mathrm{Cr}^{2+}: \mathrm{ZnSe}$ relative to the band gap [56] ....................... 46

Figure 28: Example model predictions showing (left) input signal growth and pump absorption over the crystal length for $5 \mathrm{~W}$ input signal and $5 \mathrm{~W}$ pump, both at $80 \mu \mathrm{m}$ spot size and (right) amplification comparisons for 5 and $10 \mathrm{~W}$ input signal powers with varying spot size as a function of pump power. 50

Figure 29: Astigmatically-compensated Z-cavity design which can use either a single collimated-leg design (dashed) or a double-collimated leg design. $\mathrm{OC}=$ output coupler, $\mathrm{LP}=$ laser pump lens, $\mathrm{FE}=$ functional element. 51

Figure 30: Output power vs incident pump power for single-leg Z-cavity. The average slope efficiency is $\sim 40 \%$. The error bars show the minimum and maximum of 1000 measurements of output power per second at each data point. The output power oscillation and random non-linearity of the curve are attributed to thermal lensing instability and the random pump beam distortions caused by atmospheric water vapor absorption of the pump radiation. . 52

Figure 31: A) Normal-incidence $L$-cavity scheme which can use either an HR curved mirror (M1) or a combination of planar HR (M2) and positive lens (L1). B) Power amplifier design where $\mathrm{L}_{\mathrm{MO}}$ and $\mathrm{L}_{\mathrm{PA}}$ are chosen for optimal overlap of pump and signal beams. 53

Figure 32: Power scaling ability of MOPA configurations. Experimental data (discrete points) is compared with model predictions for $L_{a^{-}}$and $Z$-cavities, both with $100 \mu \mathrm{m}$ spot sizes. 55

Figure 33: Output spectrum of free-running $L_{b}$-cavity as a function of output power showing a shift to higher wavelengths at increased power. ............................. 56

Figure 34: Tunable output power of $Z$-cavity with representative linewidth of output.... 57

Figure 35: Single-frame excerpt (left) from video showing near-field beam profile changes of $L_{b}$-cavity as a function of decreasing pump power. Movie starts with maximum pump power and ends at zero pump power. Transitions between different modes cause instability in power output (right)................... 58 
Figure 36: Control panel for IPG 150W Tm:Fiber laser control ................................. 78

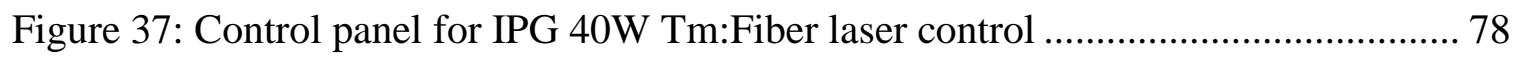

Figure 38: Control panel for power meter automated data collection (left) and for automated laser control with data collection (right) ................................. 79

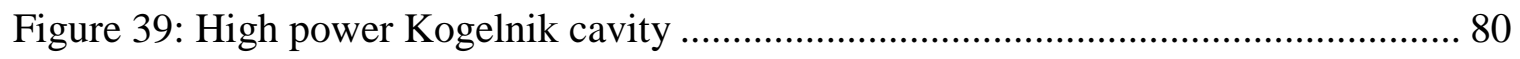




\section{List of Tables}

Table 1: Dissertation related presentations and publications......................................... 2

Table 2: Material properties of various laser host materials[46] .................................... 7

Table 3: Thermal Lensing Power at $1 \mathrm{kHz}$ PRF, $1 \mathrm{~W}$ peak power, Chopped Laser ........ 25

Table 4: Thermal Lensing Power at $1 \mathrm{kHz}$ PRF, $1 \mathrm{~W}$ peak power, Q-switched Laser .... 30 


\section{Chapter 1}

\section{Introduction}

\subsection{Aims of this Work}

Laser sources operating in the middle infrared (mid-IR) wavelength region from 2-12 $\mu \mathrm{m}$ are necessary for a number of military, scientific and other types of applications. These applications are driven in general by both the atmospheric transmission bands of $2-5 \mu \mathrm{m}$ and $8-12 \mu \mathrm{m}$ as well as the locations of chemical lines throughout the region. The very nature of applications such as remote chemical sensing, infrared countermeasures (IRCM), laser communications and laser surgery drive other laser operational requirements such as output power, tunability, beam quality and room temperature operations to name just a few. The broad goal of this research was to understand, design and construct useful laser sources with emission in this important wavelength range. This work builds on years of basic research in this area but also incorporates requirementsdriven feedback from systems-level developments with the end goal of accomplishing a broader understanding of the science involved while still producing useful end products.

The approach used to produce coverage of these broad bands was to develop high power, tunable laser direct laser sources to cover the 2-3 $\mu \mathrm{m}$ region and then use nonlinear devices to convert this power out into 3-5 $\mu \mathrm{m}$ and 8-12 $\mu \mathrm{m}$. This work focuses on the first piece of that puzzle: developing versatile direct laser sources in the $2-3 \mu \mathrm{m}$ 
wavelength range. Modeling of system behavior was used whenever possible to save research time and enhance the probability of success by prioritizing research alternatives based on model results.

\subsection{Dissertation Outline and Relevant Publications and Presentations}

This dissertation is broadly broken up into chapters covering different types of $\mathrm{Cr}^{2+}: \mathrm{ZnSe}$ lasers which were investigated under the umbrella of this work. Chapter 2 will cover the background of high-power mid-IR lasers as well as a review of relevant research and our previous work in this area. Chapter 3 presents modeling of thin-disk lasers undertaken in an attempt to better understand the effect of different pumping sources, both $\mathrm{CW}$ and pulsed, on the thermal load of the material. Chapter 4 covers work done to develop a $\mathrm{Cr}^{2+}: \mathrm{ZnSe}$ fiber laser. Chapter 5 showcases work done to model and build a high power, tunable, thin-slab $\mathrm{Cr}^{2+}: \mathrm{ZnSe}$ master oscillator / power amplifier system. The publications and presentation associated with each chapter can be seen in Table 1.

Table 1: Dissertation related presentations and publications

\section{Chapter}

1 Introduction

2 Background and Motivation

3 Time-dependent Modeling

$4 \mathrm{Cr}^{2+}: Z n S e$ Fiber Laser

$5 \mathrm{Cr}^{2+}: \mathrm{ZnSe}$ High-power Systems

\section{Presentations and/or Publications}

SPIE Newsroom Article [1] ${ }^{\mathrm{b}}$

IEEE Journal of Selected Topics in Quantum Electronics [2] $]^{\mathrm{b}}, 2006$ Phot. West presentation ${ }^{\mathrm{a}}$, SPIE Proceedings [3] ${ }^{\mathrm{a}}$

2009 ASSP presentation, [4] $]^{\mathrm{b}}, 2010$ Phot. West presentation, SPIE proceedings [5] ${ }^{\mathrm{a}}, 2010$ Optics Express [6] ${ }^{\mathrm{a}, \mathrm{c}}, 2010$ CLEO presentation [7] ${ }^{\mathrm{a}, \mathrm{c}}$

${ }^{\mathrm{a}}$ Presenter and/or primary author; ${ }^{\mathrm{b}}$ co-author; ${ }^{\mathrm{c}}$ submitted 


\section{Chapter 2}

\section{Background and Motivation}

\subsection{Introduction}

As mentioned in Chapter 1, the 2-12 $\mu \mathrm{m}$ wavelength range is one in which a number of important applications occur. Due to the absorption characteristics of its constituent gasses, the atmosphere has two broad ranges from 2-5 $\mu \mathrm{m}$ and 8-12 $\mu \mathrm{m}$ in which high transmission occurs (Figure 1). Applications such as laser ranging and imaging, target identification and laser-based communications which rely upon sending and receiving laser pulses leverage these high-transmission windows in order to extend operating distances. Anti-aircraft missiles utilize these windows to maximize their ability to detect heat from target aircraft either generated by engine combustion or by friction between the aircraft and the atmosphere. This threat drives the requirements for lasers in these ranges for use in infrared countermeasures for aircraft self-protection. 


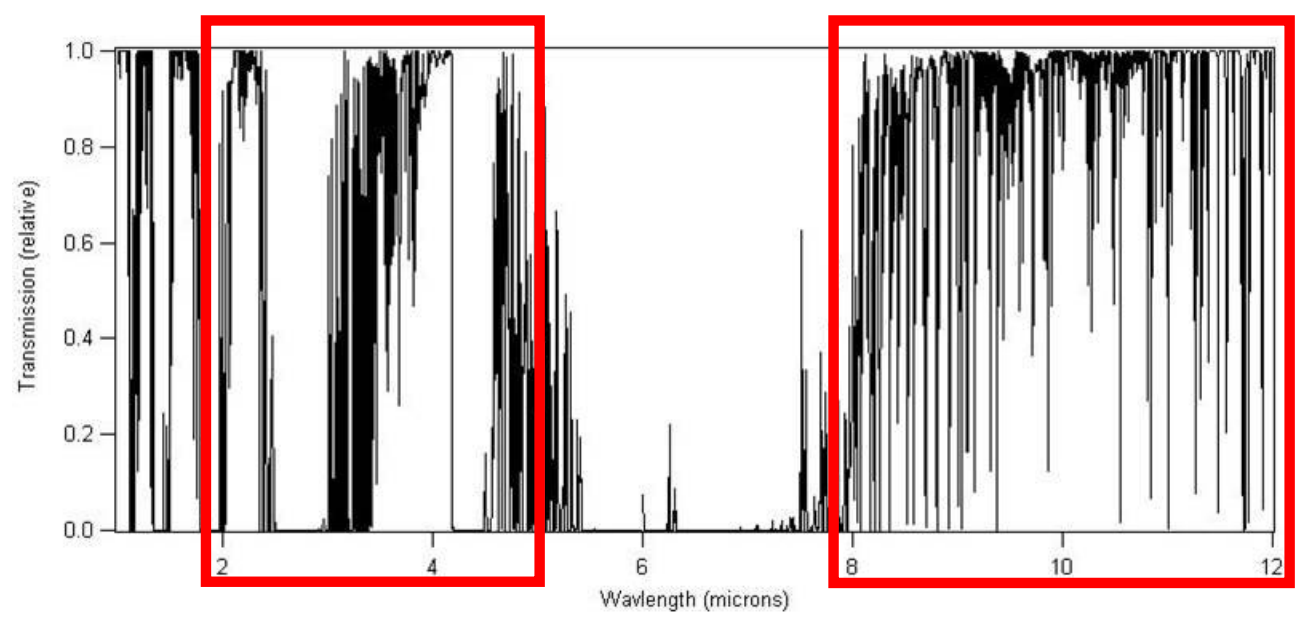

Figure 1: Simulated atmospheric transmission indicating areas of interest for mid-IR laser development

In addition to the atmospheric absorption characteristics, there are a number of organic and other molecular absorptions which occur in this region. These absorption features drive applications such as remote chemical sensing in which lasers are used to probe areas from a safe or practical distance to determine the makeup of unknown samples. Similar absorption properties of tissues and proteins drives medical applications such as laser tissue cutting, ablation and welding [8,9].

All of these applications have specific laser operating requirements. Temporally, the laser may need to operate anywhere from continuous wave (CW), to long pulse (ms), to short pulse (ns), all the way down to ultrashort pulse (ps, fs). Many of the applications require the ability to tune the wavelength through tens of nanometers or more in short time periods, allow for frequency modulation or multiplexing or select specific absorption features. The end result of all of the above is a broad requirements field which necessitates multiple lasers to ensure coverage. What is really needed is a laser versatile enough to do the work of many lasers. 


\subsection{Early Solid-state Lasers}

Townes and Schawlow laid the groundwork for the first solid state laser in 1958 with their description of infrared and optical masers [10], which lead to Maiman's demonstration of the first ruby (chromium-doped sapphire) laser in 1960 [11]. Attention quickly turned to rare earth-doped materials including Nd:YAG [12], Nd:YALO [13], among many others. Now Nd-based lasers are ubiquitous with output at $1.064 \mu \mathrm{m}$ along with many possible nonlinear-converted wavelengths. These same hosts have also been demonstrated with a wide variety of other rare earths such as erbium [14], and holmium [15] just to name a few [16]. Rare earth lasers fill a number of vital roles in commercial, medical and military needs; however their tunability and operating wavelengths in the mid-IR range of interest are limited by their low crystal field interactions with their host materials[17]. This arises from the fact that the $f$ orbitals in which the valence electrons lie are shielded from host interactions by filled outer orbitals $[18,19]$.

\subsection{Transition Metal Lasers}

Transition metal (TM) lasers, in contrast to the rare earths, have always been found to have broad tunability (with the exception of the ruby laser). This comes from the fact that the active electrons in these ions lie in partially-filled $d$ orbitals which are not shielded by filled outer shells. This allows these ions to interact strongly with their host crystal lattices giving broad emission and absorption spectra. Early TM lasers were demonstrated with $\mathrm{Ni}^{2+}[20]$ and $\mathrm{Co}^{2+}[21]$ but the tunability of these lasers was limited by excited state absorption (ESA) and were only ran efficiently at cryogenic temperatures [22-24], a requirement limiting the size, weight and power of the lasers. This inability to 
run efficiently at room temperatures is due to the decreasing fluorescence lifetime associated with increasing non-radiative transition rates at higher temperatures.

The real breakthrough in mid-IR TM lasers came in 1995 when a whole new class of TM-doped II-VI chalcogenide materials was reported by researchers at Lawrence Livermore National Laboratory [25-27]. Room temperature lasing has been reported in a number of different transition-metal doped II-VI materials including $\mathrm{Cr}^{2+}: \mathrm{ZnSe}$ [27-29], $\mathrm{Cr}^{2+}: \operatorname{ZnS}[27,28,30], \mathrm{Cr}^{2+}: \mathrm{Cd}_{1-\mathrm{x}} \mathrm{Mn}_{\mathrm{x}} \mathrm{Te}[31,32]$, and $\mathrm{Cr}^{2+}: \mathrm{CdSe}[33,34]$ crystals. Much of the focus however, has been on $\mathrm{Cr}^{2+}: \mathrm{ZnSe}$ which has the desirable properties of broad tunability $[35,36]$ and absorption bands, a large gain cross section, no excited state absorption, and finally high thermal conductivity, infrared transparency and quantum efficiency at room temperature. The versatility of this laser material has been shown through fiber laser pumping [37], diode pumping [38], continuous wave (CW) lasing with efficiency exceeding $60 \%$ [39, 40], output power levels exceeding $12 \mathrm{~W}$ [4], gain switched lasing with average output power of up to $18.5 \mathrm{~W}$ [41], as well as narrowlinewidth operation [42]. Only relatively recently have higher-quality $\mathrm{Cr}^{2+}: \mathrm{ZnS}$ crystals been fabricated and because of this, these types of lasers have not been as extensively demonstrated. However, they have been shown to be capable of multi-watt output [43] and can have some advantages over $\mathrm{Cr}^{2+}: \mathrm{ZnSe}$ with respect to physical properties [44, 45]. Power scaling of chromium lasers has long been hampered by the problem of thermal lensing due to the high thermo-optic coefficients, compared to other materials in Table 2: Material properties of various laser host materials of $\mathrm{ZnS}\left(46 \times 10^{-6} 1 / \mathrm{K}\right)$, $\mathrm{ZnSe}$ (70 x $10^{-6} 1 / \mathrm{K}$ ), CdSe and CdTe (both $\sim 100 \times 10^{-6} 1 / \mathrm{K}$ ) [46]. Thermal lensing in the gain material leads to optical damage or cavity instability which limits the maximum 
achievable output power. Thermal lensing occurs when variations in the refractive index across the material, arising from thermal gradients, alter the phase profile of light passing through the material, effectively acting as a lens. This can cause cavity instability as well as material damage from focusing at high powers. The thermal gradients that cause thermal lensing typically come from excess heat generated by pumping, either through the quantum defect or from non-radiative losses [2].

Table 2: Material properties of various laser host materials[46]

\begin{tabular}{llllll}
\hline Property & ZnSe & ZnS & CdSe & CdTe & Sapphire $\left(\mathbf{A l}_{2} \mathbf{O}_{3}\right)$ \\
\hline$\kappa\left(\mathrm{Wm}^{-1} \mathrm{~K}^{-1}\right)$ & 18 & 17 & 4 & 7.5 & 27 \\
$\operatorname{dn} / \mathrm{dT}\left(10-{ }^{6} / \mathrm{K}\right)$ & 70 & 46 & 98 & 100 & 12 \\
$n\left(\sim \lambda_{\text {lasing }}\right)$ & 2.45 & 2.27 & 2.47 & 2.7 & 1.76 \\
$\begin{array}{l}\text { Hardness } \\
\text { (Knoop) }\end{array}$ & 160 & 120 & 70 & 45 & 1800,2200 \\
\hline
\end{tabular}

These thermal issues can be addressed through appropriate choices of crystal geometry, a longer wavelength pump source and heat removal, all of which help to reduce the thermal load in the gain element [2]. A number of designs for thermal lens mitigation resonators are found in the literature and include the fiber waveguide-based laser, ziz-zag mode slab laser and the thin disk laser. While some progress has been made in addressing this problem using thin disk laser designs, there is still room for improvement [47]. 


\section{Chapter 3}

\section{Modeling of Time-Dependent Thermal Effects}

In order to design lasers which mitigate the thermal effects arising from thermal lensing in $\mathrm{ZnSe}$, we must understand the time-evolution of the lensing. As a first step in this, a modeling system was developed that could be used to gain an understanding of the timedependence of thermal lensing as well as to help expedite the development of better laser designs. With this in mind, it was important to not only construct models with varying time-dependent variables but to also study the effects of variations in the ancillary model parameters. These extra steps were needed to decouple the modeling assumptions from the parameters to be tested and establish a baseline model from which the proposed designs can be constructed.

When modeling heat distribution and material properties, finite element analysis is the modeling method of choice. Commercially available finite element software was used to model the time dependence of thermal lensing and temperature rise in a $\mathrm{Cr}^{2+}: \mathrm{ZnSe}$ thin disk. Two general types of sources are available to use as a pump for $\mathrm{Cr}^{2+}: \mathrm{ZnSe}$ lasers, diode-pumped, Q-switched lasers such as Tm:YLF or Tm:HoYLF, or diode-pumped CW fiber lasers such as Tm:fiber, which can be modulated to allow for varying duty cycles. Both of these cases, chopped CW and Q-switched pumping, were considered. 


\subsection{Methodology}

\subsubsection{General assumptions}

To simplify the modeling process, certain assumptions were made. The laser source was assumed to be uniformly absorbed along the depth of the laser sample. In a typical single-pass absorption, one would expect pump absorption which followed Beer's law along the depth axis. In the case which we have modeled, the pump is reflected at the rear face. This, along with multiple pump passes (16 in our model) will even out the absorption at different sample depths. Heat transfer to the heat sink was assumed to experience no losses due to the interface and the heat sinks maintained a constant temperature on their surface. Lastly, conductive cooling by air was neglected such that all non-heat sink surfaces were considered to be insulating. While there will be some conductive heat loss to the air, the conductivity of air is orders of magnitude lower than copper $\left(0.024 \mathrm{Wm}^{-1} \mathrm{~K}^{-1}\right.$ vs $\left.401 \mathrm{Wm}^{-1} \mathrm{~K}^{-1}\right)[48]$.

The pump beam was assumed to have a Gaussian transverse intensity beam profile, given by

$$
f_{n}(x, y)=\exp \left[-\frac{2\left(\left(x-x_{0}\right)^{2}+\left(y-y_{0}\right)^{2}\right)^{n}}{\left(w^{2}\right)^{n}}\right]
$$

where $x_{o}$ and $y_{o}$ define the center point of the beam, $n$ is the Gaussian order, and $w$ is the beam radius at which the value of the function drops to $1 / \mathrm{e}^{2}$. The Gaussian order, $n$, could be varied to study the effects of different beam profiles, as seen in fig. In the case of our pump lasers however, $n=1$ was used throughout. 


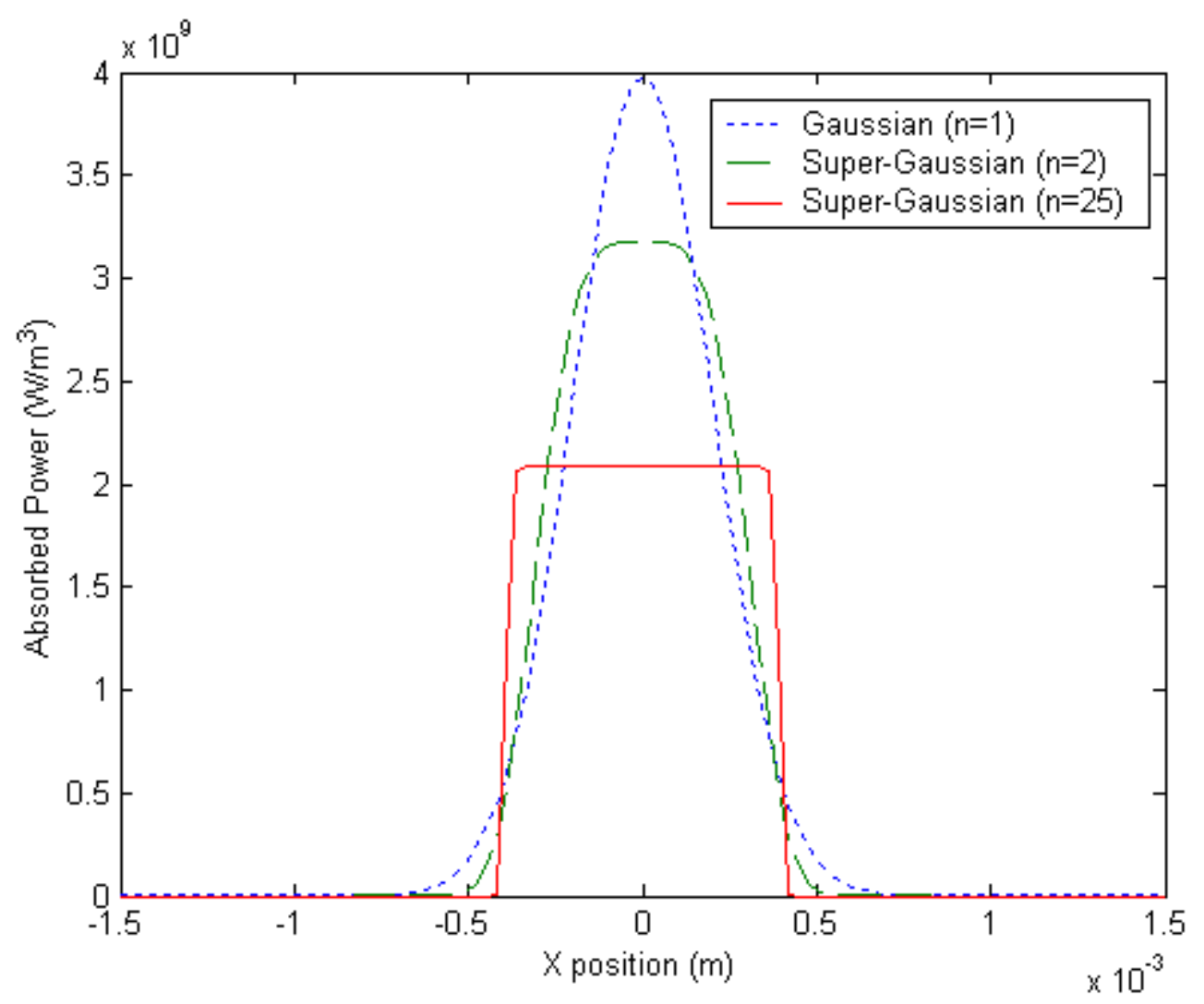

Figure 2: Gaussian beam radial distributions (update y label)

An integration factor for each of these profiles had to be calculated to normalize each one to $1 \mathrm{~W}$ of absorbed power. Custom MatLab functions (see Appendix) were used to calculate the heat source (Gaussian) and heat source integration constant for the beam profile. Two additional custom functions were used to verify that the integration constants were essentially the same for cylindrical co-ordinates. Using the results of these calculations, the integration constants were applied in the model to normalize the power to $1 \mathrm{~W}$.

\subsubsection{Model Construction}

The models were constructed using FemLab® 3.0a, version number 3.0.0.228. The first step in the model construction process was to create the model geometry using the 
program's CAD interface. In this step it is important to faithfully recreate the important elements of the design while minimizing the extraneous details. This step is important due to the fact that the complexity of the model and therefore the amount of time and memory required in solving the model are directly related to the size of the model and the number of surfaces.

\subsubsection{Model design}

A common laser design, the face-cooled thin disk, was simplified to consist of the laser material and a heat sink. Due to the general assumption of perfect heat sinks, the only part of the heat sink that needed to be included was the surface that was in direct contact with the laser material or window. To illustrate this, Figure 3 shows how the face-cooled thin disk design was 'translated' into the model geometry.
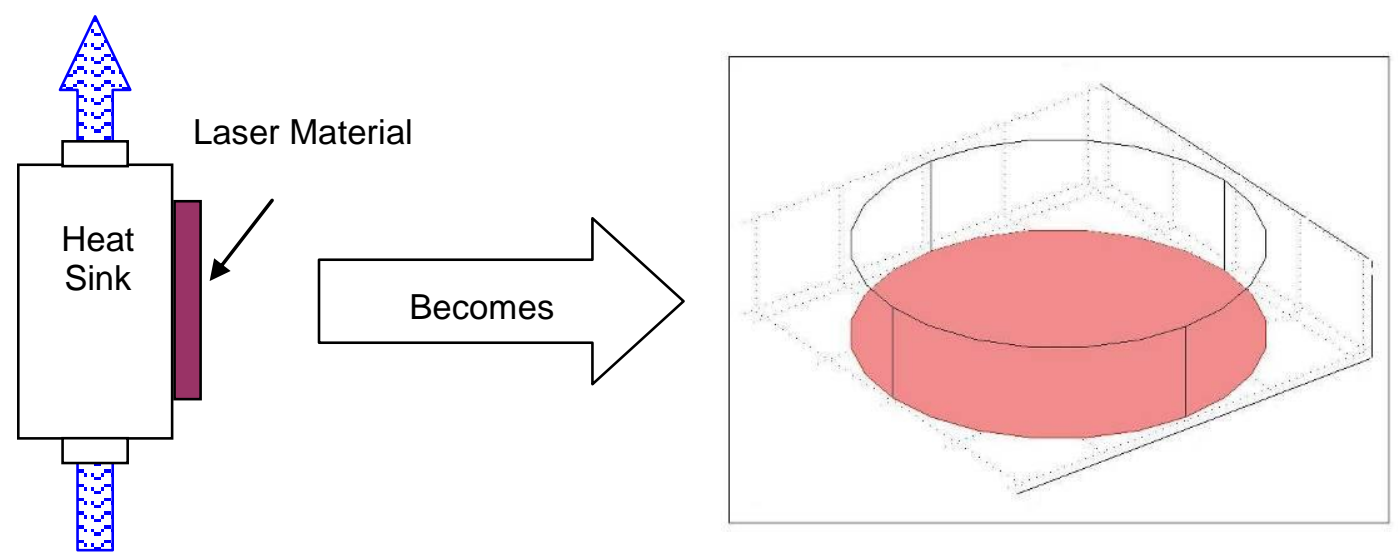

Figure 3: Basic face cooled design translation to model geometry

The next step in the model construction is to define the boundary conditions for the geometry. In general, there are three types of boundaries in this type of model. Any 
surface that touches air is considered to be an insulator such that any radiative cooling by air is neglected. Any surface that is to be considered a heat sink or that touches coolant should be set to the value of the heat sink temperature constant. All other surfaces are usually intersections of objects or subdomains and their parameters are solved for by the program.

Once the model geometry was established, boundary conditions and subdomain material parameters were set according to the material properties of the system. These parameters include the important physical properties of the host material, zinc selenide. The subdomain settings also include the heat source $Q$. This is an extremely important parameter as it not only includes the beam profile from above but also the time dependence of the pump beam.

\subsubsection{Time-dependent pump algorithm}

A method for simulating periodic pumping by a laser source was developed for use in the heat source. The variable $t$ is available for use with the time-dependent solver and can be used to create a periodic square wave pulse. This function had to have the value of 1 when the pump laser is on and 0 when it is off. The simplest method to create a square wave was to use logic functions to turn the power on and off. However, solutions generated with this method were found to be extremely inconsistent due to discontinuities of first derivates at the on/off transition times. A better way to create a periodic square wave was through the use of a smoothed step function known as a smoothed Heaviside function. This function, while still essentially the unit step function $(x>=0)$, is smoothed at the step allowing it to have a first derivative at that point. 
The Heaviside function is not a periodic function however, so it needed to operate on a periodic function to achieve the desired pulse train. To understand the algorithm better, it is necessary to look at how a Heaviside function would behave when operating on a periodic function such as a sine wave (Figure 4a). The Heaviside function will then turn on when $\sin (2 \pi t / T)>=0$ and off when $\sin (2 \pi t / T)<0$ (Figure $4 \mathrm{~b})$ where $T$ is the period of the sine function. This produces a square wave pulse with a 50\% duty cycle because a sine function spends $50 \%$ of the time above 0 and $50 \%$ below.

(a)

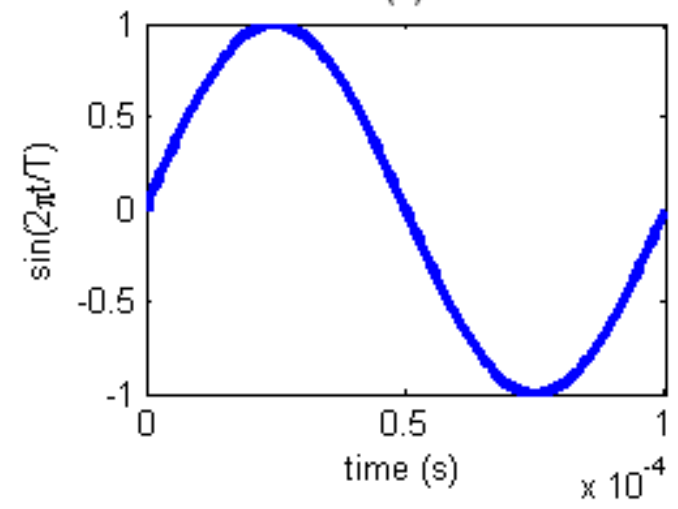

(c)

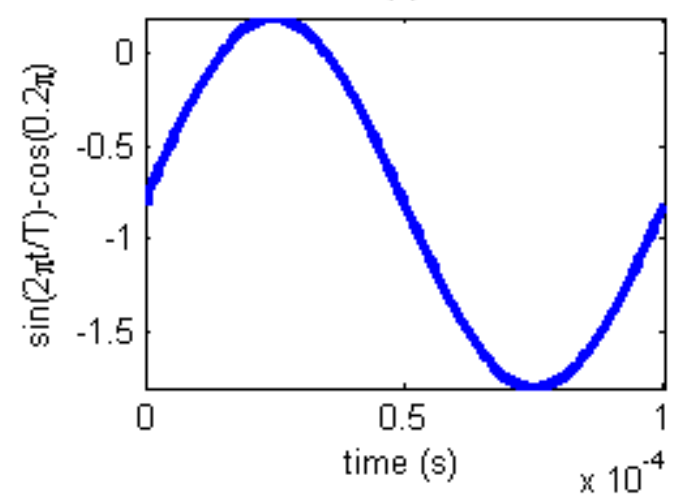

(b)

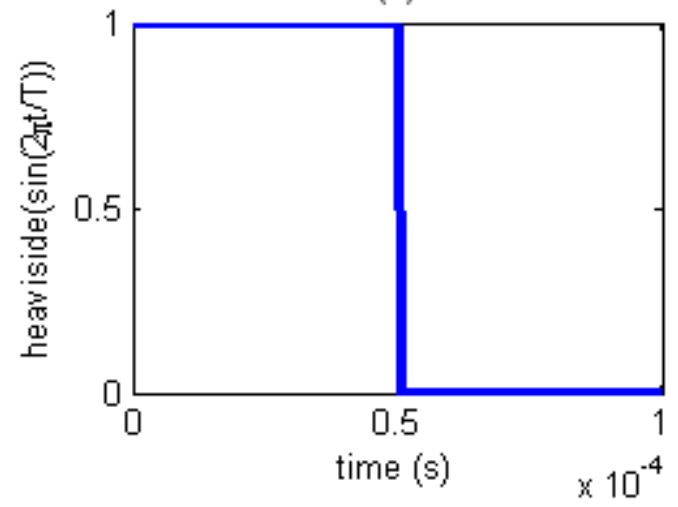

(d)

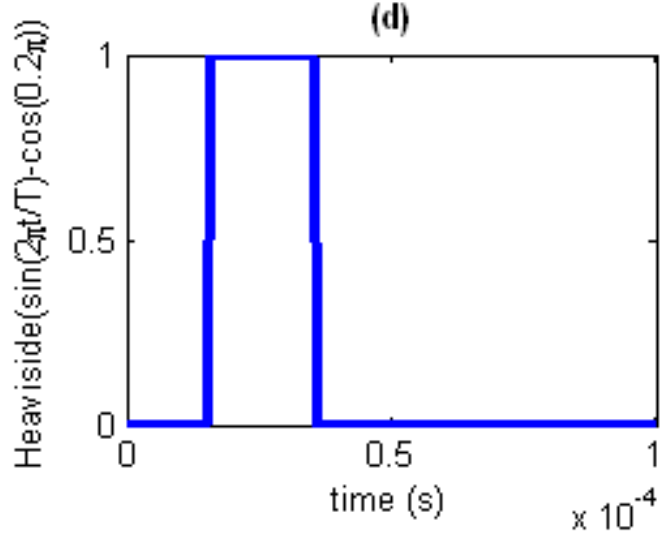

Figure 4: Generation of a time dependent square wave. (a) sine function, (b) $50 \%$ duty cycle smoothed Heaviside function, (c) sine function offset by $\cos (0.2 \pi)=0.628$, (d) $20 \%$ duty cycle smoothed Heaviside function.

To model a square wave pulse with a different duty cycle, the sine function that the Heaviside function operates on must be shifted up or down by an offset so that it spends 
the desired duty cycle above zero. To determine the correct amount of offset, we simply take the value of a cosine at the desired pulse width. For example, if we want a $20 \%$ duty cycle we shift the sine function by $\cos (0.2 \pi)$ (Figure $4 \mathrm{c})$. This produces the following square wave pulse with a $20 \%$ duty cycle (Figure 4 d). Note that the pulse is centered around the $\pi / 2$ point. This algorithm can be repeated indefinitely to produce a timedependent pulse train. The algorithm used in the software combines the spatial configuration of the beam with the temporal configuration.

The other important parameter in the pump algorithm is the input power. Care must be taken in determining the power per pulse in a time-dependent algorithm. The software itself ensures that the correct amount of power is available to each time increment in the run. That is, if the power input is set to one watt and the minimum time increment is 1 $\mu \mathrm{s}$, then the software provides $1 \mu \mathrm{W}$ to each time increment. Once the pump algorithm acts, this power will either be put into the material or not, thus the peak power is always the same but the average power will depend on duty cycle. This is the analog of a chopped pump beam where a constant amount of CW pump power is chopped to achieve a quasi-CW modulated pump beam.

If the goal is to model a Q-switched pump the average power is generally the same (as long as the reciprocal of the pulse repetition frequency, 1/PRF, is less than the relaxation lifetime of the pumping laser) but the peak pump power depends on PRF. Of note in this case is the fact that most Q-switched pump lasers used to pump $\mathrm{Cr}^{2+}$ lasers have a pulse width of $100 \mathrm{~ns}$ or less which is much smaller than the smallest practical time increment in our modeling program. We have approximated Q-switched pumping in our model by keeping average power constant, using a constant low duty cycle, and varying PRF. 


\subsubsection{Minimum time increment}

The FemLab ${ }^{\circledR}$ time dependent solver attempts to find a solution at discrete time increments throughout the total time period. The time increment chosen by the user should strike a balance between model accuracy and solution time required. It should also fully resolve the pulses in the pump algorithm. Time increment variations of 10, 5, 2 and $1 \mu$ s were modeled for a 10-kHz PRF and 15\% duty cycle in order to find the optimal time increment setting. Figure 5, below, shows the results of the z-average temperature at the $\mathrm{x}-\mathrm{y}$ center point for the different time increments.

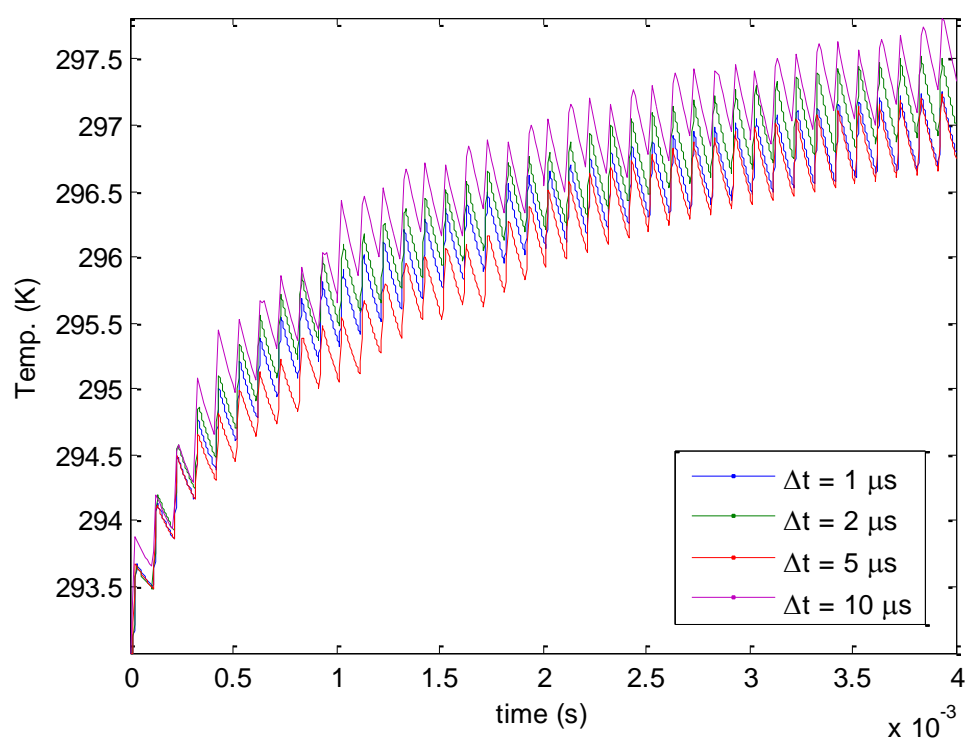

Figure 5: The z-average temperature at the $x-y$ center point for the different time increments over a total of $4 \mathrm{~ms}$

The difference between $1 \mu \mathrm{s}$ and $2 \mu \mathrm{s}$ is about $5.5 \%$ of the average temperature rise (above $293 \mathrm{~K}$ ). To study the differences between 1 and $2 \mu \mathrm{s}$, these models were run for 8 ms. 


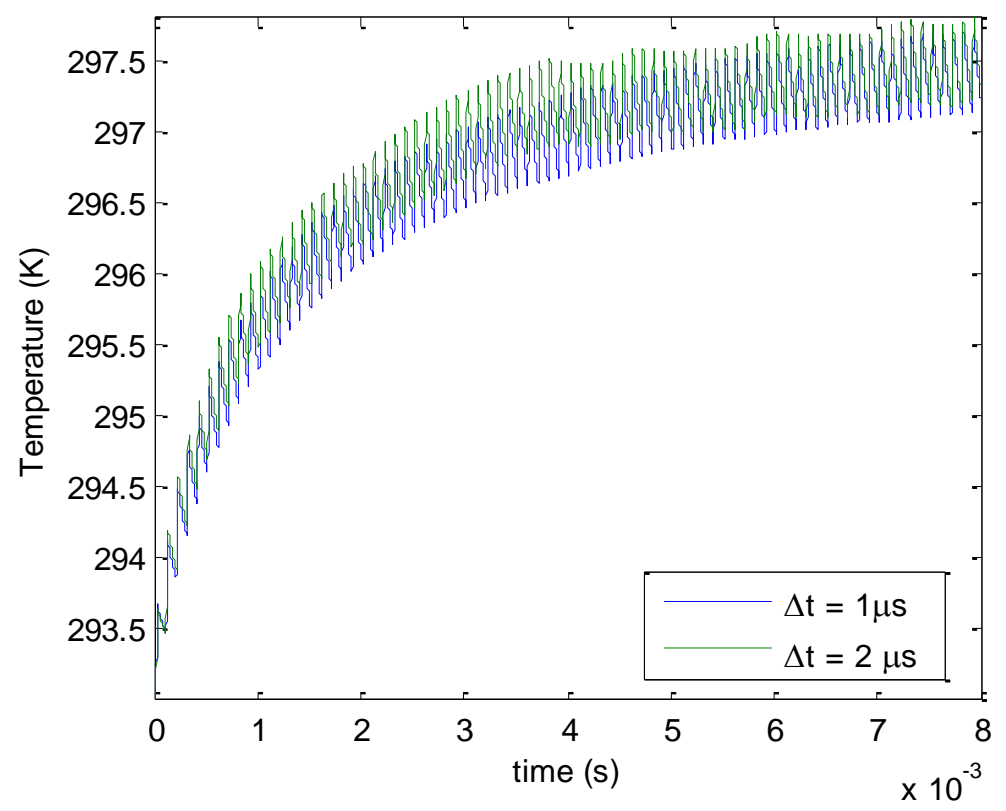

Figure 6: The z-average temperature at the $x-y$ center point comparison for two different time increments for a total of $8 \mathrm{~ms}$

As can be seen in Figure 6, the $1 \mu \mathrm{s}$ model seems to be the better model due to fluctuations in the $2 \mu \mathrm{s}$ model, although both are converging well at the end of $8 \mathrm{~ms}$, to within $2 \%$ of the average temperature rise. The smallest time increment feasible due to time and memory constraints was $1 \mu \mathrm{s}$. Modeling results for larger time increments were inconsistent throughout the model runs but converged on the $1 \mu$ s results. This indicated that $1 \mu \mathrm{s}$ was the optimal time increment to use for all models. This would indicate that the conductivity of $\mathrm{ZnSe}$ causes any changes in the heat equation to take about $1 \mu \mathrm{s}$ to propagate through the crystal enough to effect the finite element analysis. This could possibly change then by changing the number of elements (thus the size of each element).

Due to time and memory constraints, it is difficult to run a $1-\mu$ s time increment model for $8 \mathrm{~ms}$ or longer total time. It would be useful to be able to model longer total time periods by modeling shorter, discrete time periods. The last solution of the previous run would be used as the initial condition for the next model with the results merged into a 
continuous solution. The comparison, in Figure 7, of a continuous 8-ms model to two discrete 4-ms models showed that this method is acceptable as the differences in results between the two methods were negligible in comparison to the modeled values.

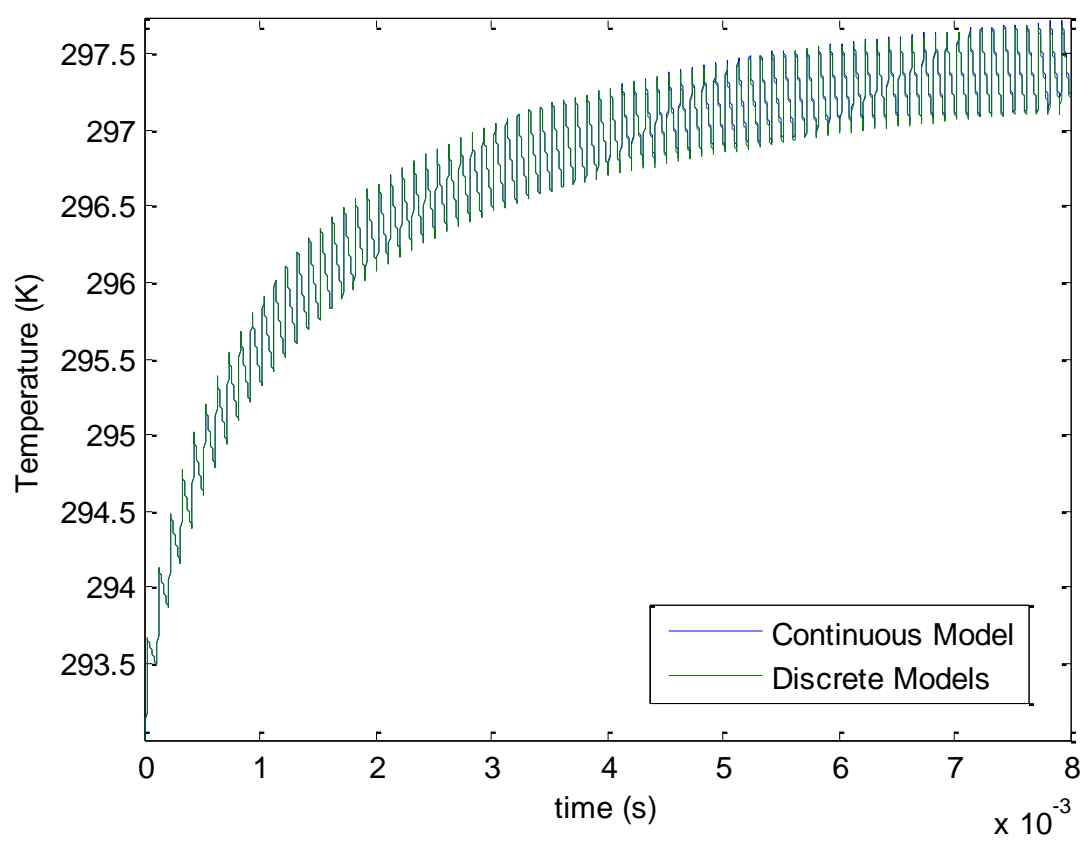

Figure 7: Comparison of single continuous model run to combine discrete runs showing acceptability of using discrete model runs for computational convenience

The $1-\mu \mathrm{s}$ time increment model was also run for $16 \mathrm{~ms}$ to see how closely the temperatures approached an asymptote. This data can be seen in Figure 8. 


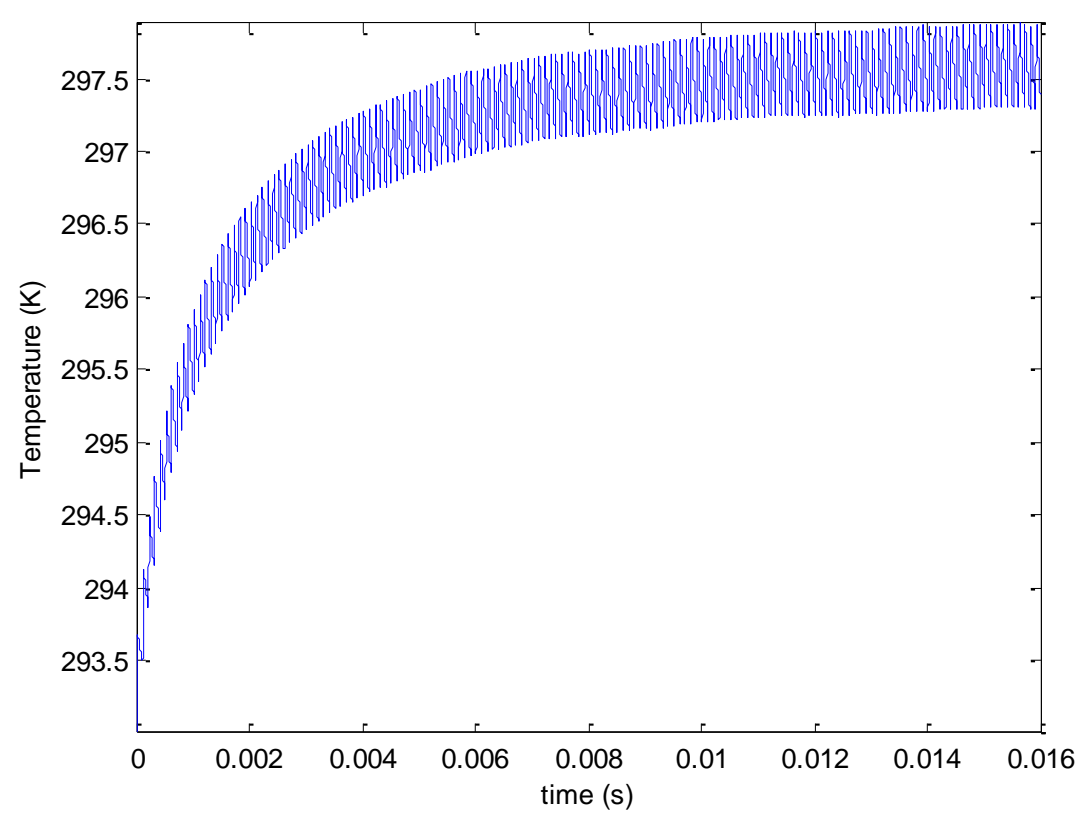

Figure 8: $16 \mathrm{~ms}$ modeling run at $1 \mu \mathrm{s}$ increments to determine asymptotic behavior

At $8 \mathrm{~ms}$, the z-average temperature rise (defined as the average temperature rise over a line through the depth of the sample in the propagation (z) direction) is within $0.2 \mathrm{~K}$ of the value at $16 \mathrm{~ms}$. This difference is less than $5 \%$ of the z-average temperature rise. This number is also within 5\% of the steady state average temperature rise of $9.3 \mathrm{~K}$. This confirmed that the maximum total time needed to run the models to achieve asymptotic behavior is $8 \mathrm{~ms}$.

\subsubsection{Determination of ancillary parameter values}

The goal of the time dependent modeling is to study the effects of different pulse repetition frequencies (PRFs) and duty cycle on both the magnitude of thermal lensing and the overall average temperature rise in the laser material. There are a large number of parameters in models such as this (geometry, time increment, mesh size, etc) and all of them except for PRF and duty cycle must be kept constant from run to run in order to 
make valid comparisons. A large portion of the modeling work involved determination of appropriate values for these ancillary parameters. The optimal value is one which produces accurate and precise results while still allowing for reasonable solution times.

In previous work, the maximum portion of the laser material geometry that needed to be included in the simulation was determined [2]. It was postulated that areas of the model farther from the center than approximately twice the pump beam radius would be irrelevant to the model solution and could thus be excluded. A set of basic face cooled models (modeled as parallelepipeds for simplicity) was constructed where the $\mathrm{x}$ and $\mathrm{y}$ dimensions varied from $2 \mathrm{~mm}$ to $8 \mathrm{~mm}$ while the pump beam $w$ (see eq. (3.1)) was 0.4 $\mathrm{mm}$. The thermal lensing power was found to not vary significantly in this range with size and 4-mm sides produced an upper bound on thermal lensing power and maximum average temperature rise. From these models, it was determined that the 4-mm $x$ and $y$ size was optimal.

Due to limited computer memory and CPU resources, it was desirable to run the models with as few degrees of freedom (a measure of the model complexity) as possible without sacrificing model quality. To insure that model complexity was not lowered to the point of affecting the results, the standard 4-mm model was run at varying degrees of freedom. The results showed that the thermal lensing power and maximum average temperature rise leveled off quickly and that as long as some reasonable mesh size was chosen, the results would be acceptable. With this software package, solution time was not the main impediment, memory consumption was. The most complex model that available 1 GB RAM computers could run was about 220000 degrees of freedom due to memory allocation issues. 
Once the appropriate $\mathrm{x}-\mathrm{y}$ dimensions and model mesh complexity were established, the geometry of the sample itself was studied. Due to symmetry properties, it was beneficial to use thin cylinders instead of parallelepipeds in the models. A 4-mm diameter cylinder was compared to the 4-mm by 4-mm parallelepiped to gauge any differences. Modeling showed that differences in thermal lensing power and maximum average temperature rise between the disk and parallelepiped were two orders of magnitude smaller than the lensing power itself. Thus the difference between the disk and parallelepiped geometries was negligible when looking at thermal lensing power and maximum average temperature rise.

\subsection{Model Results and Analysis}

Once the geometric models were constructed and configured as required, the finite element solver was initiated. FemLab® provided the solutions to the each time increment as data structures which contained all of the relevant temperature and geometry

data. It also provided a visual display of the solution in its post-processing mode. These solutions showed the temperature distribution along a slice along one axis through the origin. As an example, the solution for the 4-mm basic face cooled thin disk design can be seen in Figure 9 and Figure 10. 


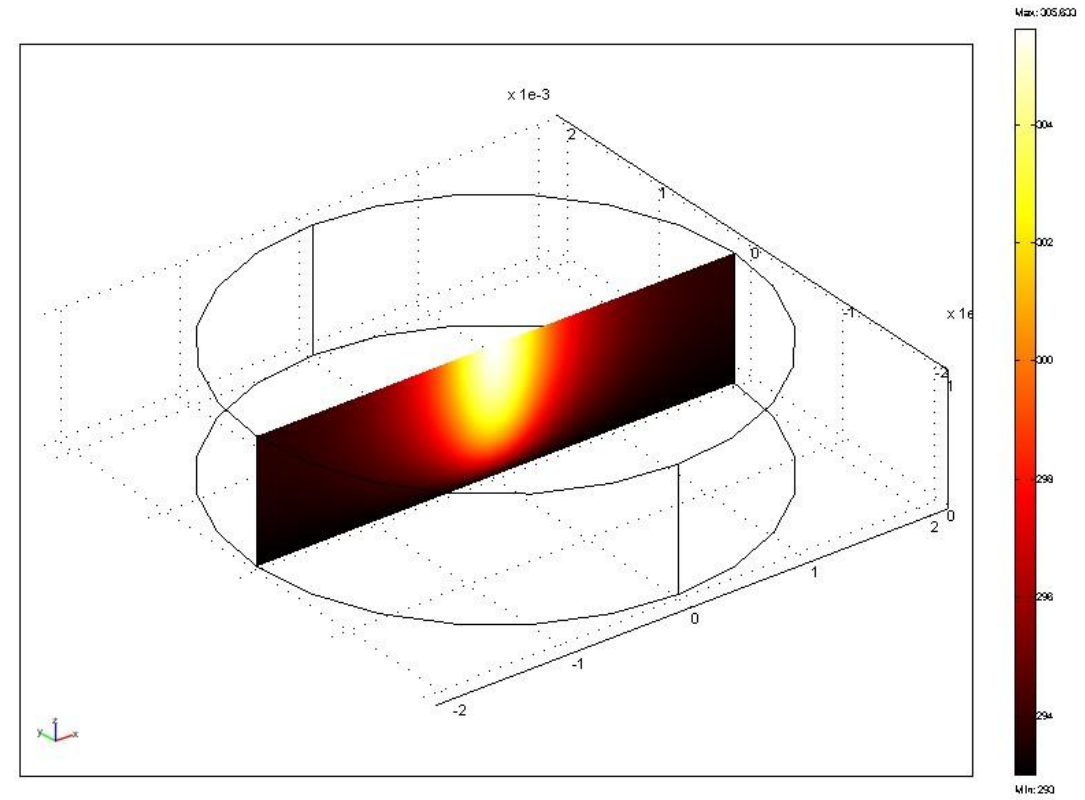

Figure 9: Simulation solution of $4 \mathrm{~mm}$ diameter, $1 \mathrm{~mm}$ thick face cooled thin disk pumped with a Gaussian $(n=1)$ beam with $\sigma=0.4 \mathrm{~mm}$. The temperature ranges from 293 (dark) to 306 (light).

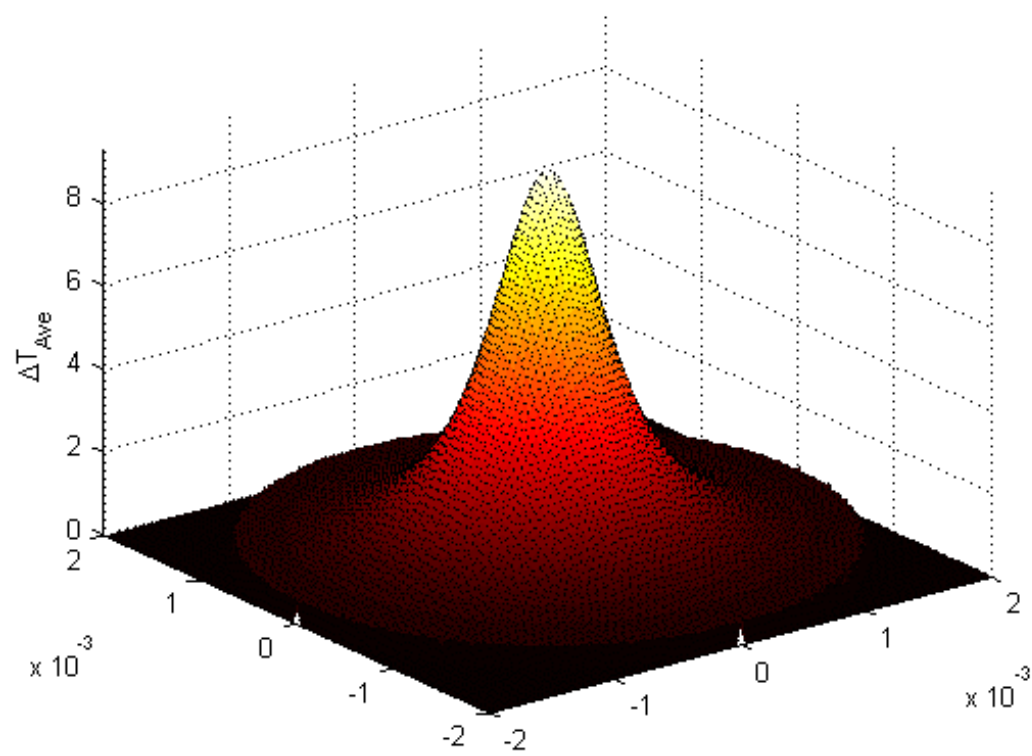

Figure 10: Temperature gradient surface for previous $4 \mathrm{~mm}$ face cooled thin disk solution

After a temperature solution for each mesh point was determined for every time increment, the raw temperature data needed to be processed into a more useful form. In this research, we were interested in two types of temperature information. The maximum 
$\mathrm{Z}$-average temperature rise in the laser material has an effect on the fluorescence lifetime while the radial gradient of the z-average temperature is an indication of the magnitude of the thermal lensing. To obtain the z-average temperature, the data for each time increment must be exported and then z-averaged. The radial temperature gradient is important because of the high thermo-optic coefficient of the laser material. As temperature increases, so does the refractive index which means that a temperature gradient will produce an effective quadratic thermal lens. The power of the thermal lens is related to how sharply the gradient peaks. The pump cycle has several interesting times at which the thermal gradient could be measured. These would be mid-way through the pulse, at the peak of the pulse, mid-way through the rest portion of the cycle and just prior to the pulse starting. Because lasing will start at the beginning of the pump pulse, that is where we measured the radial temperature gradient.

As mentioned in a previous section, most pulsed lasers fall into one of two categories. Lasers we will refer to as chopped lasers have a fixed peak power and an average power which varies with duty cycle, all of which are mostly independent of PRF. This would be analogous to directly modulating or physically chopping a CW fiber laser such as an IPG Tm:Fiber laser used in this research as a pump source. Lasers we will refer to as Qswitched lasers have a fixed average power, a fixed pulse width and a peak power and duty cycle which vary with PRF. This would be represented by the Tm:Ho:YLF laser used in previous works as a pump source for gain switched lasing of $\mathrm{Cr}^{2+}: \mathrm{ZnSe}$. A final category of laser which can be modeled is a hybrid system where the average power is fixed as in the Q-switched case, but the pulse width varies with the duty cycle as in the chopped case. Since no experimental analog to this was in use, it was not modeled. In 
our modeling calculations below we assumed $1 \mathrm{~W}$ of $\mathrm{cw}$ pump power; other powers can be considered by remembering that temperature rise and thermal lensing power scale linearly with pump power. We also assumed a 1 -mm thick disk $4 \mathrm{~mm}$ in diameter which absorbed all pump power.

\subsubsection{Chopped lasers}

In a chopped laser, the peak power is fixed, the average power is determined by the duty cycle and both are independent of the PRF. CW operation corresponds to $1 \mathrm{~W}$ peak and average power while $25 \%$ duty cycle, for example, is $1 \mathrm{~W}$ peak power and $0.25 \mathrm{~W}$ average power. For a $1-\mathrm{kHz}$ PRF at varying duty cycles, the $\mathrm{z}$-average temperature at the center point can be seen in Figure 11. Note that the temperature rise does not completely return to the baseline between pulses but the average temperature does reach an asymptotic value after about $8 \mathrm{~ms}$ of pumping. All duty cycles other than $\mathrm{cw}$ operation show a saw-tooth $(1-\exp (-t / \tau))$ rise and $(\exp (-t / \tau))$ decay at all times where $\tau$ is the relaxation lifetime. Also note that just $1 \mathrm{~W}$ cw can result in a $9 \mathrm{~K}$ temperature increase; multiple-watt pumping could easily reach temperatures where nonradiative relaxation would become severe. 


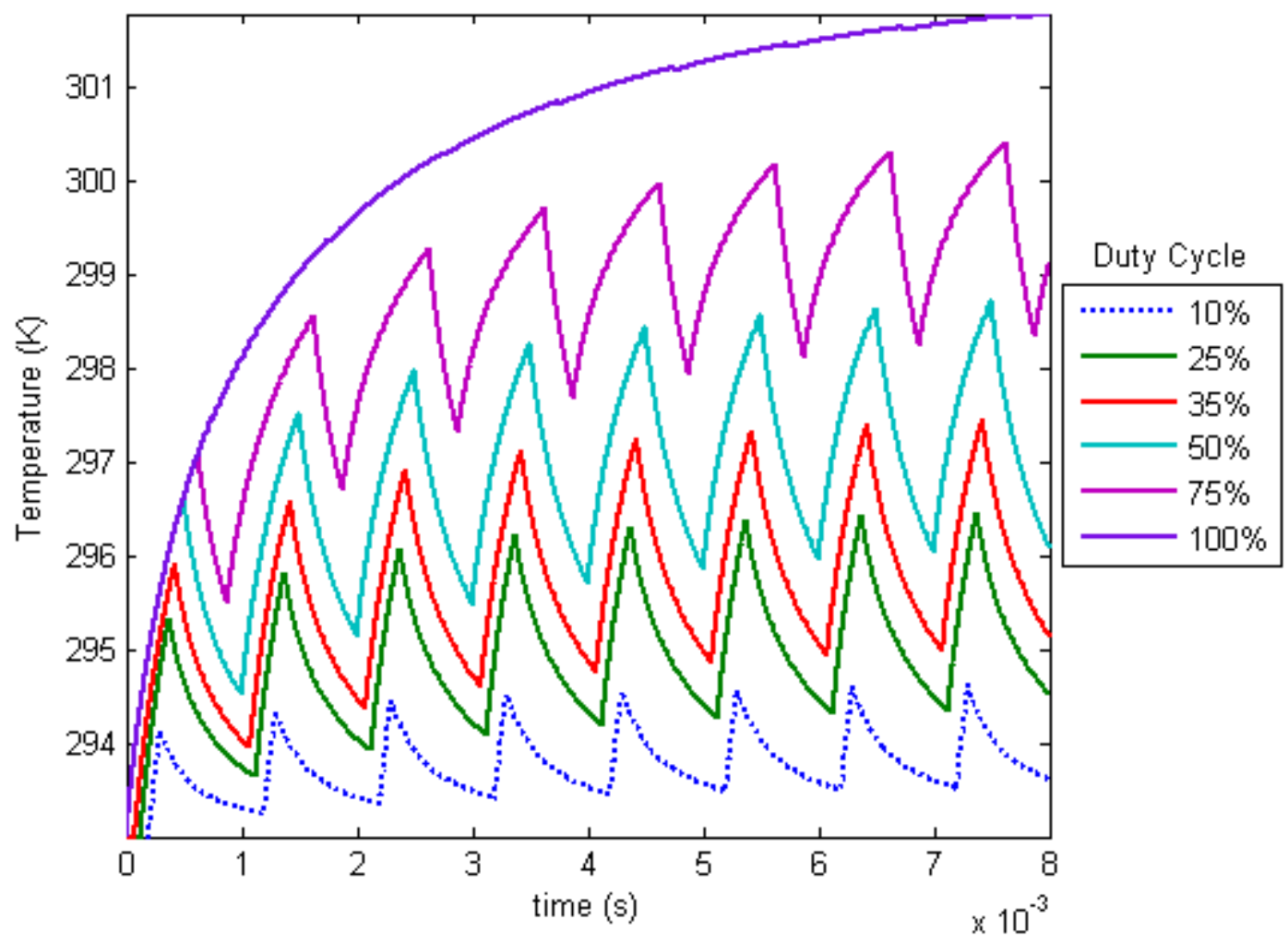

Figure 11: Center point z-average temperature for $1 \mathrm{kHz} P R F$ at varying duty cycles.

The radial thermal profiles for $1 \mathrm{kHz}$ PRF and varying duty cycles are shown in Figure 12. Table 3 shows calculations of thermal lensing power for the different duty cycles. Obviously, thermal lensing should increase with duty cycle (more average power); in fact, it increases quadratically with duty cycle due to increased pump power and decreased time to cool. 


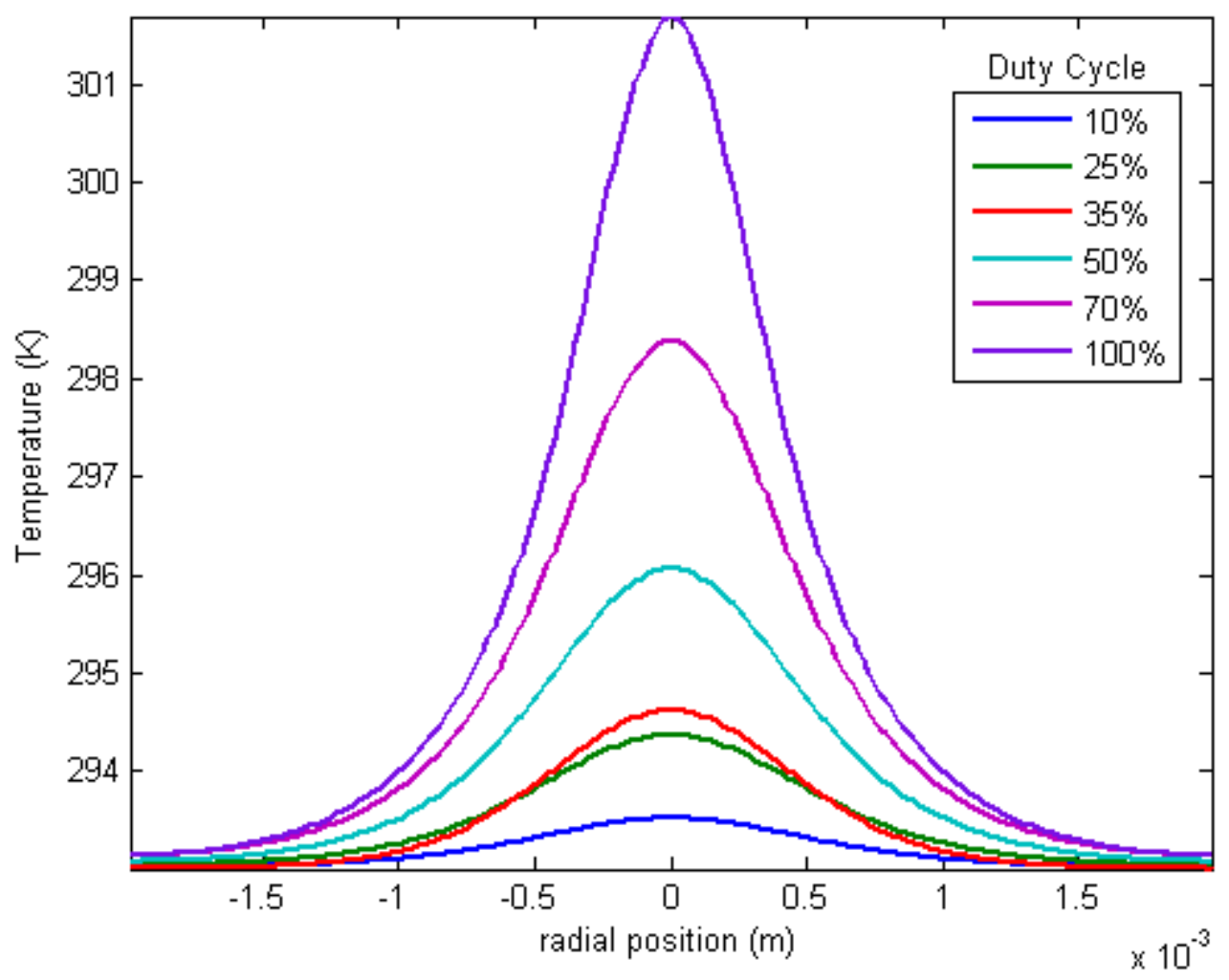

Figure 12: Radial temperature gradient for $1 \mathrm{kHz}$ PRF at varying duty cycles

Table 3: Thermal Lensing Power at $1 \mathrm{kHz}$ PRF, $1 \mathrm{~W}$ peak power, Chopped Laser

\begin{tabular}{|c|c|}
\hline Duty cycle $(\%)$ & Thermal lens power $\left(\mathrm{m}^{-1}\right)$ \\
\hline 10 & 0.23 \\
\hline 25 & 0.63 \\
\hline 35 & 0.88 \\
\hline 50 & 1.55 \\
\hline 75 & 3.06 \\
\hline 100 & 6.00 \\
\hline
\end{tabular}

These modeling results predict that lower duty cycles should better support lasing through lower temperatures and lower thermal lensing. This is supported experimentally as seen by the results in Figure 13 [37]. At low duty cycles the laser output power slope 
efficiency remained linear for all average pump powers up to 5 watts. However, the high duty cycle results showed rollover, i.e. reduced slope efficiency above $4.5 \mathrm{~W}$ average pump power for $50 \%$ duty cycle and above $3.7 \mathrm{~W}$ for $100 \%$ duty cycle operation. The threshold for rollover is not simply a function of average power because the pump-off times allow the laser material temperatures to cool below the $\mathrm{cw}$ average. The decrease in slope efficiency with increasing duty cycle is likely due to the increased average temperature of the sample thus reducing $\mathrm{Cr}^{2+}$ energy storage time. The onset of rollover is likely to be due to onset of resonator instability from thermal lensing.

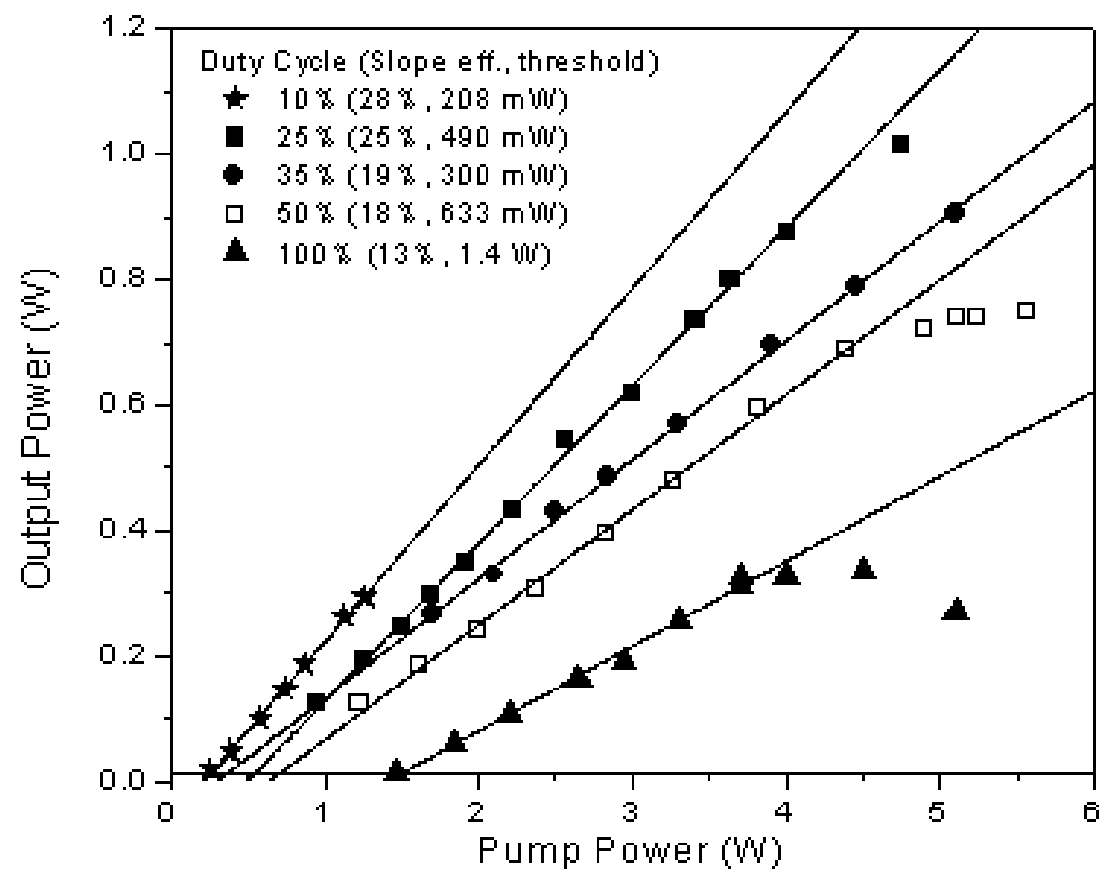

Figure 13: Experimental results for $1 \mathrm{kHz}$ PRF chopped laser with varying duty cycles. Pump power is average power. 


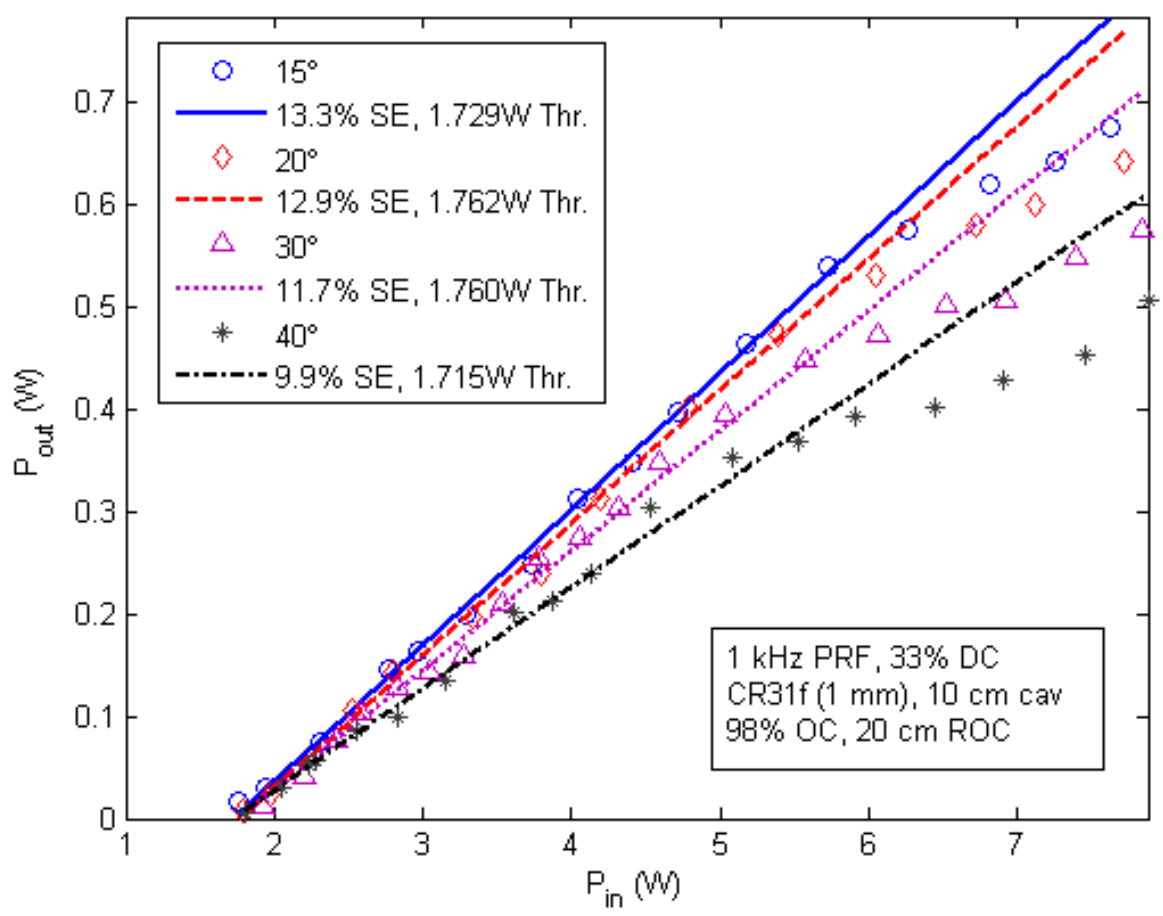

Figure 14: Experimental results for fixed PRF and duty cycles for varying heat sink temperatures

The model predicts that changing the heat sink temperature should only effect the slope efficiency and not the power drop-off associated with thermal lensing or the threshold power. In order to verify the influence of baseline heat sink temperature on the laser performance, a series of slope efficiency measurements were made at different temperatures. The other parameters were chosen based on previous measurements that indicated thermal lensing at higher powers. As Figure 14 indicates, the power drop-off at higher pump powers due to thermal lensing remains similar between the run as does the threshold power. The slope efficiency decreases as the heat sink temperature increases. Heat sink temperatures below $15^{\circ} \mathrm{C}$ caused condensation and were not suitable for laser use without dry air purging. 


\subsubsection{Q-switched Lasers}

In a system where the average power and duty cycle are fixed, variations in PRF have little effect on the overall time-average temperature at the center of the material. This is because, over a period of time which is large with respect to the pulse period, the same amount of heat is being put into the laser with the same relative amount of rest time (due to the fixed duty cycle). Figure 15 shows the z-average temperature for $15 \%$ duty cycle pumping and varying repetition rate. The model implicitly assumes instantaneous heating with absorption of pump power. It shows that during the pumping time (which is when gain induced $\mathrm{Cr}^{2+}$ lasing would be occurring), the average temperature goes higher as the repetition rate decreases. This contradicts the experimental observation that average $\mathrm{Cr}^{2+}$ output power is constant with increasing PRF until it approaches $10 \mathrm{kHz}$ and then output declines. However, pumped $\mathrm{Cr}^{2+}$ ions do not immediately transfer their stored energy to the crystal lattice; either stimulated emission or transition back to the ground state must occur. Thus the temperatures at the beginning of the pumping are likely to be more representative of the temperature during lasing for the Q-switched case. In that case, the minimum temperature increases with PRF reaching the cw limit as PRF goes to infinity.

The radial temperature gradients were extracted at the low temperature points of each PRF cycle in Figure 15 at times when the average temperature was close to steady state. This is based on the assumption that thermal lensing effects were most important at the beginning of the pump cycle. These gradients can be seen in Figure 16 and the calculated thermal lens powers associated with them are listed in Table 4. 


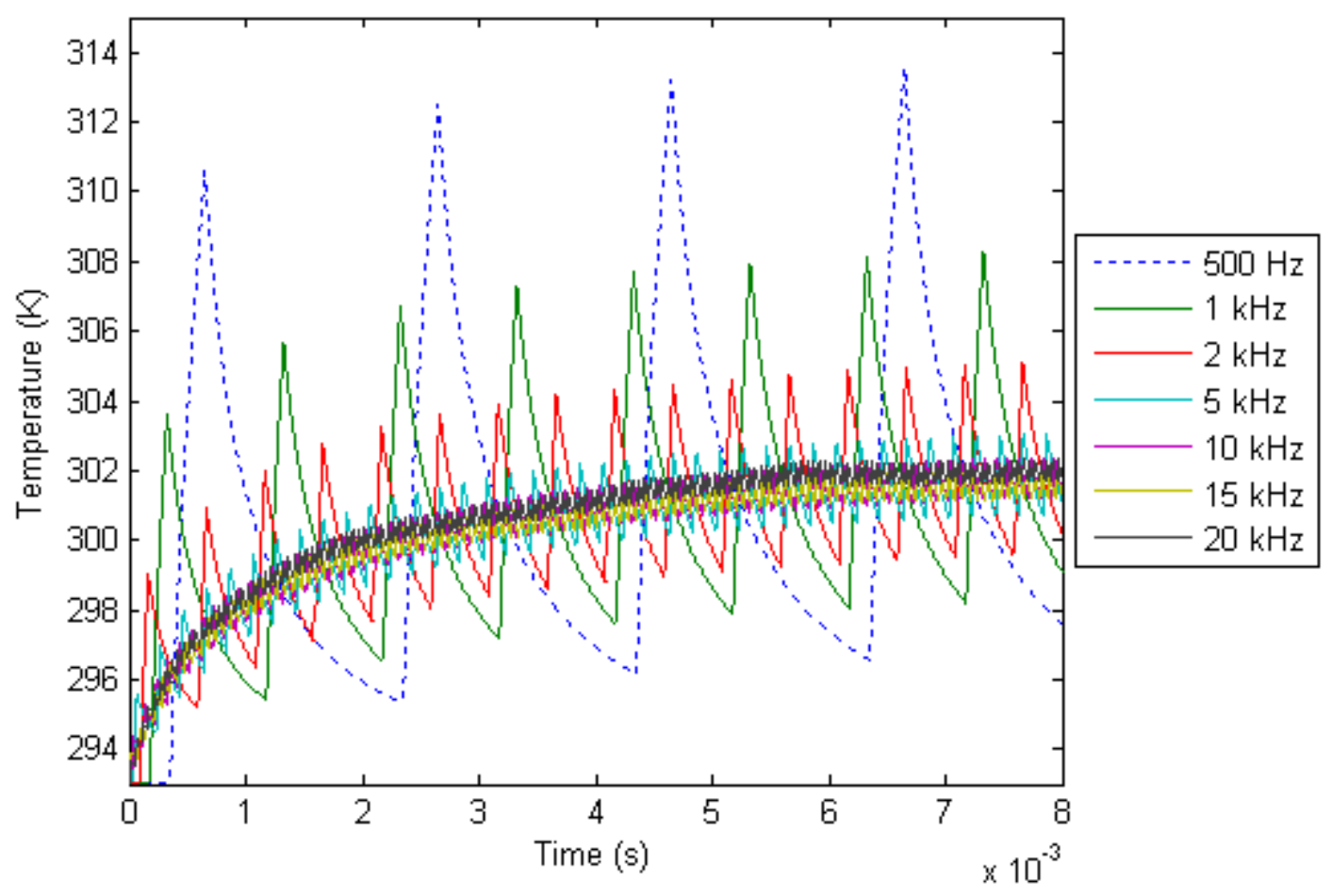

Figure 15: Center point $z$-average temperature for $15 \%$ duty cycle with varying PRF 


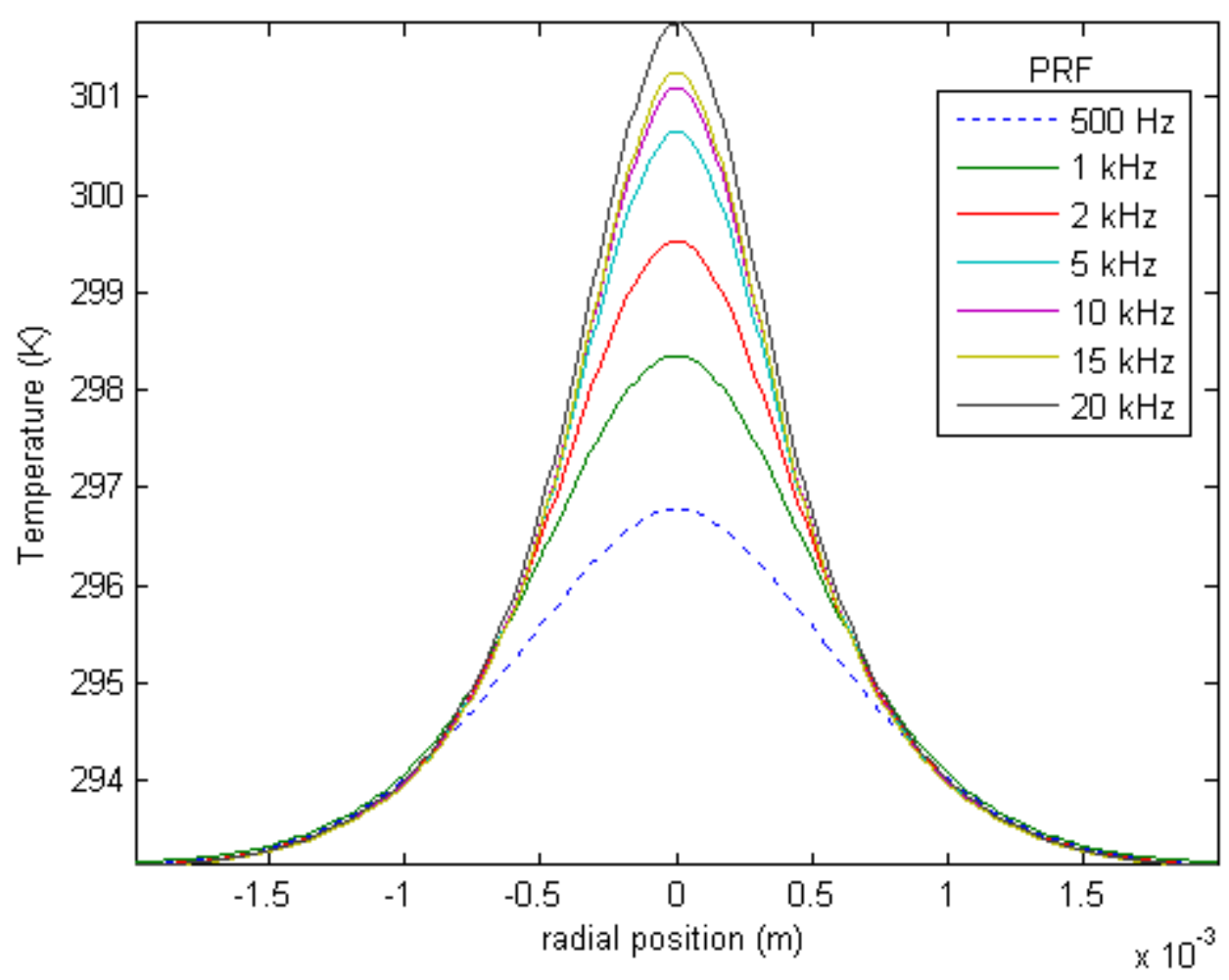

Figure 16: Radial temperature gradient for $15 \%$ duty cycle at varying PRFs.

Table 4: Thermal Lensing Power at $1 \mathrm{kHz}$ PRF, $1 \mathrm{~W}$ peak power, Q-switched Laser

\begin{tabular}{|c|c|}
\hline PRF $(\mathrm{kHz})$ & Thermal lens power $\left(\mathrm{m}^{-1}\right)$ \\
\hline 0.5 & 1.40 \\
\hline 1 & 2.43 \\
\hline 2 & 3.60 \\
\hline 5 & 4.84 \\
\hline 10 & 5.37 \\
\hline 15 & 5.59 \\
\hline 20 & 5.97 \\
\hline
\end{tabular}

\subsection{Modeling Conclusions}

Time-dependent modeling of thermal effects in laser materials is a viable option for understanding the influences of laser parameters such as PRF and duty cycle on laser 
performance. The model is in agreement with experimental results for the chopped pumping case but does not directly agree with Q-switched pumping because the time delay between absorption and heat transfer to the host material is not accounted for in our model. Future modeling work will focus on clarifications of the chopped laser results and on development of a way to model the time delay between Q-switched pumping and heating of the host material. 


\section{Chapter 4}

\section{Chromium-doped Zinc Selenide Fiber Laser}

\subsection{Introduction}

As previously mentioned, a number of military and scientific applications require laser sources in the mid-IR region. Due to the high stress environment in which these types of laser sources are likely to be used, they must be compact, rugged and solid-state. Vibration, dust, limited power and little to no cooling are all issues that must be addressed in any architecture of this type. Doped fiber amplifier laser systems are optimal candidates for this type of use due to their inherent properties. In addition to environmental ruggedization, multiple fibers could be used with a single pump source or vice-versa. This ability to possibly distribute laser power to various points around an aircraft body using a central pump source could be extremely useful in applications which require multiple output ports such as IRCM systems.

Silica fiber has long been used in the telecom industry as both a transport medium and for all-optical amplification applications such as Erbium Doped Fiber Amplifiers (EDFAs). An EDFA is essentially a normal optical fiber waveguide that has been lightly

doped with a rare-earth element, namely erbium (possibly co-doped with ytterbium to increase the pumping efficiency and gain). In a typical EDFA, $980 \mathrm{~nm}$ photons are used to create $1550 \mathrm{~nm}$ photons which serve to amplify signal photons at the same wavelength. 
Using this same idea, silica fibers are useful for the construction of fiber lasers. These glasses have a high damage threshold, their surface-to-volume ratio is high leading to easy heat dissipation, the gain medium is nicely contained in a waveguide and current fiber manufacturing capabilities make available a wide range of quality fibers. Due to the nature of silica (with strong absorption starting around $2 \mu \mathrm{m}$ ), silica fiber lasers have generally been limited to sub- $2 \mu \mathrm{m}$ applications. Commercially available lasers are commonly found using $\operatorname{Er}(1.5 \mu \mathrm{m})$ and $\operatorname{Tm}(1.9 \mu \mathrm{m})$.

Expanding on the available methods for thermal lensing mitigation, one goal was to demonstrate a chromium-doped zinc selenide $\left(\mathrm{Cr}^{2+}: \mathrm{ZnSe}\right)$ fiber amplifier. One method demonstrated in the literature for fabricating optical fibers from crystalline or polycrystalline laser hosts was to using a standard fiber drawing-tower method with a larger diameter, pre-fiber form or preform which was constructed using the rod-in-tube (RIT) method [49, 50]. Using this method, a silica rod was tapered at one end and had a hole drilled in the other. $\mathrm{A} \mathrm{Cr}^{2+}: \mathrm{ZnSe}$ crystal rod doped to the appropriate level was inserted in this hole, which was then capped with silica to contain the crystal. The dimensions of the preform and crystal were designed to give the desired core/cladding size ratio after drawing. The melting point of zinc selenide is $1526^{\circ} \mathrm{C}$ while the softening point of silica is $1670^{\circ} \mathrm{C}$ which should have allowed for consistent drawing. The coefficient of thermal expansion of zinc selenide is much higher than that of silica, so heating of the preform was done slowly to avoid thermal shock. Further, it was hoped that the preform could be manufactured to produce modified fiber designs such as a dualcore design to allow pumping in the outer core with signal amplification and guiding in the inner core. 


\subsection{Experimental Results}

Discussions were initiated with researchers at Clemson University to fabricate a preform like the one described above. In addition, $\mathrm{Cr}^{2+}: \mathrm{ZnSe}$ with the proper dimensions to fit in the perform hole was also needed. Previous samples of chromium zinc selenide were purchased from a commercial vendor which had subsequently gone out of business, so a search for a new vendor was initiated. One of our other sources of $\mathrm{Cr}^{2+}: \mathrm{ZnSe}$ in the past was MIT/Lincoln Labs. Costs for resumption of chromium doping by this group was discussed and fell in the $\$ 65 \mathrm{~K}$ range which was considered too high for the risk level of this project. After discussions with Sergey Mirov's group at the University of Alabama Birmingham (UAB), it was decided that they could provide the types of samples needed.

Prior to the procurement of new $\mathrm{Cr}^{2+}: \mathrm{ZnSe}$, two uncoated, chromium-doped zinc selenide samples of dimensions $10 \times 9 \times 2 \mathrm{~mm}$ and $8 \times 10 \times 2 \mathrm{~mm}$ respectively were sent to Clemson for use in fiber pulling experiments. These samples were crushed up and used as core-filler for a fiber preform (see Figure 17). These were to be used in place of the rods in order to begin producing fiber for testing. These samples had volumes of 180 $\mathrm{mm}^{3}$ and $160 \mathrm{~mm}^{3}$ respectively. The planned $50 \mathrm{~mm}$ long x $5 \mathrm{~mm}$ diameter core rod would have a volume of about $982 \mathrm{~mm}^{3}$, considerably larger than the given samples. In order to get a fiber draw of an appreciable length and good quality, both samples were used for one draw. The goal was to get a $125 \mu \mathrm{m}$ diameter, $25 \mu \mathrm{m}$ core fiber. The fiber preform used was an F300 tube, $6 \mathrm{~mm}$ ID x $30 \mathrm{~mm}$ OD x $400 \mathrm{~mm}$ length. Over $500 \mathrm{~m}$ of $125 \mu \mathrm{m}$ diameter fiber was produced from the preform. This draw was done under vacuum to minimize air inclusion. 


\section{Vacuum sealed}

Figure 17: Fiber preform design

Initial analysis indicated that the draw was not successful as most of the $\mathrm{Cr}^{2+}: \mathrm{ZnSe}$ seemed to have volatilized and moved further up the preform as heat was applied during drawing. As this material reached a cooler preform zone, it crystallized as seen in Figure 18. No $\mathrm{Cr}^{2+}$ seems to be present in the draw-end.

Because of this, subsequent draws were re-designed to fill any open portion of the preform not occupied by laser host with a silica rod and seal it.

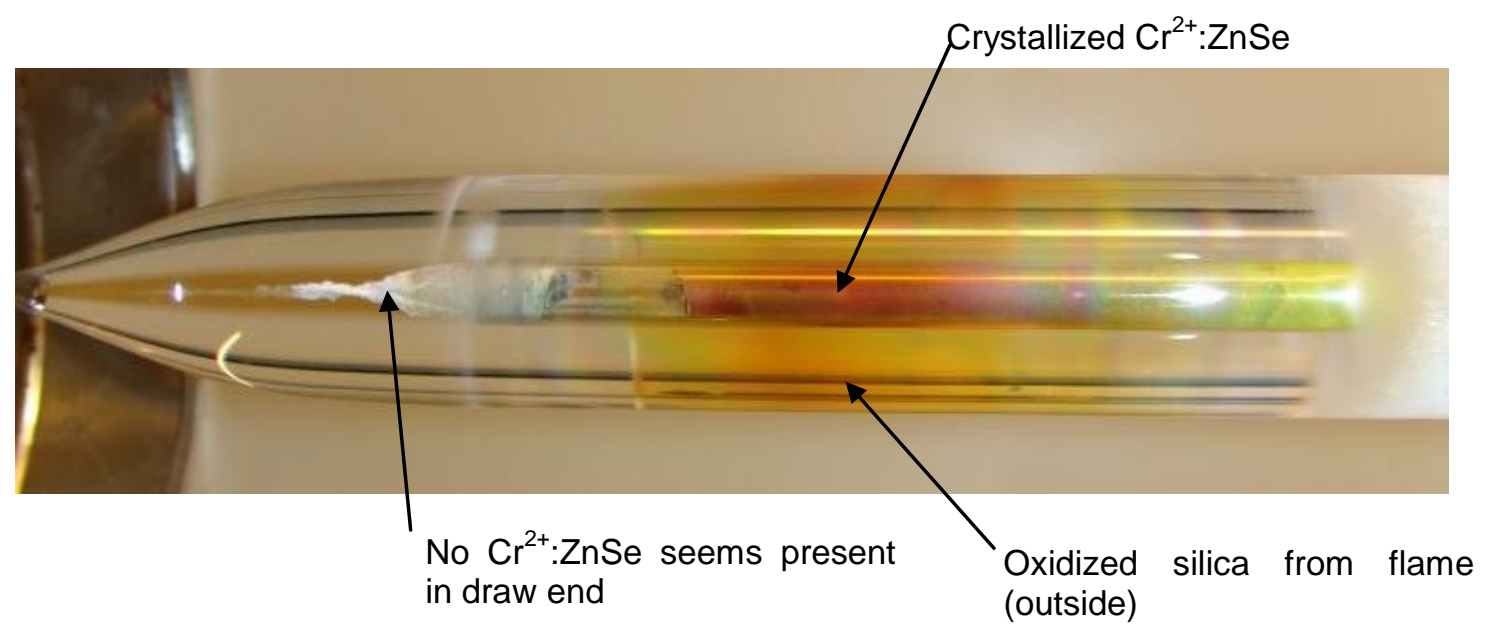

Figure 18: Preform remaining after first fiber draw attempt

Four of the six cylindrical ( $\sim \mathrm{mm}$ diameter by $48 \mathrm{~mm}$ length) $\mathrm{Cr}^{2+}: \mathrm{ZnSe}$ samples from UAB were received and were taken to Clemson University’s Center for Optical Materials Science and Engineering Technologies (COMSET) for fiber pulling. The second fiber draw attempt began with preform construction using COMSET's cutting edge lathe system. As seen in Figure 19, a Heraeus quartz tube, $30 \mathrm{~mm} \mathrm{OD,} 6 \mathrm{~mm}$ ID and about 
$300 \mathrm{~mm}$ long was first heated on one end while the ID was enlarged slightly using a carbon reamer. This was done to achieve a 'funnel' to facilitate the insertion of materials into the preform. This was then attached to a long, thin-walled hollow handle tube for the chuck (' $\mathbf{C}$ ') end. The purpose of this piece was to provide distance between the furnace and the chuck assembly in the drawing tower as well as to allow for connection to a vacuum manifold. A solid piece of quartz, $6 \mathrm{~mm}$ OD by $\sim 20 \mathrm{~mm}$ long was then inserted into the furnace ('F') end of the Heraeus tube and sealed by attaching a solid $20 \mathrm{~mm}$ OD by $\sim 200 \mathrm{~mm}$ long rod.

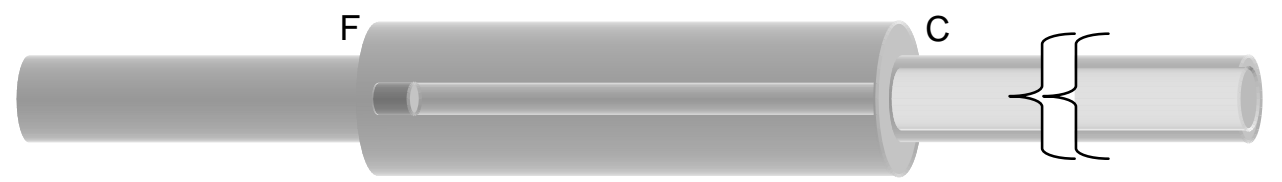

\section{Figure 19: Fiber preform assembly, step one}

The solid furnace-end rod was then melted near the tube and pulled to achieve a tapered end. The $\mathrm{Cr}^{2+}: \mathrm{ZnSe}$ cylinder was then slid down the hollow chuck end, through the funnel and down into place at the sealed furnace end. A $6 \mathrm{~mm}$ OD by $\sim 400 \mathrm{~mm}$ long 'tamper' tube was then inserted behind the $\mathrm{Cr}^{2+}: \mathrm{ZnSe}$ sample to reduce volatilization. The preform was evacuated and sealed at the very chuck-end of the hollow tube. The final preform can be seen in Figure 20. 


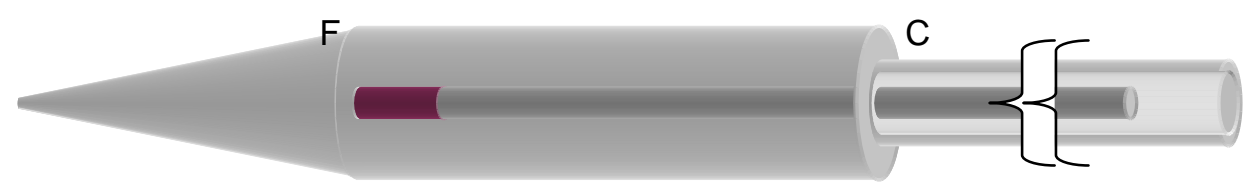

Figure 20: Preform construction, step two

The preform was lowered into the drawing tower furnace zone and a typical drawing routine was followed. The preform subsequently exploded while outside the draw zone which was at $1700^{\circ} \mathrm{C}$. The preform had 'light bulbed' or blown out into the furnace zone. The resulting damage to the preform can be seen in Figure 21. Due to the nature of zinc selenide, an emergency stop was performed and the room evacuated until the furnace completely cooled. An entire day of cleanup as well as furnace element and heat shield replacement followed. Zinc selenide begins sublimating at temperatures below the "drop point" (point at which the fiber can start being pulled) [51] and that this is the source of the pressure which caused the explosion. Further fiber draws were placed on hold until a safer draw method could be devised. 


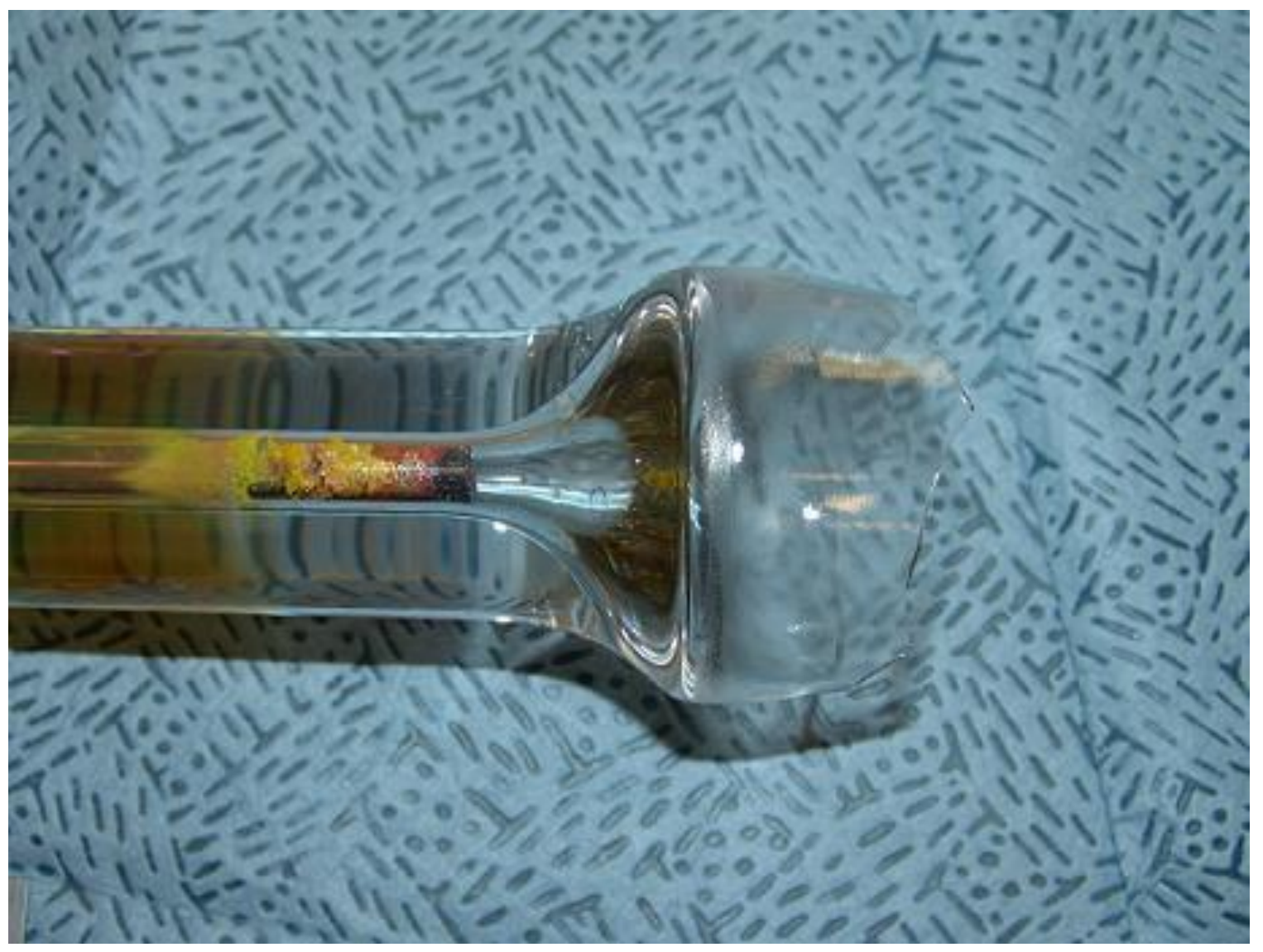

Figure 21: Exploded preform from second fiber draw attempt

Fluorescence decay lifetime measurements were made on the first fiber preform to look for signs of $\mathrm{Cr}^{2+}$ ions. In order to access the desired areas for analysis, the preform was roughly polished to remove oxidized silica and cleaved near the crystalline material, as seen in Figure 22. The $\sim 15 \mathrm{~mJ}$ excitation pulses, generated by a $1 \mathrm{~Hz}$, flashlamppumped, AO Q-switched Tm:Ho:Cr:YAG $2.1 \mu \mathrm{m}$ laser, were aimed at both the crystalline material location as well as a clear quartz location for a control. The lifetime results can be seen in Figure 23. The pulse shapes, as seen by an extended range InGaAs PIN diode showed that the core had a noticeable decay after the pulse when compared to 
the control. Using a MatLab curve fitting algorithm, the lifetime of the core was found to be $3.8 \mu \mathrm{s}$, which is very much in line with the measured lifetime of $\mathrm{Cr}^{2+}$ in other samples from the same doping run. This indicated a high likelihood that $\mathrm{Cr}^{2+}: \mathrm{ZnSe}$ can melt and re-crystallize while keeping the chromium in the important $2+$ valence state required for lasing in the 2-3 $\mu \mathrm{m}$ region.

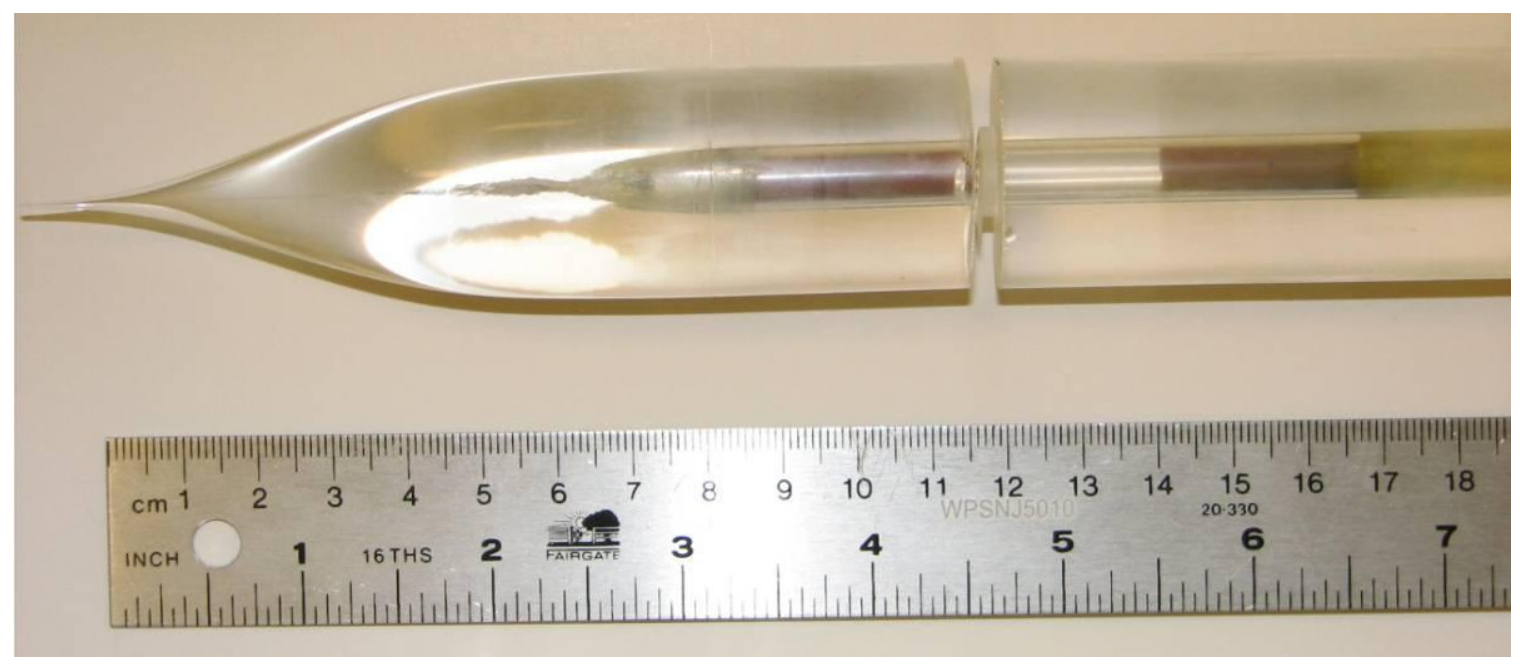

Figure 22: Polished and cleaved preform, ready for spectroscopy 

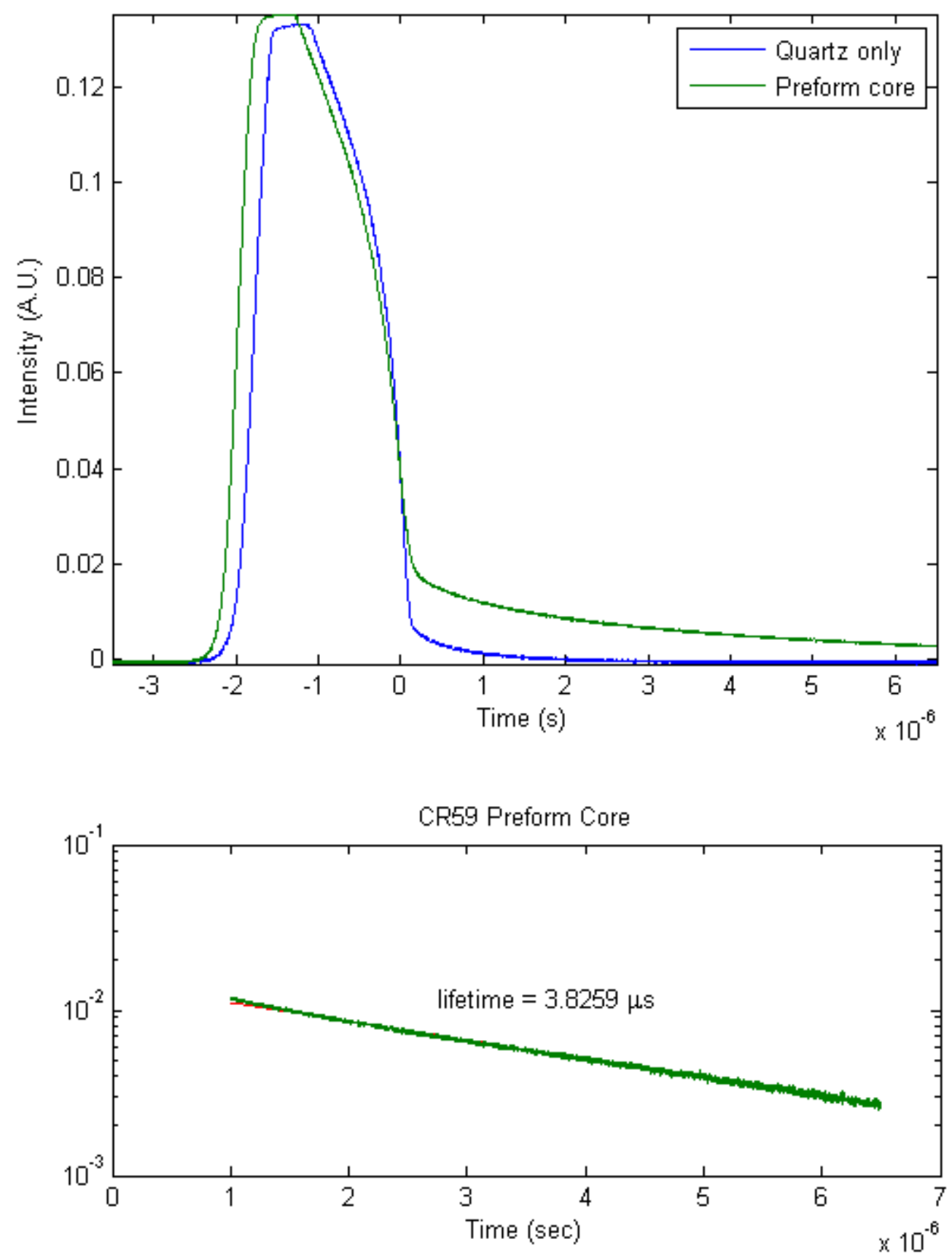

Figure 23a: Decay pulses from preform; 7b: Lifetime measurement of core

Spectroscopy was performed on the first fiber preform using an Acton SpectraPro 150 monochromator, SpectraHub detector interface, an extended range InGaAs detector and SpectraSense software. The results, seen in Figure 24, show definitively that the material 
which melted and re-solidified is indeed $\mathrm{Cr}^{2+}: \mathrm{ZnSe}$. The response of a mix of core and cladding show the expected strong absorption of the silica in the range above $2200 \mathrm{~nm}$.

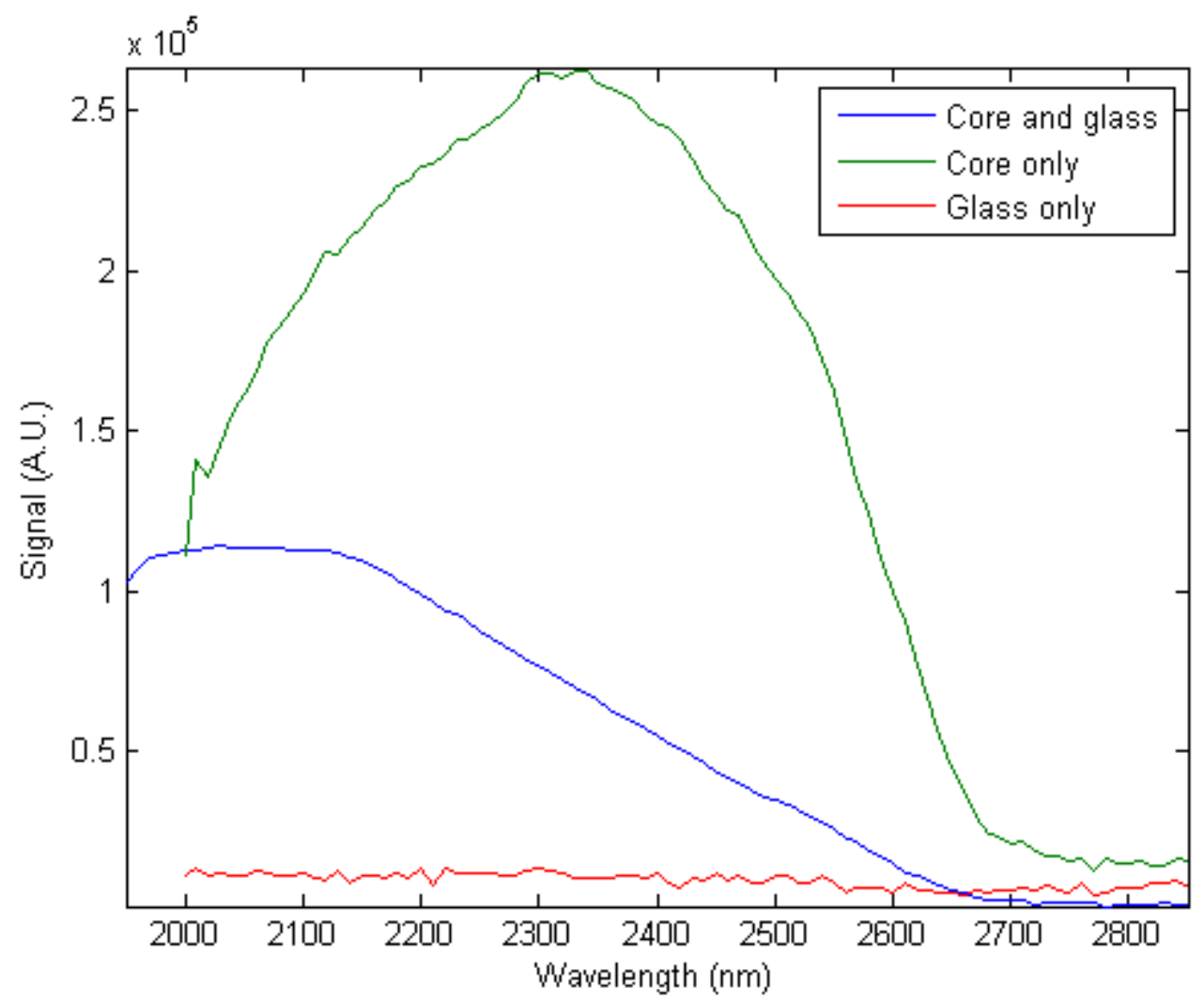

Figure 24: Spectroscopy results for different postions on fiber preform

A spectroscopy system, seen in Figure 25, for use with the experimental chromium fibers was assembled. The system was tested for calibration at $1900 \mathrm{~nm}$ using an IPG thulium fiber laser and three older samples of $\mathrm{Cr}^{2+}: \mathrm{ZnSe}$. The pump beam was chopped with a SciTech optical chopper running at $76 \mathrm{~Hz}$ and the detector, an Infrared Associates InSb, was read using a Stanford Research Systems SR830 lock-in amplifier connected to the SpectraHub. The slit widths were set at $1.17 \mu \mathrm{m}$ while the pump power was less than $30 \mathrm{~mW}$. Data taken with this setup confirmed that no detectable $\mathrm{Cr}^{2+}: \mathrm{ZnSe}$ was contained in the fiber drawn from the first preform. This confirms the speculation above 
that any $\mathrm{Cr}^{2+}: \mathrm{ZnSe}$ was vaporized and moved up and out of the draw zone before it could be incorporated into the fiber core.

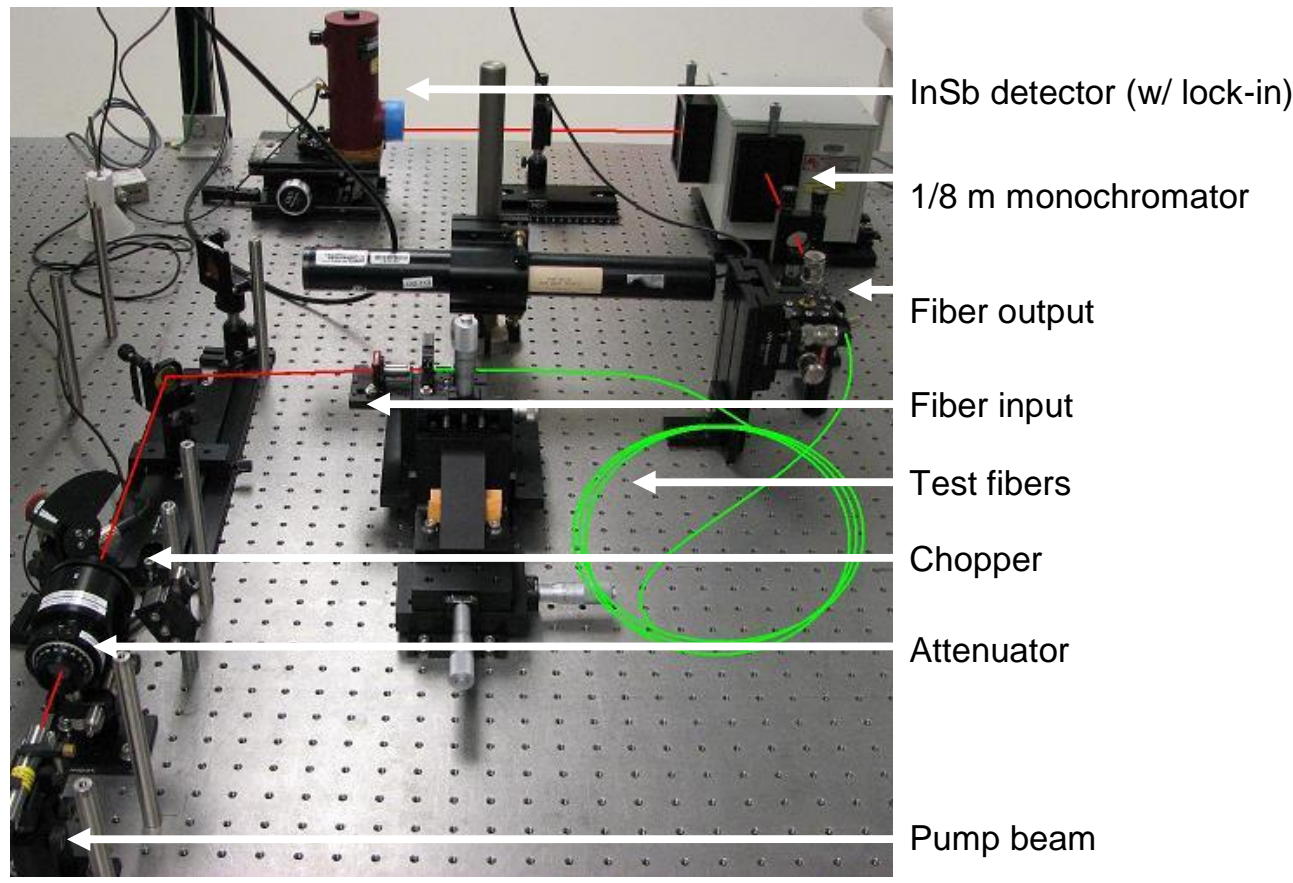

Figure 25: Fiber spectroscopy setup

COMSET proposed a new fiber preform construction and drawing experiment based on technical discussions of the volatilization problem. Two new preforms were constructed, one using a much shorter solid $\mathrm{Cr}^{2+}: \mathrm{ZnSe}$ cylinder (20 mm or less) and the other using a smaller diameter of crushed $\mathrm{Cr}^{2+}: \mathrm{ZnSe}$, as seen in Figure 26. Using a shorter cylinder should allow the preform to have all of the $\mathrm{ZnSe}$ in the hot zone of the furnace at the same time thus preventing sublimation and re-crystallization at another location along the $\mathrm{ZnSe}$ rod. The re-crystallization was to seal the vapor into a small volume. Reducing the volume of $\mathrm{ZnSe}$ being heated should also reduce the vapor pressure. The crushed, small diameter sample was to have low vapor pressure due to low volume and the crushed nature of the material should allow vapor to escape the hot zone. 

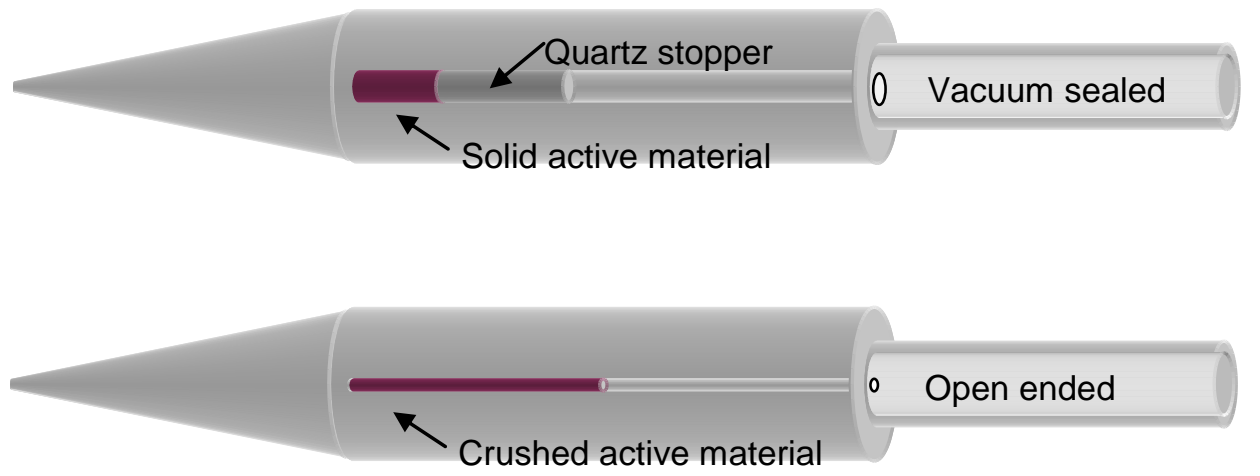

Figure 26: New preform designs a) solid, short core and b) long, small diameter powdered core

New fiber draws incorporating the new designs were attempted. As with all previous attempts save the first one, the preform exploded prior to the drop point.

\subsection{Conclusions}

Further fiber draws of $\mathrm{Cr}^{2+}: \mathrm{ZnSe}$ cores were not attempted due to the cost and hazards associated with preform explosions. It was hypothesized that the first draw only worked because the small amount of chromium zinc selenide powder in the first preform was completely volatilized up onto the walls of the upper cold section prior to drop, leaving no material to explode and resulting in no $\mathrm{Cr}^{2+}: \mathrm{ZnSe}$ incorporation into the fiber. Future work should shift to other techniques. Penn State University has shown promising developments in depositing metals and semiconductors in hollow core fibers [52]. It may also be possible to fabricate waveguide structures in bulk $\mathrm{Cr}^{2+}: \mathrm{ZnSe}$ using ultrashort laser pulses [53]. 


\section{Chapter 5}

\section{High-power, Widely-tunable $\mathrm{Cr}^{2+}: \mathrm{ZnSe}$ Lasers}

\subsection{Introduction}

This chapter presents work undertaken to implement lessons learned through modeling and previous work $[2,3,33,37,47,54]$ with face-cooled $\mathrm{Cr}^{2+}: \mathrm{ZnSe}$ lasers to construct high-power, widely-tunable laser systems. The previous work illustrated that the thermal issues limiting $\mathrm{Cr}^{2+}: \mathrm{ZnSe}$ laser performance can be addressed through appropriate choices of crystal geometry (helping to reduce thermal gradients), a longer wavelength pump source (reducing quantum-defect) and improved heat removal; all of which help to reduce the thermal load in the gain element. However, even when these steps are taken to mitigate the thermal problems, the lasers are often still limited in their output characteristics. One solution to this problem is to use a master oscillator power amplifier (MOPA) architecture where the MO design is chosen to emphasize a desirable operating characteristic such as beam quality, linewidth or tunability while the amplifier section accomplishes the power scaling without degrading performance.

This research investigated MOPA laser performance using polycrystalline $\mathrm{Cr}^{2+}: \mathrm{ZnSe}$ gain media in the conventional slab geometry for both the oscillator and the amplifier. We developed an improved amplifier gain model which incorporates variations in pump and signal beam parameters. We investigated a variety of MO configurations and their 
performance as part of a versatile MOPA-configuration laser system. We demonstrated greater than $10 \mathrm{~W}$ of pure continuous wave $(\mathrm{CW})$ output and compared the system performance to the theoretical model. System versatility was demonstrated by multiwatt, narrow linewidth output tunable over a range greater than $400 \mathrm{~nm}$. Beam quality and spectral content were also investigated.

\subsection{Amplifier Model}

In order to design a MOPA $\mathrm{Cr}^{2+}: \mathrm{ZnSe}$ laser, a theoretical model was developed and used to determine possible amplifier performance for a given set of physical parameters. We considered optical pumping co-propagating and co-aligned with an input signal beam. Absorption by the $\mathrm{Cr}^{2+}$ ions is proportional to pump intensity so both pump intensity and absorbed power will decrease along the propagation direction. In addition, both the pump and signal are expected to have some non-uniform transverse distribution which we will model as well.

Any amplifier model must be based on known or measured material parameters involved in absorption and emission. The absorption and emission cross-sections for $\mathrm{Cr}^{2+}: \mathrm{ZnSe}$ vary in the literature (for example, between $90 \times 10^{-18}$ and $130 \times 10^{-18} \mathrm{~cm}^{2}$ for peak emission cross-section). This model will use values published by DeLoach et. al. [27] but also acceptable would be those from Sorokina et. al. [46]. The absorption and emission cross-sections at the pump wavelength of $1.9 \mu \mathrm{m}, \sigma_{p a}$ and $\sigma_{p e}$ respectively, are $0.6 \times 10^{-18} \mathrm{~cm}^{2}$ and $0.3 \times 10^{-18} \mathrm{~cm}^{2}$ while the emission cross-section at the input signal wavelength of $2.5 \mu \mathrm{m}, \sigma_{s e}$, is $0.9 \times 10^{-18} \mathrm{~cm}^{2}$. The $\mathrm{Cr}^{2+}$ doping level, $n_{o}$, is calculated from the measured amount of a small signal (i.e. not close to saturating or bleaching the 
absorption) beam power transmitted through a $\mathrm{Cr}^{2+}: \mathrm{ZnSe}$ sample. A typical value of $n_{0}$ is $1 \times 10^{19} \mathrm{~cm}^{-3}$.

The electron configuration for a chromium ion is $[\mathrm{Ar}] 3 \mathrm{~d}^{5} 4 \mathrm{~s}^{1}$ and when doped into a IIVI material like $\mathrm{ZnSe}$, it will replace the group II element, zinc, as $\mathrm{Cr}^{2+}$ [55-57]. The electrons in the partially filled d orbitals interact strongly with the host crystal lattice giving rise to the broad tunability of $\mathrm{Cr}^{2+}$. $\mathrm{The}^{2+}$ ion in a tetrahedral crystal field is a 4-level system with fast relaxation in the vibronic sublevels which means that $n_{o}$ is effectively the sum of $n^{*}$ and $n_{g}$, where $n^{*}$ is the upper state population and $n_{g}$ is the unexcited (ground state) population. The energy level diagram shown in Figure 27 shows the $\mathrm{Cr}^{2+}: \mathrm{ZnSe}$ levels relative to the band gap and where the ground state is ${ }^{5} \mathrm{~T}_{2}\left(n_{g}\right)$ and the excited state is ${ }^{5} \mathrm{E}\left(n^{*}\right)$.

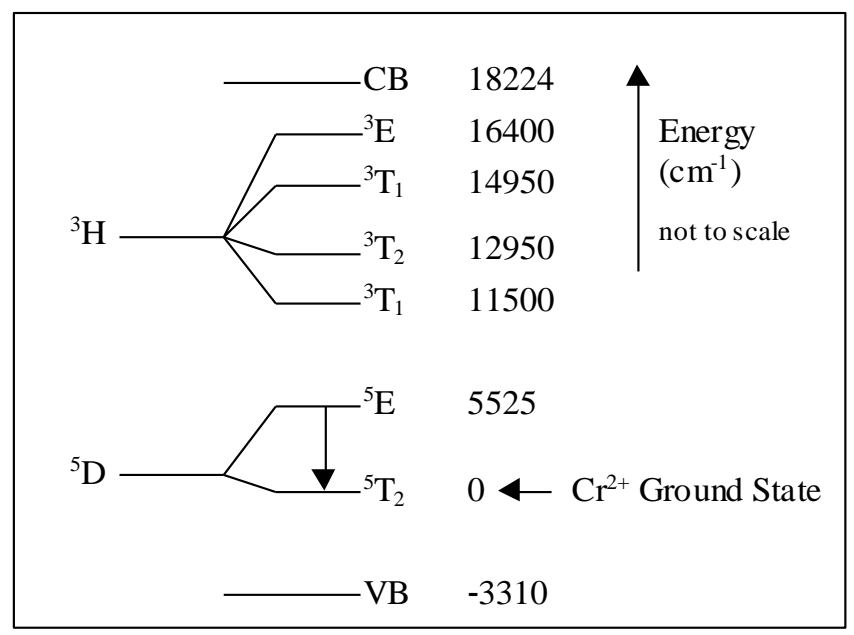

Figure 27: $\mathrm{Cr}^{2+}$ energy levels in $\mathrm{Cr}^{2+}: \mathrm{ZnSe}$ relative to the band gap [56]

In the case of a collinearly-pumped amplifier where both the pump and signal beams propagate in the $z$-direction, the intensity-driven $n^{*}$ will decrease with $z$ due to the same absorption which allows amplification. Thus the gain at each $z$ location will also vary due to the intensity dependence of the pumping rate. In order to calculate gain, $G$, in our 
amplifier we need to understand the relationships between $n^{*}$, the pump intensity, $I_{p}$ and the input signal intensity, $I_{s}$ through a system of differential equations.

The differential equation for $n^{*}$ will have a positive term from absorption at the pump wavelength and negative terms from spontaneous emission and stimulated emission at the signal wavelength. In addition to these, because the absorption and emission bands of $\mathrm{Cr}^{2+}: \mathrm{ZnSe}$ overlap in the $1.9 \mu \mathrm{m}$ region, there will also be a stimulated re-emission term at the pump wavelength as well. The absorption cross section at $2.5 \mu \mathrm{m}$ is negligible in $\mathrm{Cr}^{2+}: \mathrm{ZnSe}$ so there will be no absorption term at the signal wavelength. The differential equation for the pump radiation intensity will have negative terms from absorption and passive losses at the pump wavelength in addition to a positive term due to stimulated reemission at the pump wavelength. The differential equation for input signal intensity has a gain contribution from stimulated emission and passive losses at the signal wavelength.

This system can be described as

$$
\begin{gathered}
\frac{\partial n^{*}(t, z, r)}{\partial t}=\frac{I_{p}(t, z, r)}{h v_{p}}\left(\sigma_{p a} n_{g}(t, z, r)-\sigma_{p e} n^{*}(t, z, r)\right) \\
-\frac{I_{s}(t, z, r) \sigma_{s e}}{h v_{s}} n^{*}(t, z, r)-\frac{n^{*}(t, z, r)}{\tau} \\
\frac{\partial I_{p}(z, r)}{\partial z}=I_{p}(z, r)\left[-\sigma_{p a}\left(n_{0}-n^{*}(z, r)\right)+\sigma_{p e} n^{*}(z, r)-\gamma_{p, p a s s i v e}\right] \\
\frac{\partial I_{s}(z, r)}{\partial z}=I_{s}(z, r)\left(\sigma_{s e} n^{*}(z, r)-\gamma_{s, \text { passive }}\right)
\end{gathered}
$$

where $v_{p}$ and $v_{s}$ are the pump and signal frequencies and $\tau$ is the lifetime of the excited state. At equilibrium and with no other time-dependence, we can solve (5.1) to get 


$$
n^{*}(z, r)=\frac{\frac{I_{p}(z, r)}{h v_{p}} \sigma_{p a} n_{0}}{\frac{I_{p}(z, r)}{h v_{p}}\left(\sigma_{p a}+\sigma_{p e}\right)+\frac{I_{s}(z, r)}{h v_{s}} \sigma_{s e}+\frac{1}{\tau}}
$$

For a $\mathrm{Cr}^{2+}$ doping level of $n_{0}$, at room temperature $\tau$ is typically about $\sim 4 \mu \mathrm{s}$ [58]. It is interesting to note that, for a pump wavelength of $1.9 \mu \mathrm{m}$,

$$
\lim _{p}\left(n^{*}\right)=\frac{\sigma_{p a}}{\sigma_{p a}+\sigma_{p e}} n_{0}
$$

which in this case means that $n^{*}$ can reach a maximum of $2 / 3 n_{0}$.

It is convenient to define the pump and signal intensities in dimensionless units so that

$$
\begin{gathered}
J_{p}(z, r)=\frac{I_{p}(z, r)}{h v_{p}}\left(\sigma_{p a}+\sigma_{p e}\right) \tau=\frac{I_{p}(z, r)}{I_{p, s a t}} \\
J_{s}(z, r)=\frac{I_{s}(z, r)}{h v_{s}} \sigma_{s e} \tau=\frac{I_{s}(z, r)}{I_{s, s a t}}
\end{gathered}
$$

where $I_{s, s a t}$ and $I_{s, s a t}$ are the saturation intensities for signal and pump wavelengths. With these definitions, neglecting radial dependence and assuming negligible passive losses gives the system

$$
\begin{aligned}
n^{*}(z)= & n_{0} \frac{\sigma_{p a}}{\left(\sigma_{p a}+\sigma_{p e}\right)} \frac{J_{p}(z)}{J_{p}(z)+J_{s}(z)+1} \\
\frac{d J_{p}(z)}{d z}= & J_{p}(z)\left[-\sigma_{p a} n_{0}+\left(\sigma_{p a}+\sigma_{p e}\right) n^{*}(z)\right] \\
& \frac{d J_{s}(z)}{d z}=J_{s}(z) \sigma_{s e} n^{*}(z)
\end{aligned}
$$

These equations can be solved numerically for some initial pump and signal intensities, $I_{p 0}$ and $I_{s 0}$ and a crystal length $L$. This system assumes that both the signal and pump beams of a given power have a uniform transverse intensity over a circular area $A$ (with say a radius of $r$ ). In practice, however, the pump and signal beams both 
have some non-uniform, mostly Gaussian transverse intensity distribution. In order to model the radial dependence, we must run the $z$-propagation model for points along the radius with intensities given by the Gaussian distribution (5.11) of $r$ (with a $1 / \mathrm{e}^{2}$ radius of $\delta$ and a Gaussian order $N$ ) and integrate them over $r$ and $\theta$ to get an output power.

$$
I(r, \delta, N)=\exp \left(-\frac{2 r^{2 N}}{\delta^{2 N}}\right)
$$

This can take into account non-uniform saturation effects in the center of the signal beam where intensities are higher. The end result of which could be beams which become less Gaussian as they are amplified.

A working model was developed in MATLAB to represent our desired experimental configuration. Example model predictions of amplifier performance as a function of varying input signal levels and spot sizes can be seen Figure 28, below, for a crystal length of $7.8 \mathrm{~mm}$. The gain shows little sign of saturation effects due to pump or signal intensities until very high values $\left(\sim 10^{10} \mathrm{~W} / \mathrm{m}^{2}\right)$. The information gained from the model results was used in the next section to drive the experimental design of the MOPA system. From these models, the amplifier efficiency for a typical beam size of $40-80 \mu \mathrm{m}$ radius is around $50 \%$, which is similar to the types of efficiencies we see in high power laser oscillators. Therefore, as long as good extraction efficiency can be achieved experimentally, this approach is very promising for power scaling of $\mathrm{Cr}^{2+}: \mathrm{ZnSe}$ laser systems. 

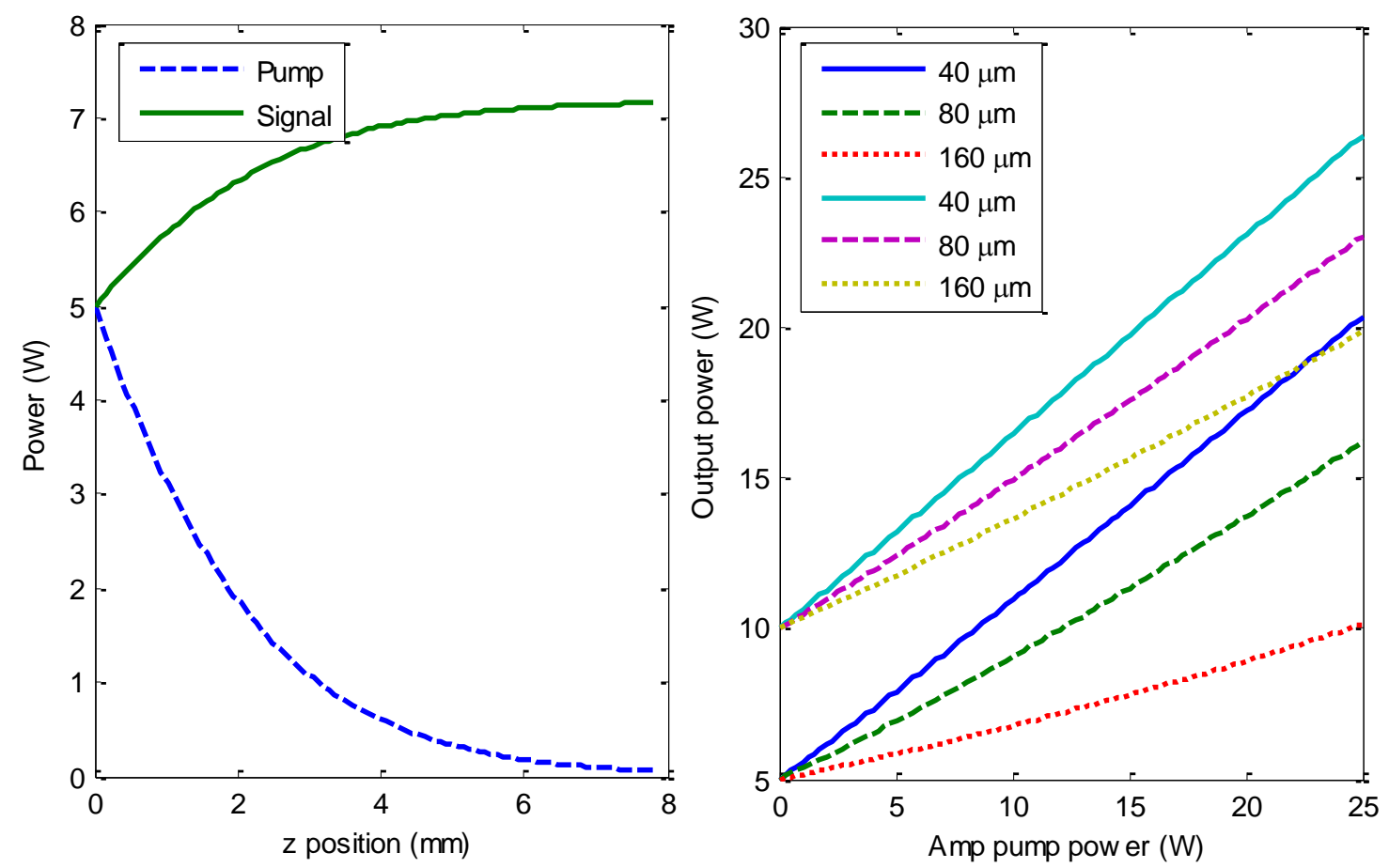

Figure 28: Example model predictions showing (left) input signal growth and pump absorption over the crystal length for $5 \mathrm{~W}$ input signal and $5 \mathrm{~W}$ pump, both at $80 \mu \mathrm{m}$ spot size and (right) amplification comparisons for 5 and $10 \mathrm{~W}$ input signal powers with varying spot size as a function of pump power.

\subsection{Experimental Design}

One of the advantages of the MOPA design is the flexibility to use almost any combination of oscillator and amplifier. A variety of MO designs were built and tested with the simplest PA design. This was done to evaluate the advantages of each design while adhering to the optimum PA design with respect to simplicity and performance predicted by the model. MO designs were selected for their ability to optimize certain operating modes such as tunability, narrow linewidth or power output.

There are a number of situations which may require the orientation of the laser crystal at Brewster's angle in a laser cavity. Anti-reflective (AR) coatings in the $2-3 \mu \mathrm{m}$ region may be too costly or not robust enough for use and thus another method is needed to reduce reflective losses. However, using a crystal at Brewster's angle carries with it 
some negative astigmatism effects which are compounded by thermal lensing in the crystal which is now at an angle. To counteract this, advantage can be taken of the astigmatism-compensating properties of cavity configurations first used by Kogelnik in dye laser cavities [59].

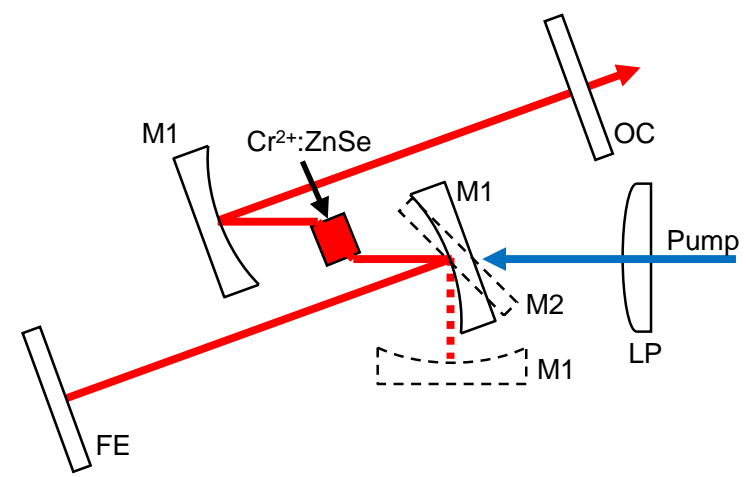

Figure 29: Astigmatically-compensated $Z$-cavity design which can use either a single collimated-leg design (dashed) or a double-collimated leg design. $\mathrm{OC}=$ output coupler, $\mathrm{LP}=$ laser pump lens, $\mathrm{FE}=$ functional element.

Our initial approach used a Kogelnik-type cavity with a single collimated leg represented in Figure 29 by the dashed lines. The cavity consisted of a flat $45^{\circ}$ dichroic input coupler (M2), AR-coated for $1.9 \mu \mathrm{m}$ and high reflectivity (HR)-coated for 2-3 $\mu \mathrm{m}$; two $5 \mathrm{~cm}$ radius of curvature (ROC) spherical mirrors (M1); and a flat $\mathrm{CaF}_{2} 50 \% \mathrm{R}$ output coupler (OC) located $\sim 16 \mathrm{~cm}$ from M1. The pump focusing lens (LP) had an experimentally-determined optimal focal length of $5 \mathrm{~cm}$. The polycrystalline, 8.9x7.0x3.0 $\mathrm{mm}(\mathrm{LxWxH}) \mathrm{Cr}^{2+}: \mathrm{ZnSe}$ gain element was pumped along the long dimension with a Tm-fiber laser linearly polarized parallel to the plane of the diagram. The fiber nature of the pump system and the lack of high-power isolators at $1.9 \mu \mathrm{m}$ required that this and all subsequent designs incorporate a non-normal incidence input coupler to prevent back-reflection. This cavity was shown to be capable of over $12 \mathrm{~W}$ of pure CW power [4] and better than $40 \%$ slope efficiency. This cavity was limited however, in that its output beam quality and stability were poor at higher operating 
powers which essentially precluded quality focusing and overlap in an amplifier. It also had no good location in which to insert functional elements such as a grating for tunability or a saturable absorber for modelocking. As can be seen in Figure 30, this cavity has periods of instability in its output (denoted by larger error bars). This was postulated to be due to the cavity changing modes to compensate for increasing thermal load in the system.

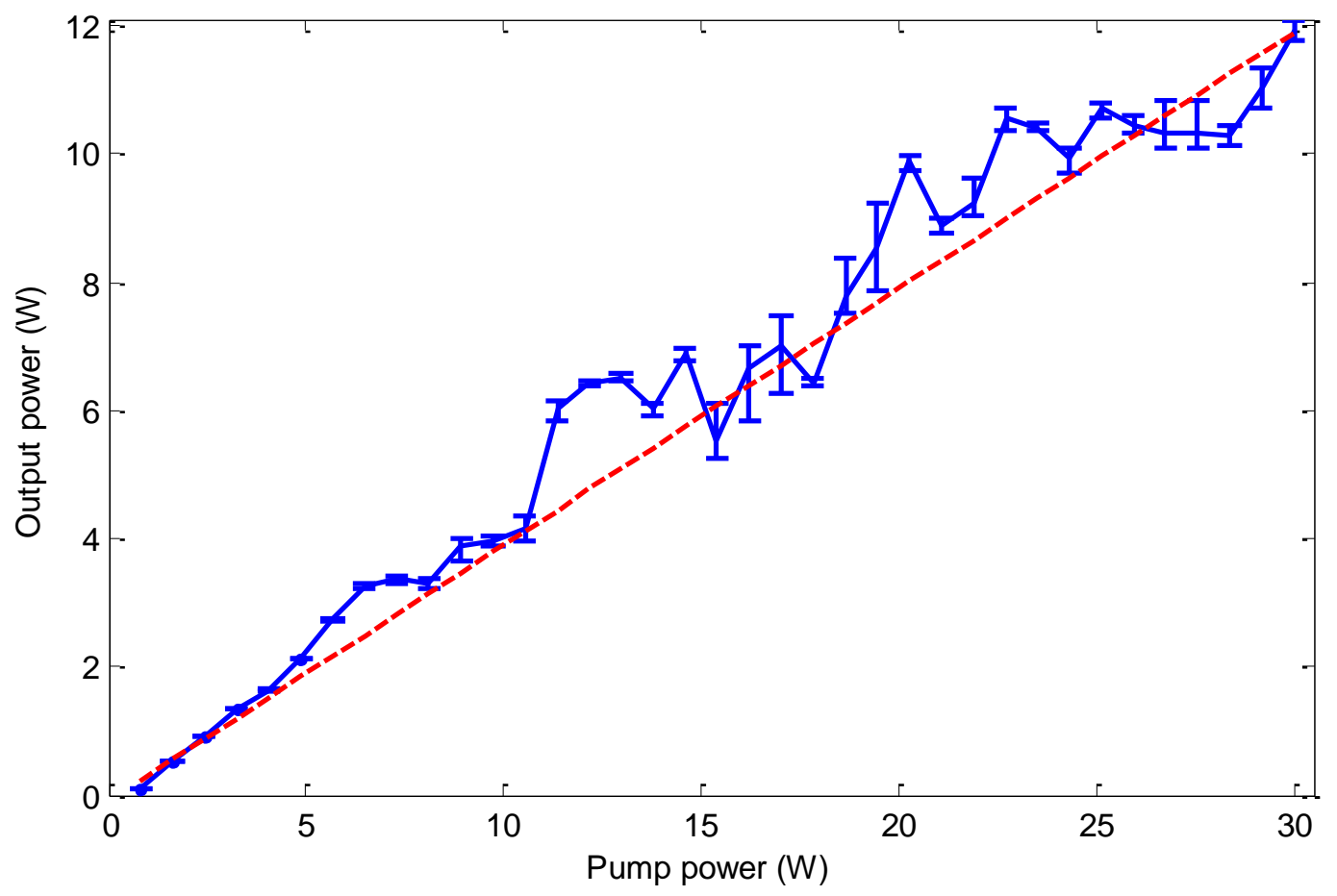

Figure 30: Output power vs incident pump power for single-leg Z-cavity. The average slope efficiency is $\sim 40 \%$. The error bars show the minimum and maximum of 1000 measurements of output power per second at each data point. The output power oscillation and random non-linearity of the curve are attributed to thermal lensing instability and the random pump beam distortions caused by atmospheric water vapor absorption of the pump radiation.

To address these concerns, a double collimated-leg Z-cavity was assembled as illustrated with the solid elements in Figure 29. The same $5 \mathrm{~cm}$ ROC mirror (M1) used as an end mirror in the above cavity was moved one focal length from the gain element and used as the input coupler while also creating the second collimated leg. The $\sim 16 \mathrm{~cm}$ long 'functional element' (FE) leg was terminated with a planar HR mirror for free- 
running mode or a $300 \mathrm{gr} / \mathrm{mm}, 2.5 \mu \mathrm{m}$ blazed grating in the Littrow configuration for tuning and line narrowing. It was found experimentally that the optimal pump lens (LP) focal length was $7.5 \mathrm{~cm}$. Modeling cavity mode overlap with the pump can be accomplished using a standard ABCD matrix method, but the results were merely a good starting point experimentally due to the variable and strong nature of the thermal lensing.

When robust AR coatings are available, some of the astigmatism problems can be eliminated by utilizing a normal-incidence $L$-cavity pumping scheme. This simplifies the cavity considerably as seen in Figure 31A. Cavity stability can be achieved using either a concave HR end mirror ( $L_{a}$-cavity) or a planar HR end mirror with an intracavity AR coated positive lens ( $L_{b}$-cavity). The latter configuration is more complicated to construct in confined quarters and may have some loss depending on the quality of the AR coating but M2 could be replaced with a grating in the Littrow configuration to achieve cavity tuning.

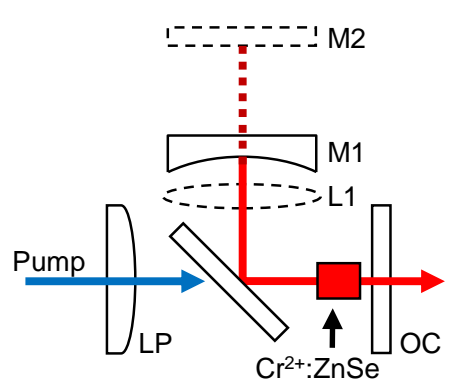

A)

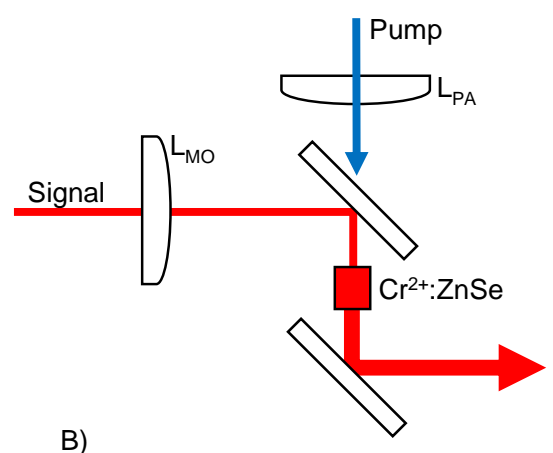

B)

Figure 31: A) Normal-incidence $L$-cavity scheme which can use either an HR curved mirror (M1) or a combination of planar HR (M2) and positive lens (L1). B) Power amplifier design where $L_{M O}$ and $L_{P A}$ are chosen for optimal overlap of pump and signal beams.

The power amplifier section used a co-linearly pumped, polycrystalline, 8.9x7.0x3.0 $\mathrm{mm}(\mathrm{LxWxH}) \mathrm{Cr}^{2+}: \mathrm{ZnSe}$ crystal similar to the MO crystal, but AR coated for $1.9-3 \mu \mathrm{m}$. As seen in Figure 31B, the $150 \mathrm{~W}$ maximum, $1.9 \mu \mathrm{m}$, Tm-fiber laser pump beam is co- 
aligned with the MO output. Both beams are focused for optimal mode overlap of around $80 \mu \mathrm{m}$ radius with $15 \mathrm{~cm}$ focal length lenses ( $\mathrm{L}_{\mathrm{MO}}$ and $\mathrm{L}_{\mathrm{PA}}$ ).

\subsection{Experimental Results}

The $Z$ - and $L$-cavity oscillators were optimized for highest output at the upper limit of their stability region (Z-cavity) or just below the damage threshold for the AR coatings ( $L$-cavities). All experiments were done with the laser samples cooled with circulating water maintained at $10^{\circ} \mathrm{C}$. Experimentally, it was determined that the $L_{b}$-cavity was easily out-performed by the $L_{a}$-cavity, possibly due to additional losses from the intracavity lens. Once an MO was optimized in this way, the PA pump power was then varied to test the system and compare to the theoretical model. The data shown in Figure 32 represents the best performance of each of the MOPA configurations. From this, it can be seen that the $L_{a}$-cavity is better suited for power scaling than the $Z$-cavity. This is likely due to the limited ability of the fixed-angle curved mirrors in the Z-cavity to compensate for the increasing astigmatism from the Brewster-angle thermal lens in the gain element. However, it should be noted that the output from the $Z$-cavity is tunable and, due to the poor performance of the $L_{b}$-cavity, this is not an option in the linear cavity configuration. The experimental data follows the model-predicted performance at first but then under-performs the predictions. This could be due to thermal lensing in the amplifier creating non-optimal overlap between the pump and signal. Both signal and pump should, in general, experience the same thermal lensing but dispersion could result in small differences in lensing power. Experimentally, it was noticed that any small deviations in overlap resulted in significant output reduction, so even minute lensing variations could result in the observed differences between model and experiment. 


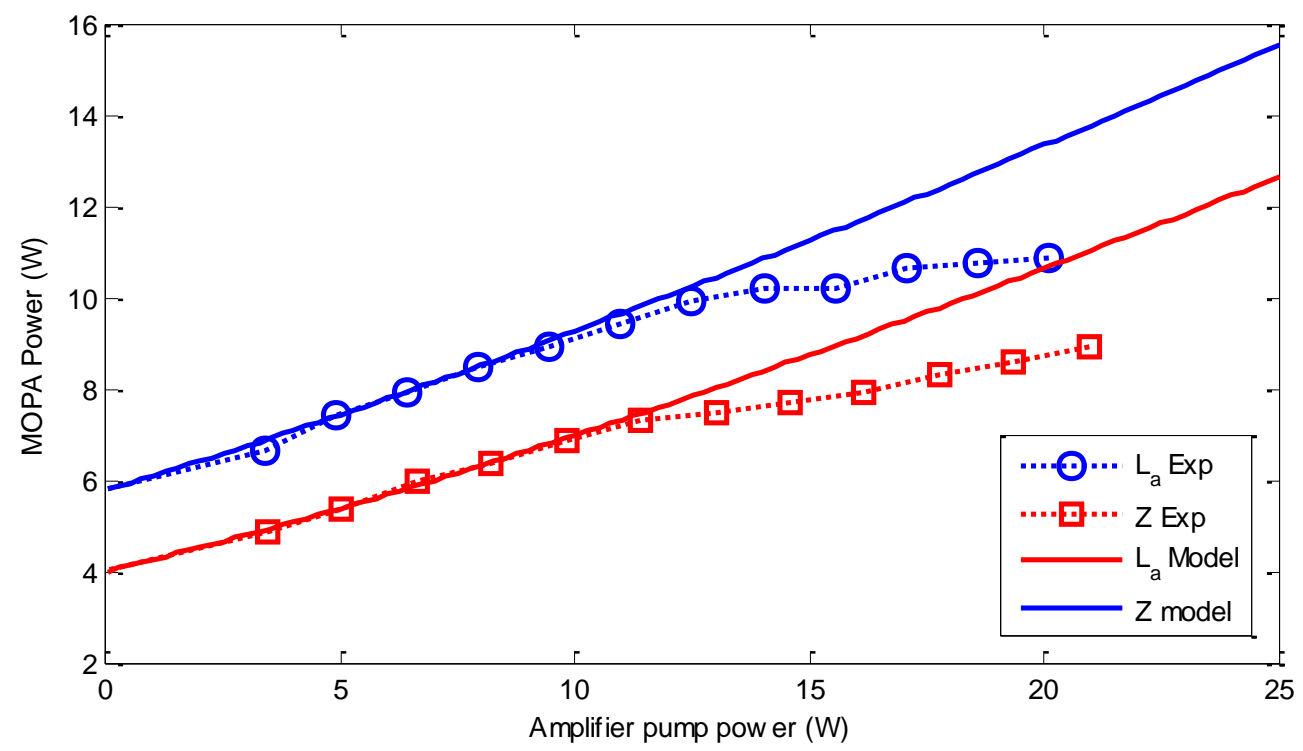

Figure 32: Power scaling ability of MOPA configurations. Experimental data (discrete points) is compared with model predictions for $L_{a}$ - and $Z$-cavities, both with $100 \mu \mathrm{m}$ spot sizes.

The output spectrum of the free-running (no grating present) $L_{b}$-cavity MO was measured as a function of output power. Spectra were measured by a $2 \mathrm{MHz}$ Cal Sensors 256 element $\mathrm{PbSe}$ linear detector array coupled to the exit slit of a $750 \mathrm{~mm}$ Acton Research Corporation monochromator. This system was calibrated and optimized using a Yokogawa 735305 optical spectrum analyzer (OSA) and a $150 \mathrm{~W}$ IPG Tm fiber laser. The OSA showed a $0.2 \mathrm{~nm}$ linewidth for the $150 \mathrm{~W}$ IPG laser and after proper positioning of the array at the focal plane and adjustment of the monochromator slit widths, similar resolution was achieved with the spectrometer system. Figure 33 shows that the width of the collection of output peaks of the laser is not a function of pump power; however, the weighted average wavelength increases with pump power, going from 2415 to $2442 \mathrm{~nm}$. There are a number of thermal effects which could be postulated to shift the average output wavelength in either direction, but it is difficult to predict which will dominate. A shift to the longer wavelengths could be due to higher temperatures in the crystal affecting non-radiative transitions for higher energy levels. 


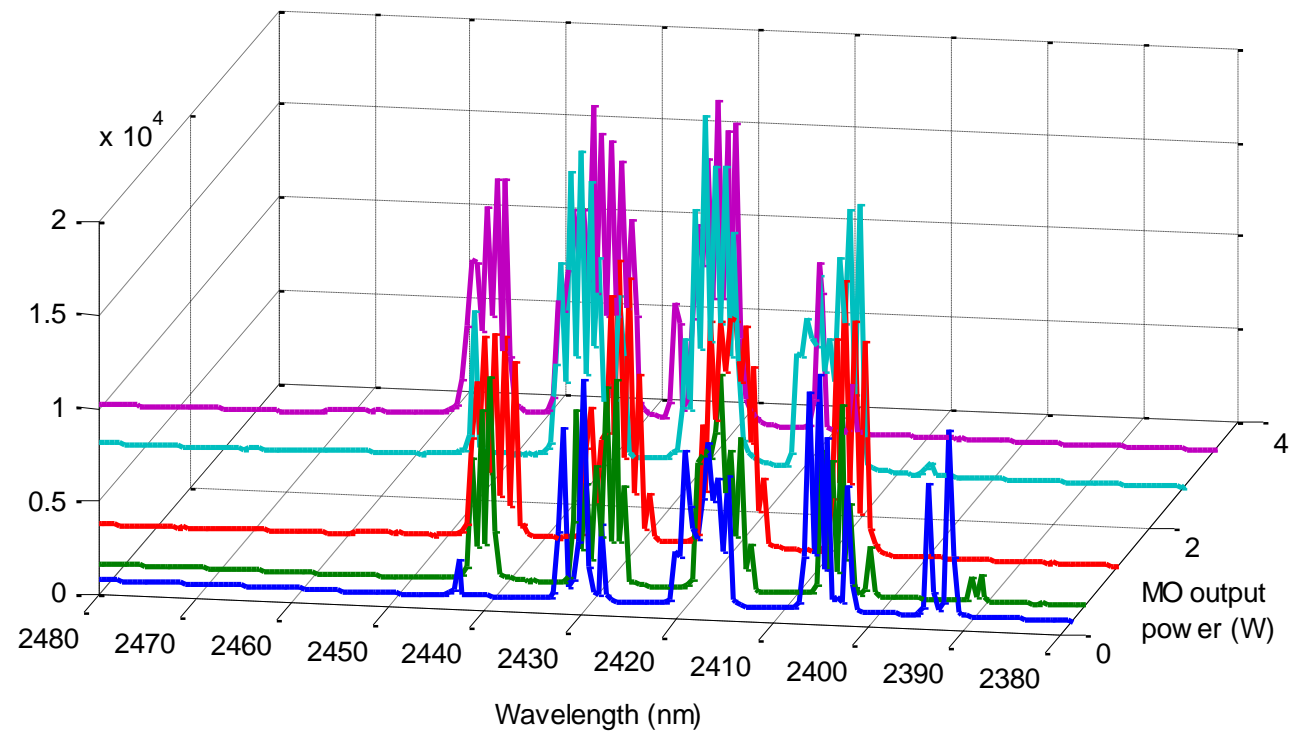

Figure 33: Output spectrum of free-running $L_{b}$-cavity as a function of output power showing a shift to higher wavelengths at increased power.

Representative tunable output characteristics of the amplified, grating equipped Zcavity are shown in Figure 34. A maximum power of $8.9 \mathrm{~W}$ was achieved at $2450 \mathrm{~nm}$ with tunable lasing over the $2275-2700 \mathrm{~nm}$ range, which was limited by grating efficiency and wavelength-dependent coating reflectivity. Figure 34 shows the dependence of the measured output power on wavelength superimposed with the linewidths measured at the same points, which were on the order of $2 \mathrm{~nm}$. 


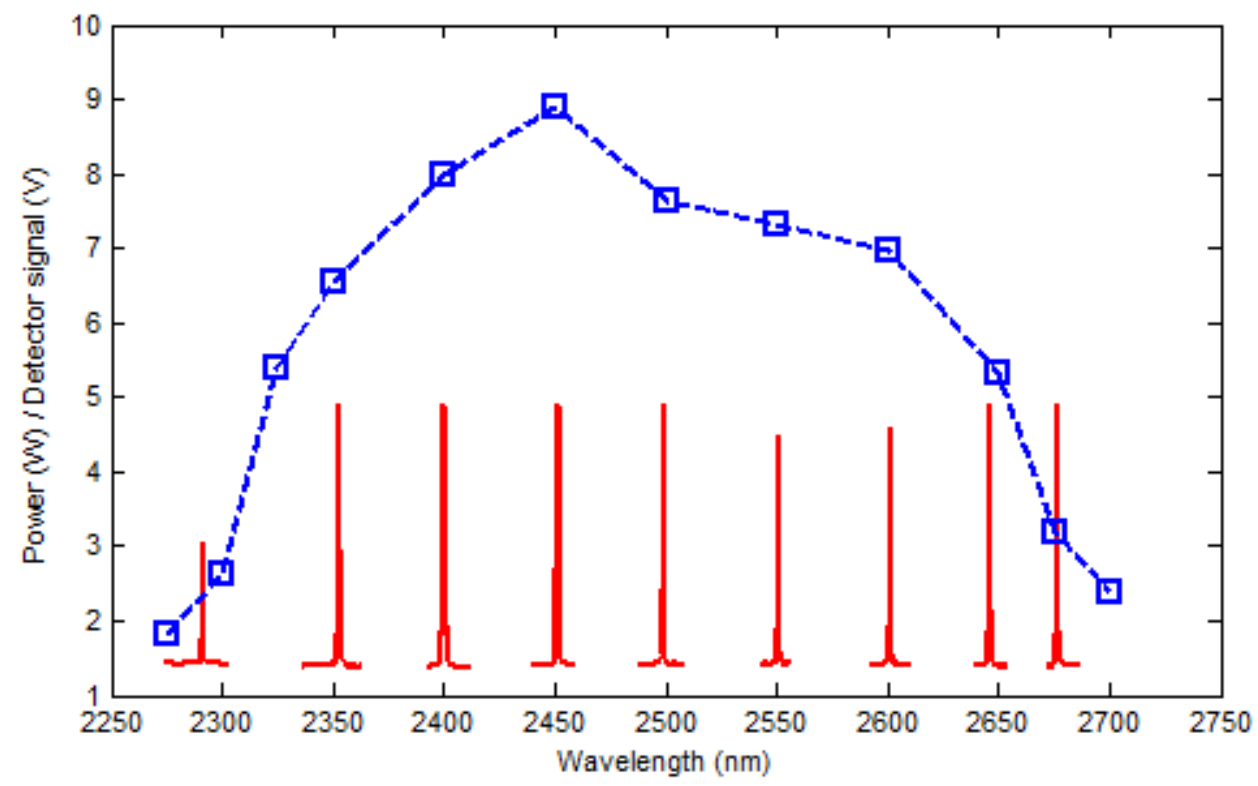

Figure 34: Tunable output power of $Z$-cavity with representative linewidth of output.

One of the goals of the MOPA configuration is an improvement in the beam quality for a given output power. The need for this can be understood by looking at the nearfield beam profile of the MO output. Figure 35 shows a single-frame excerpt from a video showing the near-field beam profile of the $L_{b}$-cavity as a function of decreasing pump power. The cavity will run stable in one mode $\left(\mathrm{TEM}_{00}\right)$ at low powers until the power changes enough to allow or cause a run in combinations of modes with the best stability. This type of transition period in the cavity is marked by unstable and fluctuating output powers and beam quality. On the other hand, with a MOPA system, the MO could be placed in a stable region while the system power output is varied by the PA, allowing for more stable overall output power and quality. 

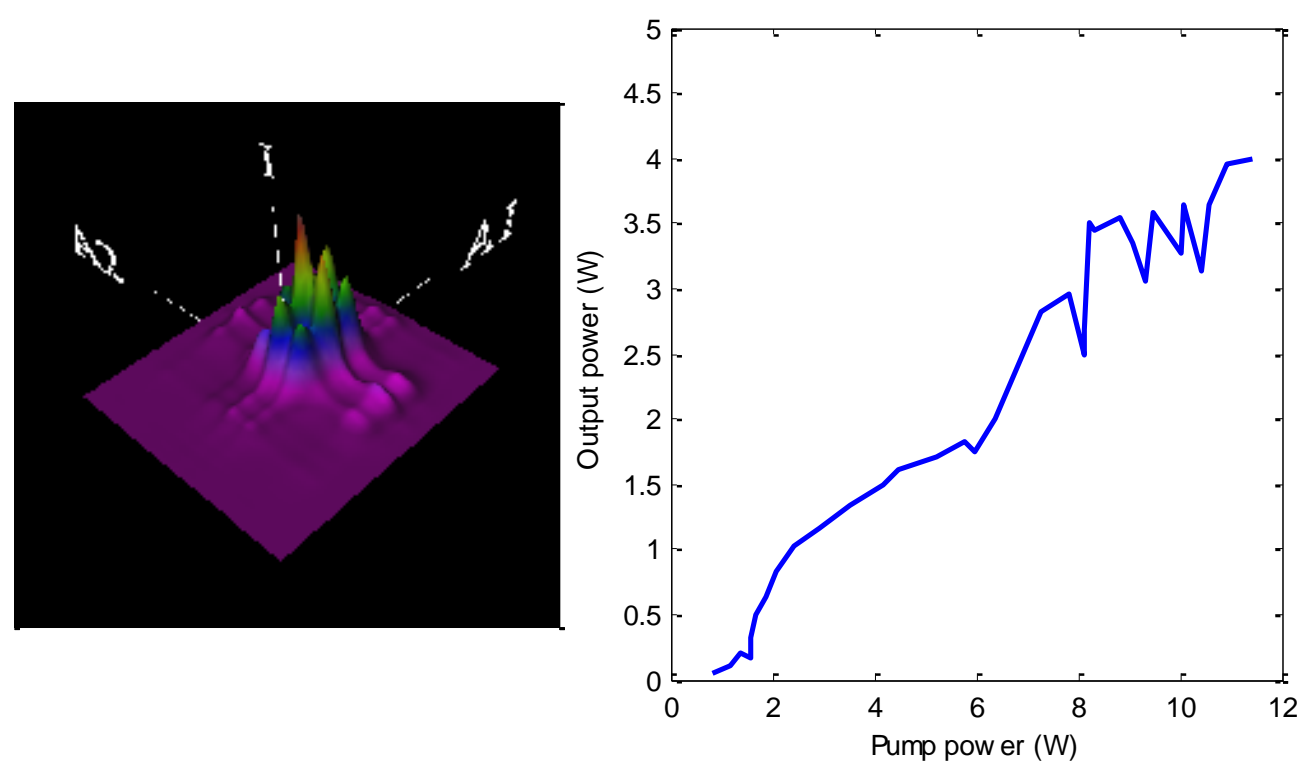

Figure 35: Single-frame excerpt (left) from video showing near-field beam profile changes of $L_{b}$ cavity as a function of decreasing pump power. Movie starts with maximum pump power and ends at zero pump power. Transitions between different modes cause instability in power output (right).

The ability of the MOPA system to achieve higher output powers while maintaining beam quality was tested by measuring the $\mathrm{M}^{2}$ of the $L_{a}$-cavity. At $1 \mathrm{~W}$, the cavity had an $\mathrm{M}^{2}$ of 1.2 and at $3 \mathrm{~W}$ it had an $\mathrm{M}^{2}$ of 1.8 . With the $\mathrm{MO}$ running at $1 \mathrm{~W}$ and amplified to $3 \mathrm{~W}$ with the PA, the system had an $\mathrm{M}^{2}$ of 1.3 . This supports the claim that a MOPA system can be used to power scale a system where other issues may limit oscillator operation.

\subsection{Conclusions}

In conclusion, we have demonstrated a $\mathrm{CW} \mathrm{Cr}^{2+}: \mathrm{ZnSe}$ MOPA laser with output power over $10 \mathrm{~W}$ and amplifier gain of $2 \mathrm{X}$. We developed an amplifier model for co-linearly pumped $\mathrm{Cr}^{2+}: \mathrm{ZnSe}$, which compares well with our experimental data. Use of a MOPA configuration allowed us to demonstrate high-power and good beam quality in a $\mathrm{Cr}^{2+}: \mathrm{ZnSe}$ laser. In addition, we demonstrated single-knob tunability at multi-watt powers over a $400 \mathrm{~nm}$ tuning range. 
As is common with chromium-doped II-VI lasers, thermal effects limited the output power and beam quality of this setup. Improvements in cooling are needed to take full advantage of the available pump power and increase system stability. This MOPA system will be evaluated with addition MO configurations to study the optimal operating conditions and increase output laser power. Thermal management techniques will continue to be improved in an effort to reduce thermal instability in the oscillator. 


\section{Chapter 6}

\section{Conclusions}

\subsection{Summary}

In this research a number of approaches were used to understand and mitigate thermal issues limiting the level to which tunable $\mathrm{Cr}^{2+}: \mathrm{ZnSe}$ lasers could be power scaled. It was found that careful consideration of heat removal, lensing compensation and system architecture can help remove many of the hurdles to high-power operation. A finiteelement analysis model was used to help understand the time-evolution of the thermal lensing problem. Crucial initial work in the development of fiber-based $\mathrm{Cr}^{2+}: \mathrm{ZnSe}$ lasers was completed and, while not successful in producing a $\mathrm{Cr}^{2+}: \mathrm{ZnSe}$ fiber, helped understand the material problems associated with this geometry. $\mathrm{A} \mathrm{Cr}^{2+}: \mathrm{ZnSe}$ laser was constructed which demonstrated over $12 \mathrm{~W}$ of pure $\mathrm{CW}$ output power, doubling the previous record. A model for amplification of co-linearly pumped master oscillator power amplifier systems was developed and used to design the first demonstrated $\mathrm{Cr}^{2+}: \mathrm{ZnSe}$ MOPA systems with greater than $10 \mathrm{~W}$ pure $\mathrm{CW}$ power and $9 \mathrm{~W}$ tunable over $400 \mathrm{~nm}$.

\subsection{Future Work}

This work showed that there are still a number of things that can be done to power scale these lasers. Using thermal-gradient reducing geometries like zig-zag slab lasers is 
planned to further increase output powers. The fiber laser work has lead to a better understanding of the difficulties involved in producing a $\mathrm{Cr}^{2+}: \mathrm{ZnSe}$ fiber laser. This has lead into new efforts to produce these types of waveguides which hold promise. The MOPA system design will also be used to produce high-power modelocked pulses for use in pumping nonlinear devices. 


\section{References}

1. K. L. Schepler, and P. A. Berry, "A promising mid-infrared transition metal laser ion," in SPIE Newsroom(2007).

2. K. L. Schepler, R. D. Peterson, P. A. Berry, and J. B. McKay, "Thermal Effects in $\mathrm{Cr}^{2+}: \mathrm{ZnSe}$ Thin Disk Lasers," IEEE Journal of Selected Topics in Quantum Electronics 11, 713-720 (2005).

3. P. A. Berry, and K. L. Schepler, "Modeling of time-dependent thermal effects in Cr[sup $2+$ ]-doped zinc selenide thin disks," in Solid State Lasers XV: Technology and Devices(SPIE, San Jose, CA, USA, 2006), pp. 61000X-61010.

4. I. S. Moskalev, V. V. Fedorov, S. B. Mirov, P. A. Berry, and K. L. Schepler, "12Watt CW Polycrystalline $\mathrm{Cr}^{2+}: \mathrm{ZnSe}$ Laser Pumped by Tm-fiber Laser," in Advanced Solid State Photonics(Optical Society of America, Denver, CO, 2008), p. WB30.

5. P. A. Berry, and K. L. Schepler, $" \mathrm{Cr}^{2+}: \mathrm{ZnSe}$ master oscillator / power amplifier for improved power scaling," in Solid State Lasers XIX: Technology and Devices(SPIE, San Francisco, California, USA, 2010), pp. 75781L-75711.

6. P. A. Berry, and K. L. Schepler, "High-power, widely-tunable $\mathrm{Cr}^{2+}: \mathrm{ZnSe}$ master oscillator power amplifier systems," Optics Express (Submitted 2010).

7. $\quad$ P. A. Berry, and K. L. Schepler, "High-Power, Widely Tunable $\mathrm{Cr}^{2+}: \mathrm{ZnSe}$ Laser," in Conference on Lasers and Electro-Optics(Optical Society of America, San Jose, CA, 2010).

8. Y. Jong-In, S. Paula, M. P. George, and V. Vasan, "Mid-IR laser ablation of articular and fibro-cartilage:A wavelength dependence study of thermal injury and crater morphology," Lasers in Surgery and Medicine 38, 218-228 (2006).

9. B. Jean, and T. Bende, "Mid-IR Laser Applications in Medicine," in Solid-State Mid-Infrared Laser Sources(2003), pp. 530-565.

10. A. L. Schawlow, and C. H. Townes, "Infrared and Optical Masers," Physical Review 112, 1940 (1958).

11. T. H. Maiman, "Stimulated Optical Radiation in Ruby," Nature 187, 493-494 (1960). 
12. J. E. Geusic, H. M. Marcos, and L. G. Van Uitert, "Laser Oscillations In NdDoped Yttrium Aluminum, Yttrium Gallium And Gadolinium Garnets," Applied Physics Letters 4, 182-184 (1964).

13. K. S. Bagdasarov, and A. A. Kaminskii, "YAlO ${ }_{3}$ with $\mathrm{TR}^{3+}$ Ion Impurity as an Active Laser Medium," JETP Letters 9, 303 (1969).

14. E. V. Zharikov, and et al., "Stimulated emission from $\mathrm{Er}^{3+}$ ions in yttrium aluminum garnet crystals at lambda $=2.94$ microns," Soviet Journal of Quantum Electronics 4, 1039 (1975).

15. N. Barnes, and D. Gettemy, "Pulsed Ho:YAG oscillator and amplifier," IEEE J. Quantum Electron. 17, 1303 (2003).

16. E. Snitzer, and C. G. Young, "Glass lasers," Applied Optics 5, 1487-1499 (1966).

17. B. Henderson, and R. H. Bartram, Crystal field engineering of solid state laser materials (Cambridge Univ Pr, 2000).

18. B. Henderson, and G. F. Imbusch, Optical Spectroscopy of Inorganic Solids (Clarendon Press, Oxford, 1989).

19. K. L. Schepler, "Mid-IR transition metal lasers," in Solid State Lasers XVI: Technology and Devices(SPIE, San Jose, CA, USA, 2007), pp. 64510K-64511.

20. L. F. Johnson, R. E. Dietz, and H. J. Guggenheim, "Optical Maser Oscillation from $\mathrm{Ni}^{2+}$ in $\mathrm{MgF} 2$ Involving Simultaneous Emission of Phonons," Physical Review Letters 11, 318 (1963).

21. L. F. Johnson, R. E. Dietz, and H. J. Guggenheim, "Spontaneous And Stimulated Emission From $\mathrm{Co}^{2+}$ Ions In $\mathrm{MgF}_{2}$ And $\mathrm{ZnF}_{2}$," Applied Physics Letters 5, 21-22 (1964).

22. P. F. Moulton, A. Mooradian, and T. B. Reed, "Efficient cw optically pumped Ni:MgF 2 laser," Opt. Lett. 3, 164-166 (1978).

23. P. F. Moulton, "An investigation of the Co: $\mathrm{MgF}_{2}$ laser system," IEEE J. Quantum Electron. 21, 1582-1595 (1985).

24. M. V. Iverson, and W. A. Sibley, "Temperature dependence of $\mathrm{Ni2}+$ luminescence in $\mathrm{KZnF} 3, \mathrm{MgF} 2$ and MgO," Journal of Luminescence 20, 311-324 (1979).

25. R. H. Page, and E. al., "Cr${ }^{2+}$-doped II-VI crystals: new widely tunable, roomtemperature mid-IR lasers," in Lasers and Electro-Optics Society Annual Meeting(IEEE, San Francisco, CA, 1995).

26. R. H. Page, L. D. DeLoach, K. I. Schaffers, F. D. Patel, R. J. Beach, S. A. Payne, W. F. Krupke, and A. Burger, "Recent developments in $\mathrm{Cr}^{2+}$-doped II-VI compound lasers," in Advanced Solid State Lasers(Optical Society of America, 1996), p. TL16.

27. L. D. DeLoach, R. H. Page, G. D. Wilke, S. A. Payne, and W. F. Krupke, "Transition metal-doped zinc chalcogenides: spectroscopy and laser 
demonstration of a new class of gain media," IEEE J. Quantum Electron. 32, 885895 (1996).

28. R. H. Page, K. I. Schaffers, L. D. DeLoach, G. D. Wilke, F. D. Patel, J. B. Tassano, Jr., S. A. Payne, W. F. Krupke, K. T. Chen, and A. Burger, "Cr ${ }^{2+}$-doped zinc chalcogenides as efficient, widely tunable mid-infrared lasers," IEEE J. Quantum Electron. 33, 609-619 (1997).

29. I. T. Sorokina, E. Sorokin, A. Di Lieto, M. Tonelli, R. H. Page, and K. I. Schaffers, "Efficient broadly tunable continuous-wave $\mathrm{Cr}^{2+}$ :ZnSe laser," J. Opt. Soc. Am. B 18, 926-930 (2001).

30. I. T. Sorokina, E. Sorokin, S. Mirov, V. Fedorov, V. Badikov, V. Panyutin, and K. I. Schaffers, "Broadly tunable compact continuous-wave $\mathrm{Cr}^{2+}: \mathrm{ZnS}$ laser," Opt. Lett. 27, 1040-1042 (2002).

31. U. Hömmerich, X. Wu, V. R. Davis, S. B. Trivedi, K. Grasza, R. J. Chen, and S. Kutcher, "Demonstration of room-temperature laser action at $2.5 \mu \mathrm{m}$ from $\mathrm{Cr}^{2+}: \mathrm{Cd}_{0.85} \mathrm{Mn}_{0.15}$ Te," Opt. Lett. 22, 1180-1182 (1997).

32. U. Hömmerich, J. T. Seo, A. Bluiett, M. Turner, D. Temple, S. B. Trivedi, H. Zong, S. W. Kutcher, C. C. Wang, R. J. Chen, and B. Schumm, "Mid-infrared laser development based on transition metal doped cadmium manganese telluride," Journal of Luminescence 87-89, 1143-1145 (2000).

33. J. McKay, K. L. Schepler, and G. C. Catella, "Efficient grating-tuned mid-infrared $\mathrm{Cr}^{2+}$ :CdSe laser," Opt. Lett. 24, 1575-1577 (1999).

34. K. L. Schepler, S. Kück, and L. Shiozawa, "Cr ${ }^{2+}$ emission spectroscopy in CdSe," Journal of Luminescence 72-74, 116-117 (1997).

35. U. Demirbas, and A. Sennaroglu, "Intracavity-pumped $\mathrm{Cr}^{2+}: \mathrm{ZnSe}$ laser with ultrabroad tuning range between 1880 and 3100 nm," Opt. Lett. 31, 2293-2295 (2006).

36. E. Sorokin, I. T. Sorokina, M. S. Mirov, V. V. Fedorov, I. S. Moskalev, and S. B. Mirov, "Ultrabroad Continuous-Wave Tuning of Ceramic Cr:ZnSe and Cr:ZnS Lasers," in Advanced Solid-State Photonics(Optical Society of America, 2010), p. AMC2.

37. R. D. Peterson, and K. L. Schepler, "1.9 micron-Fiber-Pumped Cr:ZnSe Laser," in Advanced Solid-State Photonics 2005 Technical Digest on CD-ROM(The Optical Society of America, Washington, DC, 2005), p. MB13.

38. R. H. Page, J. A. Skidmore, K. I. Schaffers, R. J. Beach, S. A. Payne, and W. F. Krupke, "Demonstrations of diode - pumped and grating - tuned $\mathrm{ZnSe}: \mathrm{Cr}^{2+}$ lasers," in Advanced Solid State Lasers(Optical Society of America, 1997), p. LS6.

39. N. Kuleshov, M. Mond, E. Heumann, H. Kretschmann, S. Kueck, G. Huber, V. I. Levchenko, and V. N. Yakimovich, "Continuous wave diode pumped $\mathrm{Cr}^{2+}: \mathrm{ZnSe}$ and high power laser operation," in Advanced Solid-State Lasers(Optical Society of America, 2001), p. MC3. 
40. G. J. Wagner, T. J. Carrig, R. H. Page, K. I. Schaffers, J.-O. Ndap, X. Ma, and A. Burger, "Continuous-wave broadly tunable $\mathrm{Cr}^{2+}$ :ZnSe laser," Opt. Lett. 24, 19-21 (1999).

41. T. J. Carrig, G. J. Wagner, W. J. Alford, and A. Zakel, "Chromium-doped chalcogenide lasers," in Solid State Lasers and Amplifiers, A. Sennaroglu, J. G. Fujimoto, and C. R. Pollock, eds. (SPIE, Bellingham, WA, 2004), pp. 74-82.

42. G. J. Wagner, B. G. Tiemann, W. J. Alford, and T. J. Carrig, "Single-Frequency Cr:ZnSe Laser," in Advanced Solid-State Photonics(Optical Society of America, 2004), p. WB12.

43. I. S. Moskalev, V. V. Fedorov, and S. B. Mirov, "10-Watt, pure continuous-wave, polycrystalline $\mathrm{Cr}^{2+}: \mathrm{ZnS}$ laser," Opt. Express 17, 2048-2056 (2009).

44. R. J. Harris, G. T. Johnston, G. A. Kepple, P. C. Krok, and H. Mukai, "Infrared thermooptic coefficient measurement of polycrystalline $\mathrm{ZnSe}, \mathrm{ZnS}, \mathrm{CdTe}, \mathrm{CaF}_{2}$, and $\mathrm{BaF}_{2}$, single crystal KCI, and TI-20 glass," Appl. Opt. 16, 436-438 (1977).

45. D. M. Simanovskii, H. A. Schwettman, H. Lee, and A. J. Welch, "Midinfrared Optical Breakdown in Transparent Dielectrics," Physical Review Letters 91, 107601 (2003).

46. I. T. Sorokina, "Cr ${ }^{2+}$-doped II-VI materials for lasers and nonlinear optics," Optical Materials 26, 395-412 (2004).

47. P. A. Berry, "Thermal Lens Mitigation and Modeling of Chromium-doped Zinc Selenide Laser Sources," in School of Engineering(University of Dayton, Dayton, $\mathrm{OH}, 2005)$.

48. R. C. Weast, ed. Handbook of Chemistry and Physics (CRC Press, Inc, Boca Raton, FL, 1988).

49. Y.-C. Huang, Y.-K. Lu, J.-C. Chen, Y.-C. Hsu, Y.-M. Huang, H.-M. Yang, M.-T. Sheen, S.-L. Huang, T.-Y. Chang, and W.-H. Cheng, "Fabrication of Cr-Doped Fibers by Drawing Tower," in Optical Fiber Communication Conference and Exposition and The National Fiber Optic Engineers Conference(Optical Society of America, 2006), p. OWI21.

50. Y. C. Huanga, Y. K. Lua, J. C. Chena, Y. C. Hsub, Y. M. Huanga, S. L. Huanga, and W. H. Chenga, "Broadband emission from Cr-doped fibers fabricated by drawing tower," system 36, 183-184 (2006).

51. Y. Vaksman, Y. Nitsuk, V. Yatsun, A. Nasibov, and P. Shapkin, "Preparation and optical properties of ZnSe:Ni crystals," Semiconductors 44, 141-144.

52. P. J. A. Sazio, A. Amezcua-Correa, C. E. Finlayson, J. R. Hayes, T. J. Scheidemantel, N. F. Baril, B. R. Jackson, D.-J. Won, F. Zhang, E. R. Margine, V. Gopalan, V. H. Crespi, and J. V. Badding, "Microstructured Optical Fibers as High-Pressure Microfluidic Reactors," Science 311, 1583-1586 (2006).

53. N. D. Psaila, R. R. Thomson, H. T. Bookey, A. K. Kar, N. Chiodo, R. Osellame, G. Cerullo, G. Brown, A. Jha, and S. Shen, "Femtosecond laser inscription of optical waveguides in Bismuth ion doped glass," Phys. Lett 88, 191912 (2006). 
54. J. B. McKay, W. B. Roh, and K. L. Schepler, "4.2 $\mathrm{W} \mathrm{Cr}^{2+}$ :ZnSe face cooled disk laser," in OSA Trends in Optics and Photonics vol. 73, Conference on Lasers and Electro-Optics(Optical Society of America, Washington DC, 2002), pp. 119-120.

55. J. Dieleman, R. S. Title, and W. V. Smith, "Paramagnetic resonance studies of $\mathrm{Cr}+$ in cubic and hexagonal ZnS," Physics Letters 1, 334-335 (1962).

56. G. Grebe, and et al., "Cr 2+ excitation levels in $\mathrm{ZnSe}$ and $\mathrm{ZnS}$," Journal of Physics C: Solid State Physics 9, 4511 (1976).

57. J. B. McKay, "Power scaling feasibility of chromium-doped ii-vi laser sources and the demonstration of a chromium-doped zinc selenide face-cooled disk laser.," in Graduate School of Engineering and Management(Air Force Institute of Technology, Air University, Air Education and Training Command, 2003).

58. A. Sennaroglu, U. Demirbas, A. Kurt, and M. Somer, "Concentration dependence of fluorescence and lasing efficiency in $\mathrm{Cr}^{2+}: \mathrm{ZnSe}$ lasers," Optical Materials 29, 703-708 (2007).

59. H. Kogelnik, E. Ippen, A. Dienes, and C. Shank, "Astigmatically compensated cavities for CW dye lasers," IEEE J. Quantum Electron. 8, 373-379 (1972). 


\section{Appendix 1}

\section{Listing of MATLAB Codes}

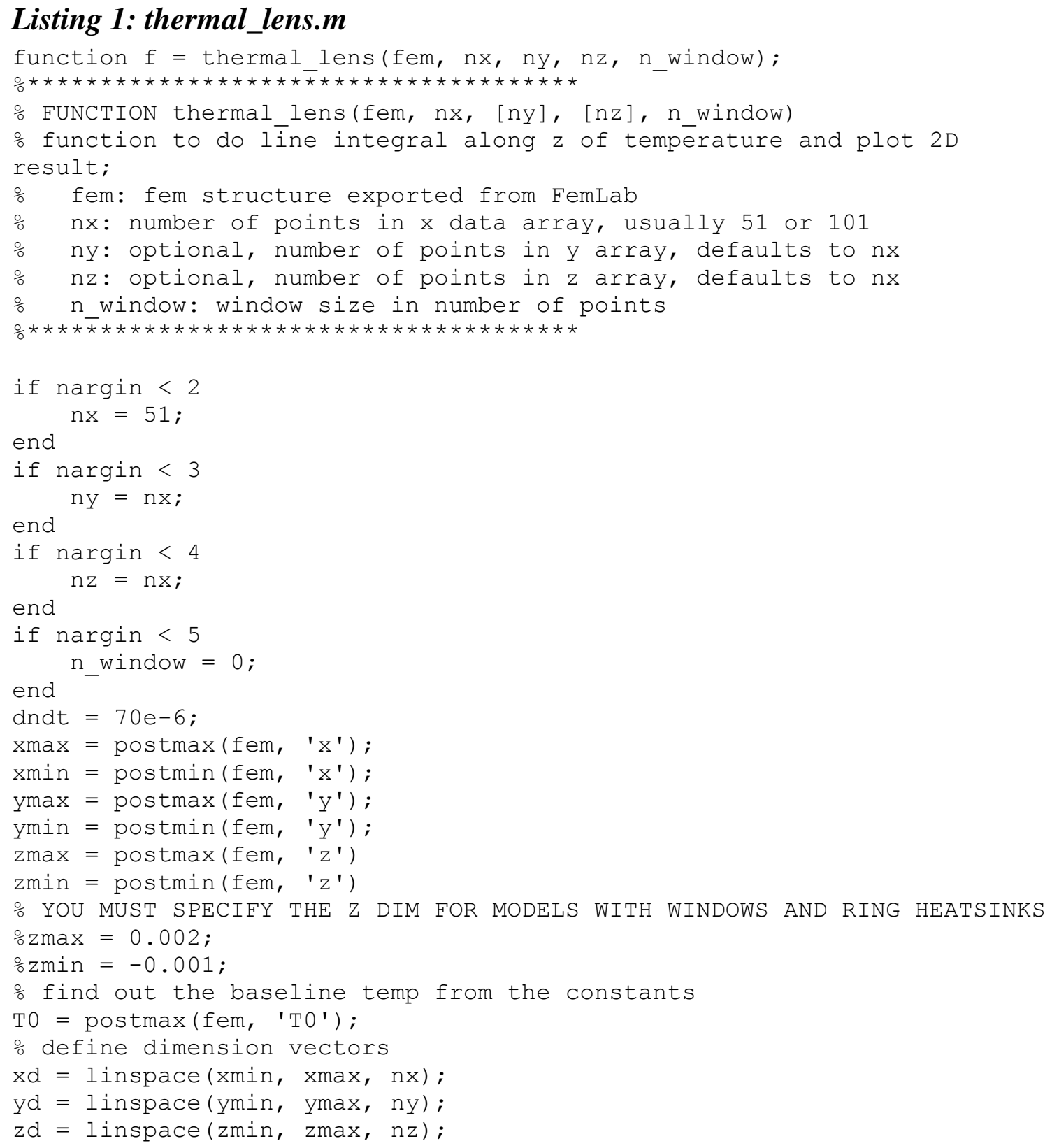




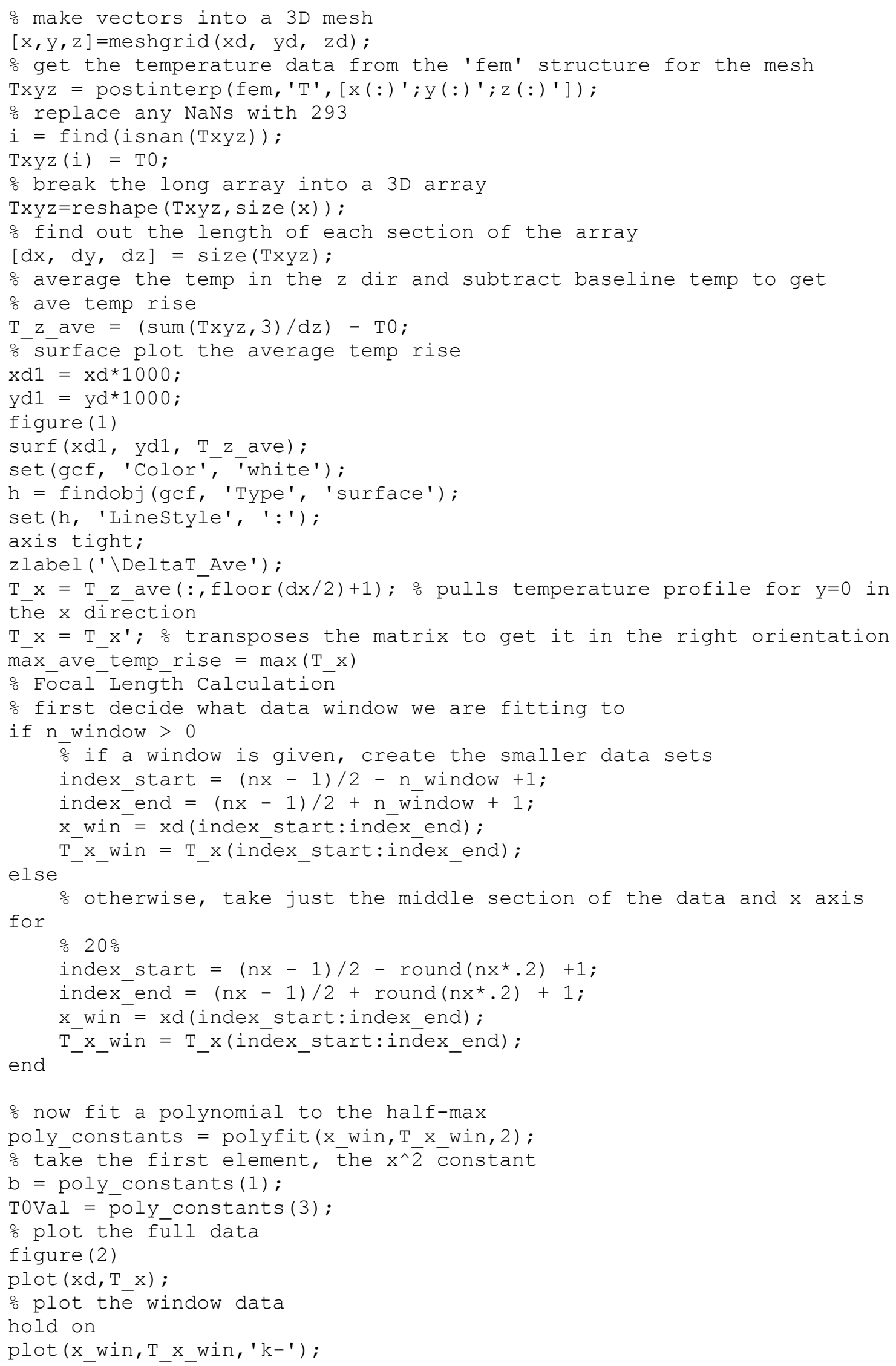




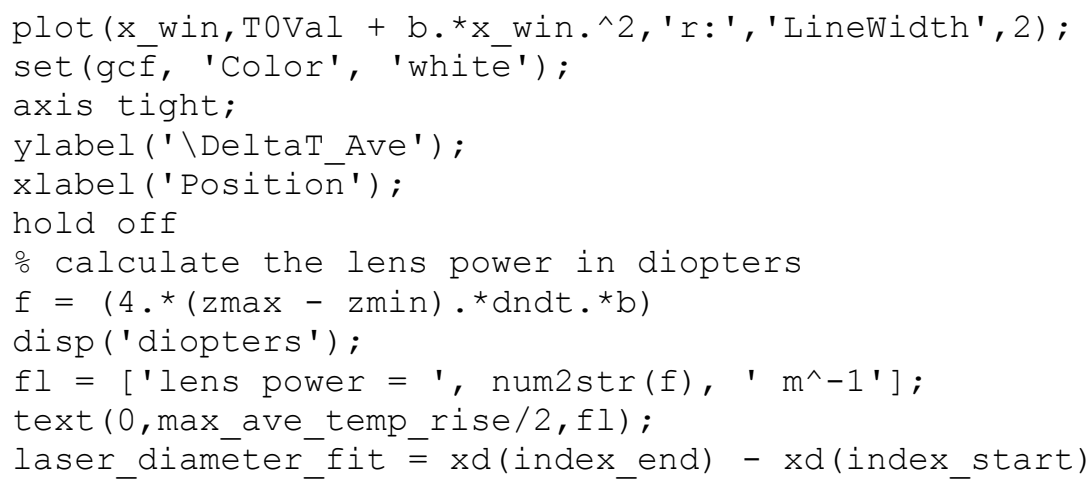




\section{Listing 2: HSIC.m}

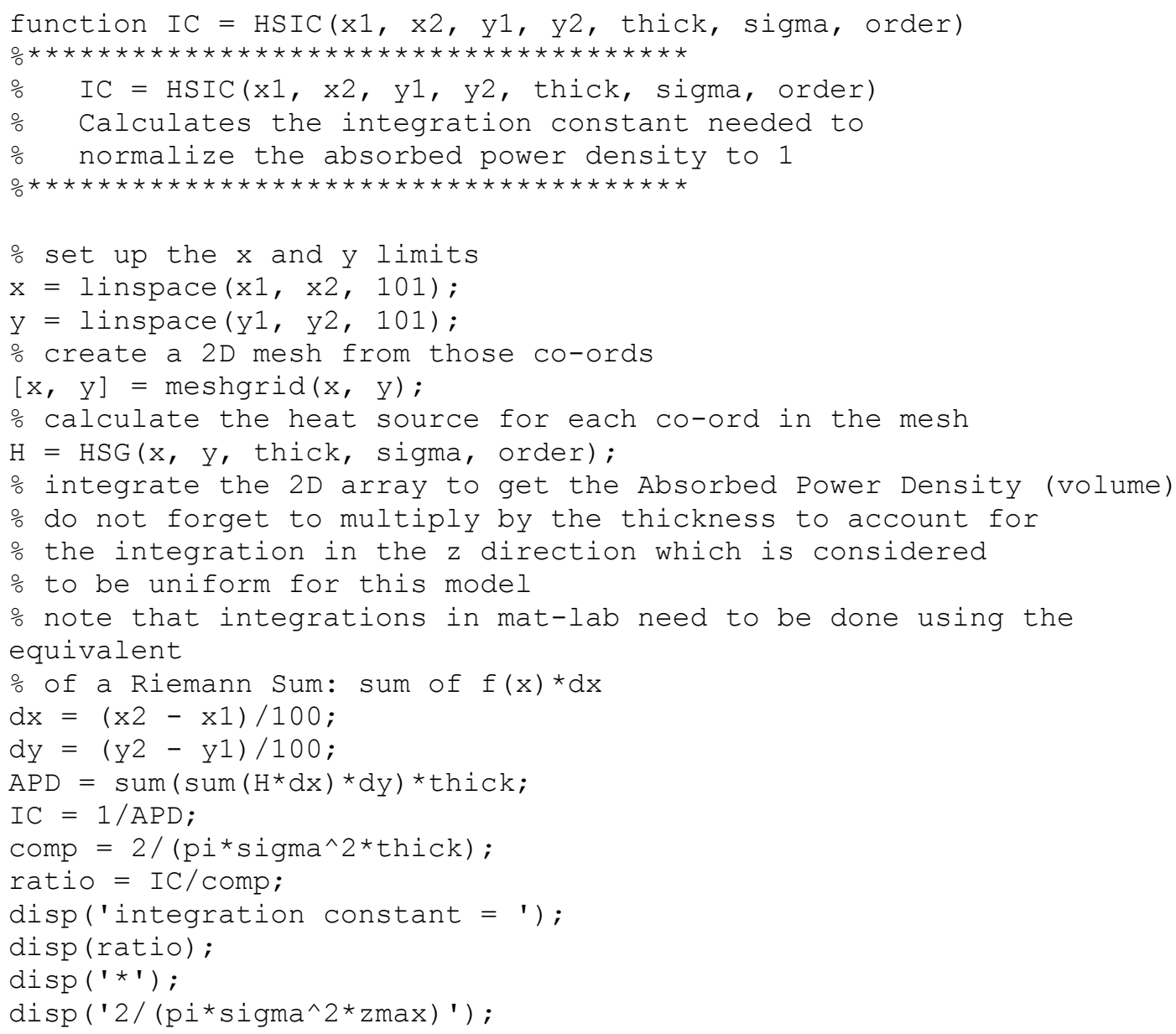




\section{Listing 3: HSG.m}

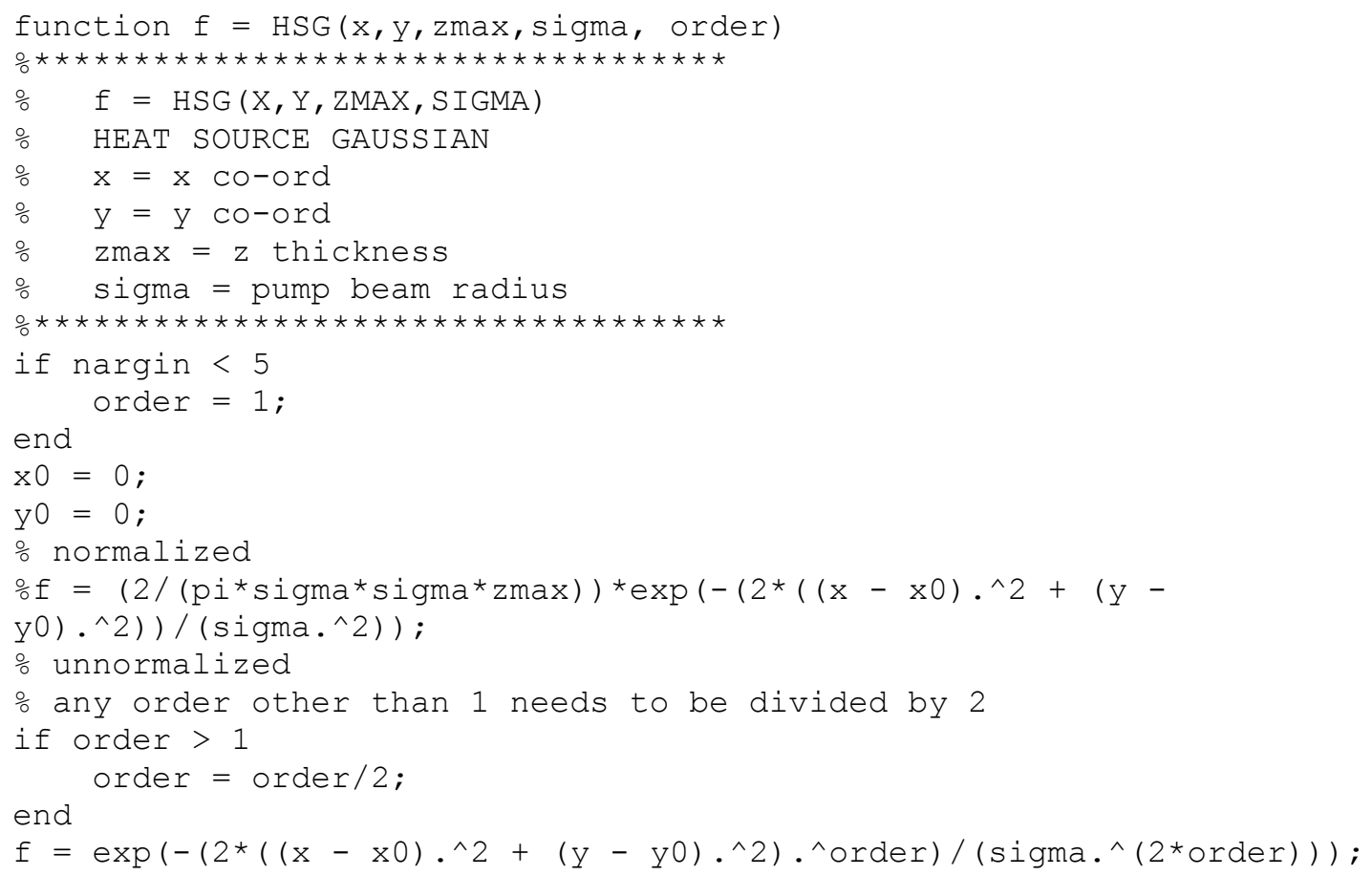




\section{Listing 4: HSICR.m}

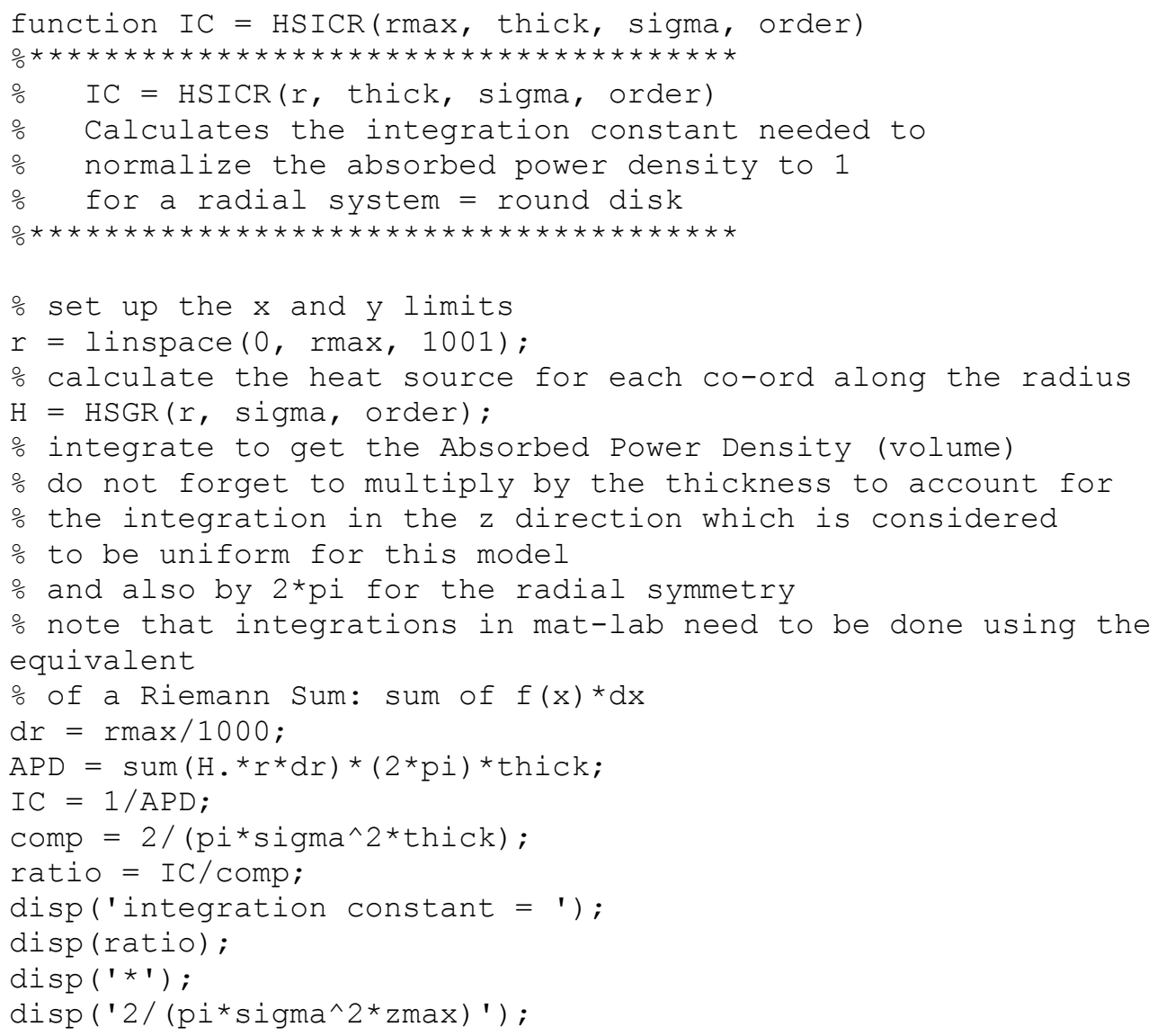




\section{Listing 5: HSGR.m}

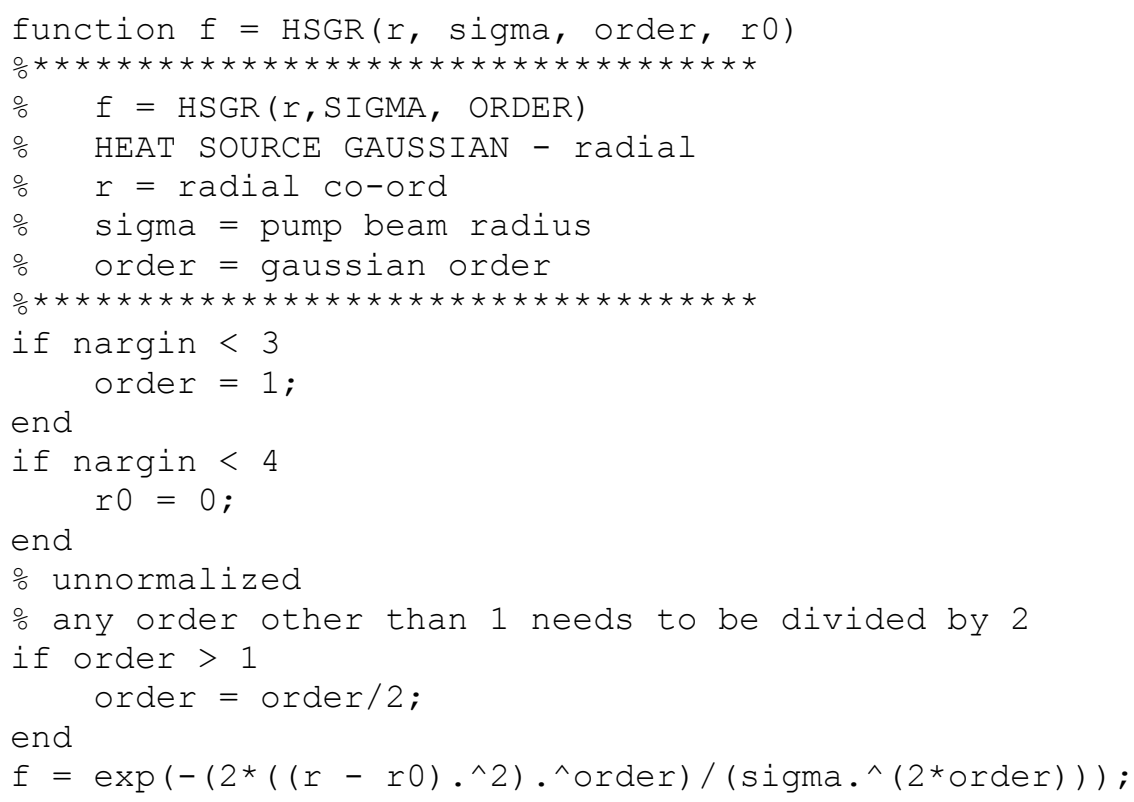




\section{Listing 5: gain_model3.m}

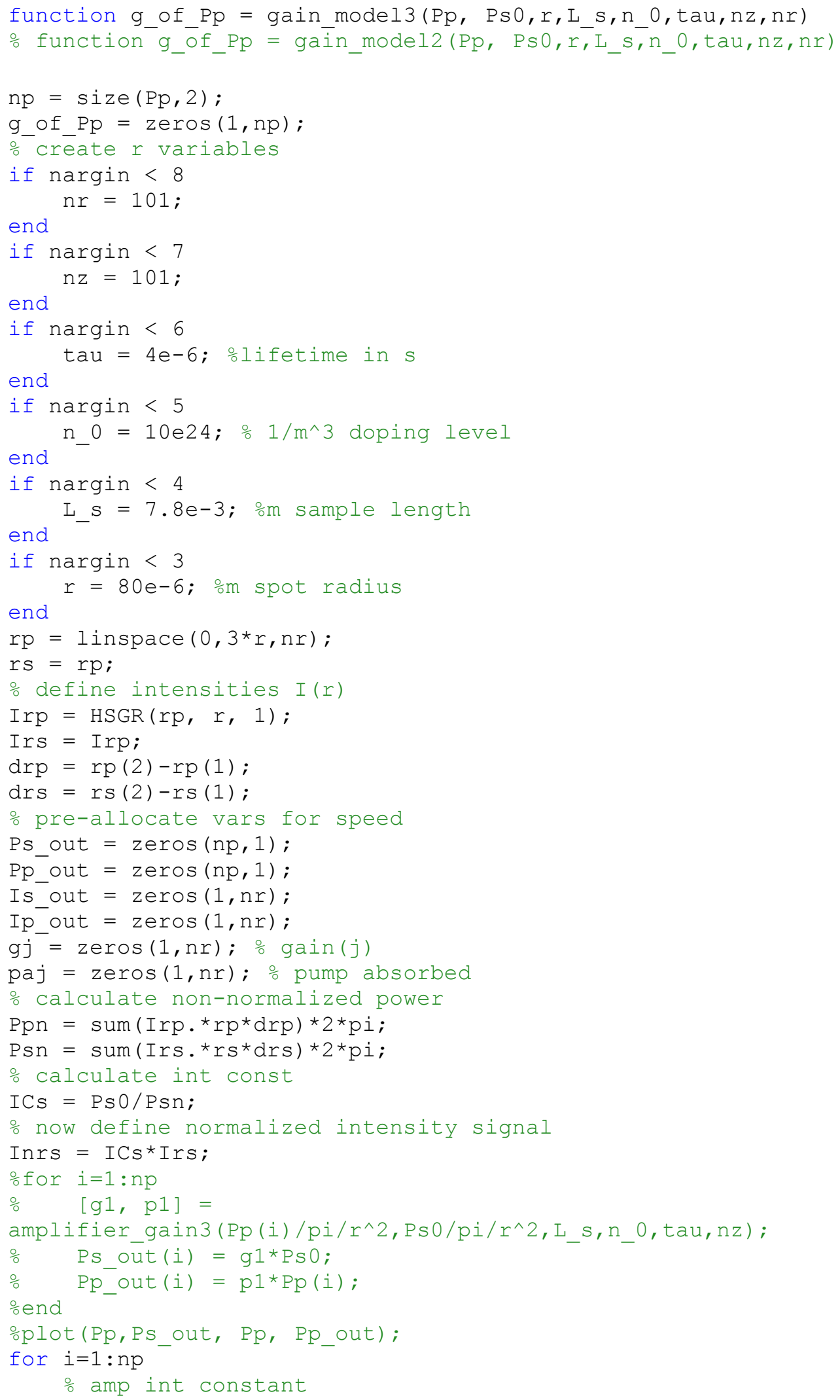




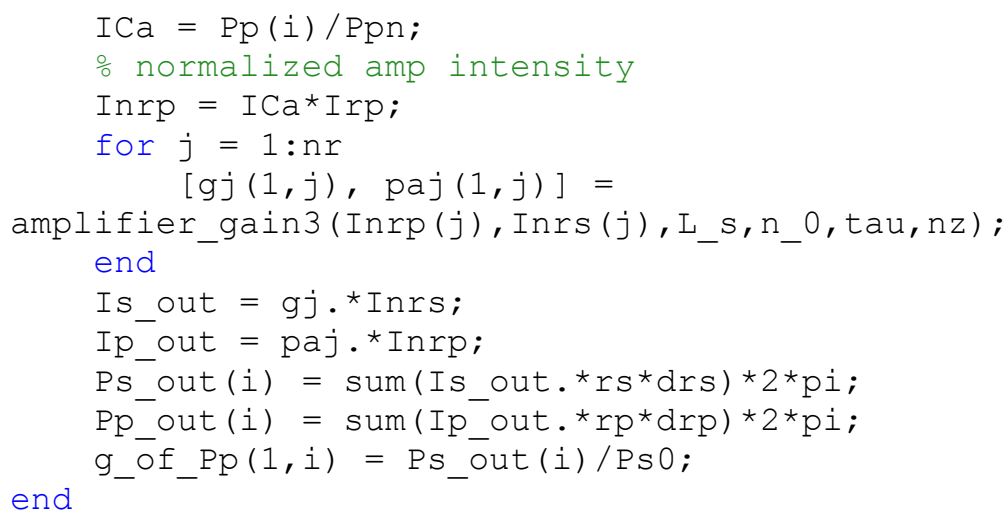




\section{Listing 6: amplifier_gain3.m}

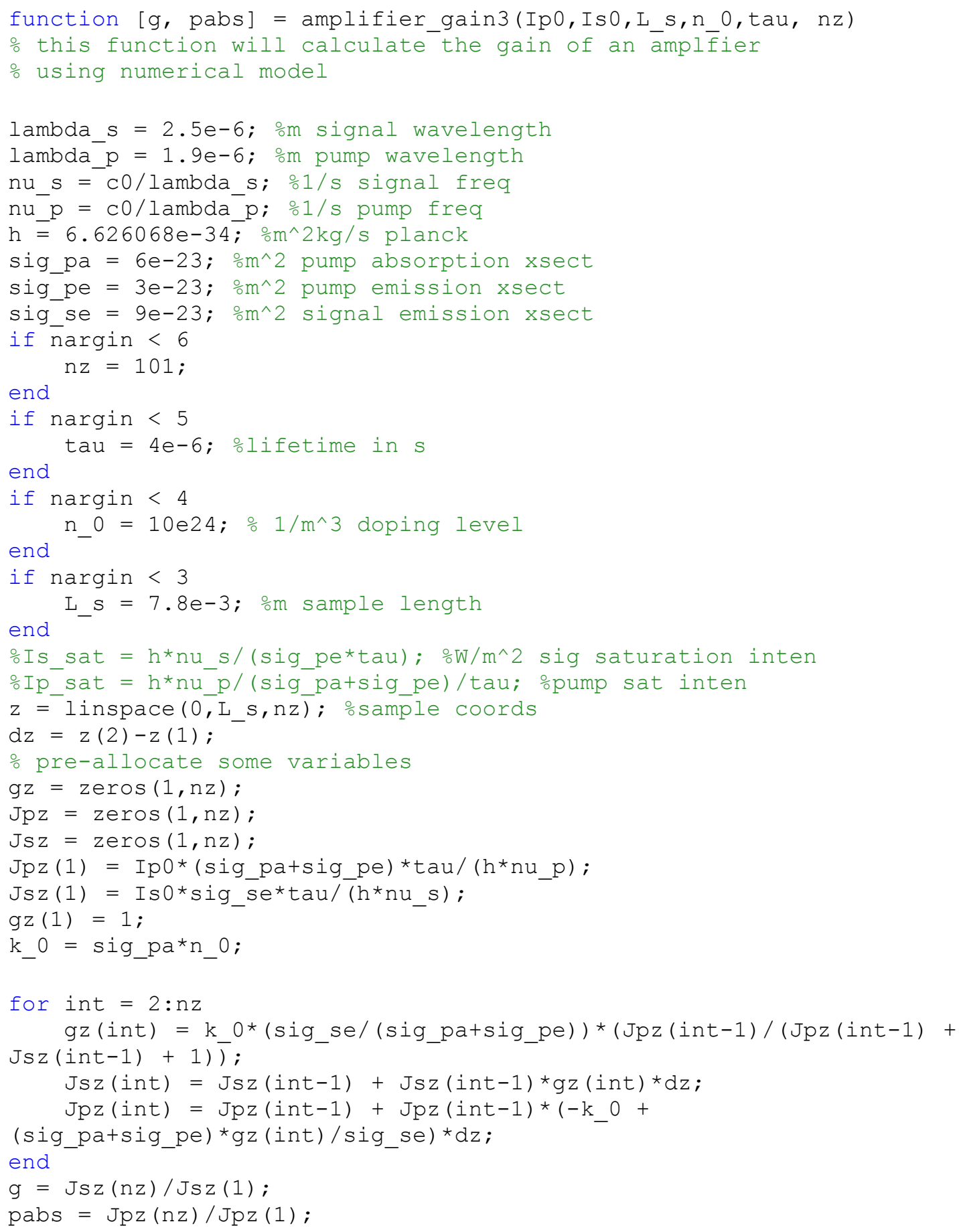




\section{Appendix 2}

\section{LabView Control Program for Laser and Power Meter Control}

A revised control program was written and tested for use with both IPG lasers and all Newport power meters (1918-C and 2935 models). This program allows for simultaneous control of both lasers independently while collecting statistical data from the power meter. Data collected from the power meter can be saved for use in MatLab or other data processing program. The status of both lasers is displayed in real time to alert the user to any problems.

The LabView programming environment is an icon-based system. Typically programs are meant to execute once or continuously in a loop. For the command-based terminal language of the both the IPG lasers and the Newport power meters, an evendriven architecture is more suitable so that queries are not constantly being sent to all instruments. To accomplish this with LabView, it is necessary to use the LabView event structure to respond to button pushes and similar events.

The $150 \mathrm{~W}$ laser (Figure 36) is controlled by entering changes to percent current (from 13 to $100 \%$ ). The $40 \mathrm{~W}$ (Figure 37) laser is controlled by entering either changes to power level (from 1-40 W) or changes to current $(0-\sim 5000 \mathrm{~mA})$. The status display block for either will show any status changes reported by the laser. 


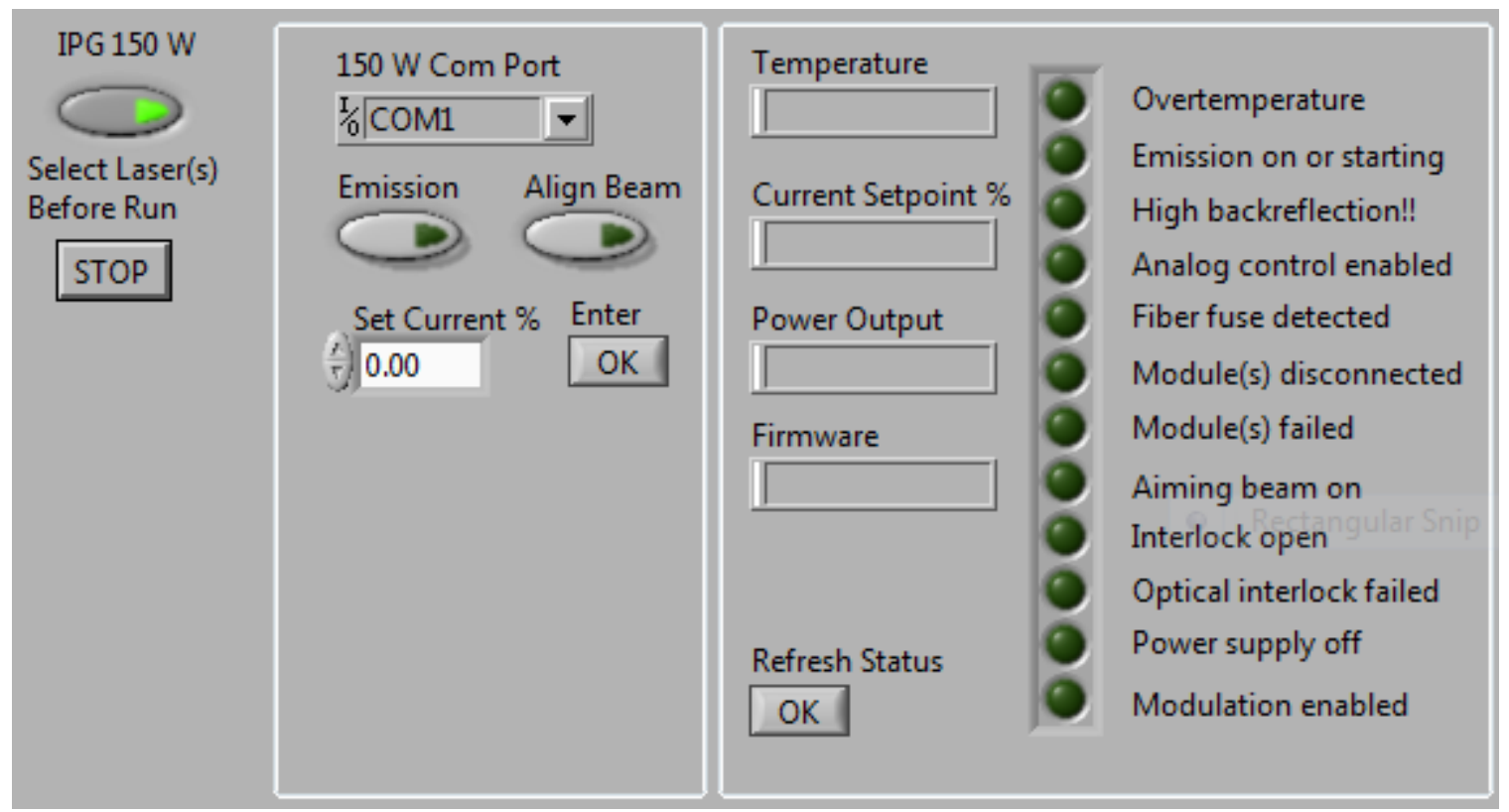

Figure 36: Control panel for IPG 150W Tm:Fiber laser control

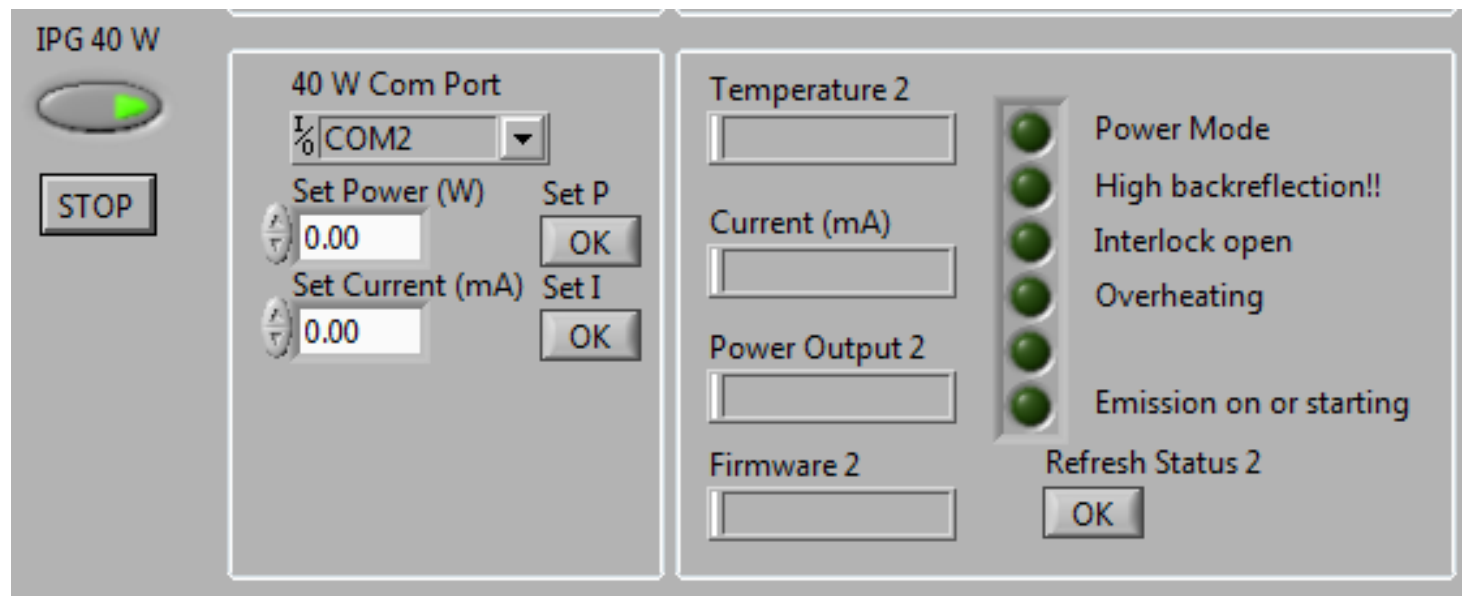

Figure 37: Control panel for IPG 40W Tm:Fiber laser control

The control block for the power meter (Figure 38) allows for control of either a 1918C single channel power meter or a 2935 dual-channel power meter. The control panel can be used to zero either channel and take set numbers of data points at a given rate and then store the mean, min, max and standard deviation. It should be noted that the rate is in approximately $\mathrm{kHz}$ (i.e. 10000 pts at "1" equals about 10 seconds). The right side of this panel can be used to run automated scans by starting one of the lasers at a given current (again $\%$ for the 150 and $\mathrm{mA}$ for the 40), waiting a set amount of time, then 
taking a given number data points, stepping by a given step, until reaching the ending current. This is useful for automated slope efficiency measurements which remove human variations from the results.

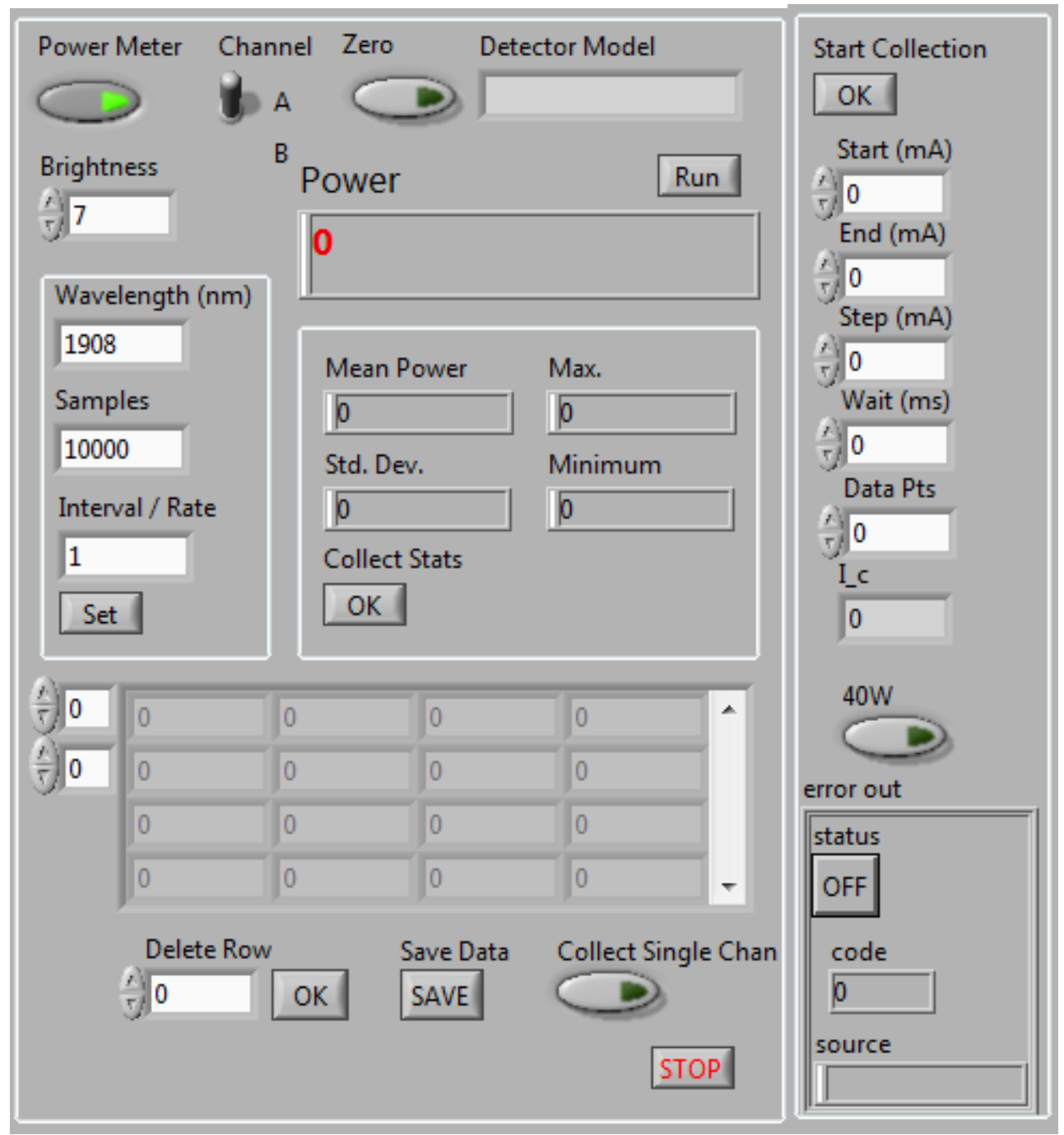

Figure 38: Control panel for power meter automated data collection (left) and for automated laser control with data collection (right) 


\section{Appendix 3}

\section{Z-Cavity Alignment Procedure}

The procedure described below is one used to align the single-leg Z-cavity described in Chapter 5. This same procedure was adapted for use with the other MO cavities. In Figure 39 below, M1, M2 are 50 mm ROC mirrors, DC is a flat in-coupler, all of which are HR@2.5 $\mu \mathrm{m}$, AR @ 1.9-2.1. L1 is a $50 \mathrm{~mm}$ focal length lens, dl, d2 and d3 are $25 \mathrm{~mm}, \mathrm{~d} 4$ is $160 \mathrm{~mm}$, and A1-A5 are adjustable aperture irises. The $1.907 \mu \mathrm{m}$ pump comes into L1 co-aligned with a $5 \mathrm{~mW}$ Helium Neon alignment laser. L1, M1 and M2 are on translation stages.

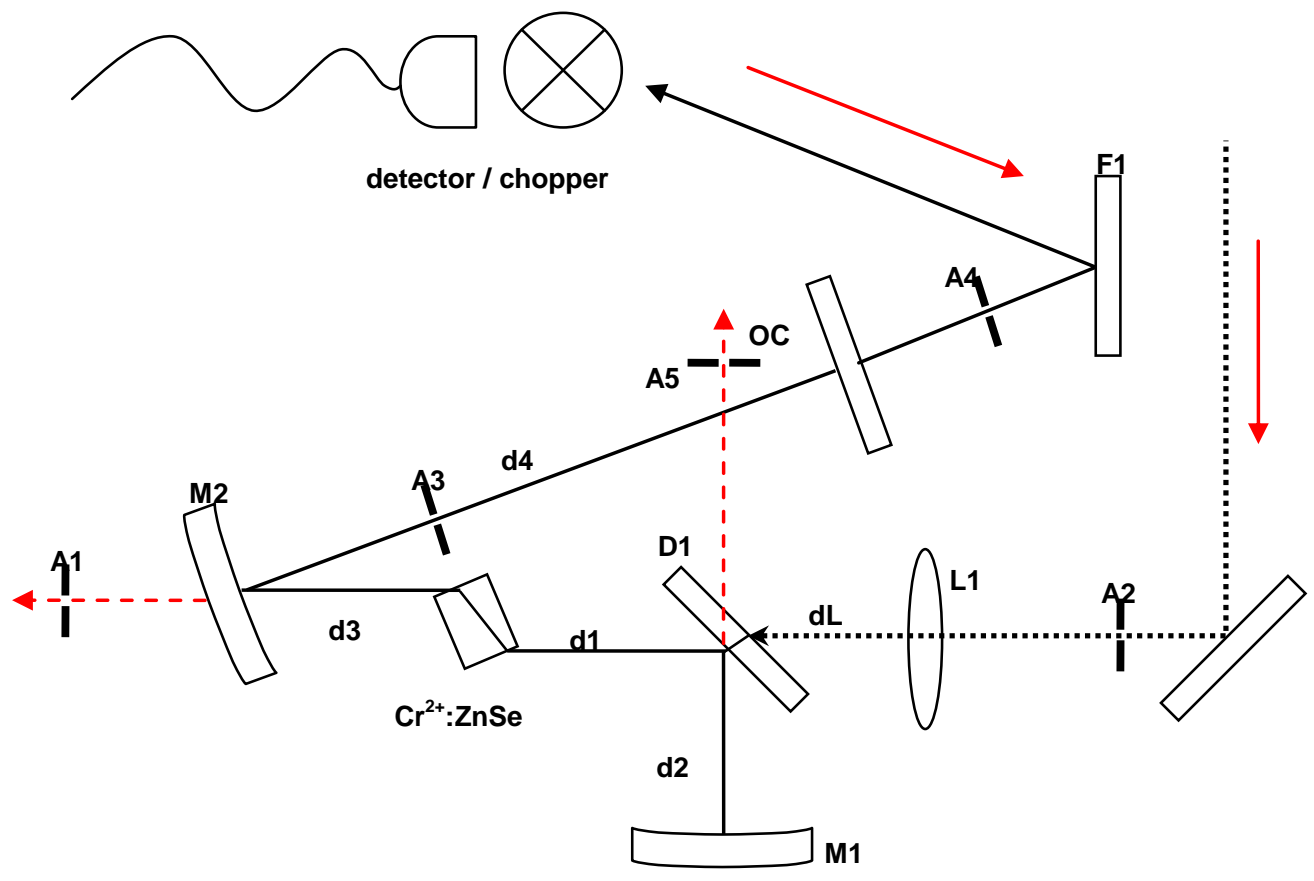

\section{Figure 39: High power Kogelnik cavity}

The following rigorous alignment procedure was developed with input from collaborators at UAB and used to align the laser cavity:

1. Remove all optical elements from the optical table. Ensure that the HeNe beam is parallel to the optical table and is aligned with the pump beam. 
2. Install the input coupler (DC).

3. Position an iris aperture (A1) $~ 150 \mathrm{~mm}$ from the proposed location of the pump lens and center it with the HeNe beam.

4. Add another iris aperture (A2) before the pump lens position and center it with the HeNe beam.

5. Install the pump lens (L1) and align it vertically and horizontally to make sure that the propagated $\mathrm{HeNe}$ beam is centered on the iris aperture A1.

6. Install an iris aperture (A5) and center it with the reflection of the HeNe beam from the internal surface of the flat pump mirror. The in-coupler DC causes significant diffraction. The iris should be aligned with the second brightest reflected spot.

7. Install the laser crystal and align it to the Brewster angle (67 degrees for zinc selenide). This can be done by using a polarizer on the HeNe and finding the minimum reflection point. Triangulation can also be used. The laser crystal should be mounted on a three axis stage.

8. Even a very weak HeNe beam should be visible after propagation through the crystal, so ensure that the beam appears somewhere on iris aperture A1. It will be displaced from passing through the crystal.

9. Install mirror M2 and adjust it to the appropriate angle $\left(15-20^{\circ}\right)$. Displace the mirror vertically as necessary until the reflected HeNe beam $\mathrm{d} 4$ is horizontal.

10. Adjust the distance $\mathrm{d} 3$ until the reflected $\mathrm{d} 4 \mathrm{HeNe}$ beam is collimated. This indicates optimal distance for $\mathrm{d} 3$.

11. Use two iris apertures (A3, A4) to indicate the path of the $\mathrm{d} 4$ beam leg. Care should be taken with these apertures to not interfere with the cavity mode while leaving room for the outcoupler OC.

12. Align a second $\mathrm{HeNe}$ beam counter-propagating along $\mathrm{d} 4$ through apertures $\mathrm{A} 3$ and $\mathrm{A} 4$. If the reverse $\mathrm{HeNe}$ beam is aligned correctly with $\mathrm{d} 4$, you should also observe its collimated spot centered at the second iris aperture A2.

13. Install the end mirror M1 and align it so that the reversed HeNe beam, reflected from M1 is centered at the aperture A5. This is the hardest spot to align in the procedure. Turning out the room lights is helpful.

14. With no outcoupler, set up a suitable detector (like an extended range InGaAs detector) and a chopper at the output end of the propagation path, past A4 and off/through a filtering dichroic mirror F1 (to dump any pump signal). Turn on the pump laser and set it to 2-3 W. Observe the fluorescence signal on an oscilloscope triggered with the chopper signal.

15. Maximize the fluorescence signal at the detector by longitudinal adjustment of the pump lens and fold mirror M2.

16. Maximize the fluorescence signal at the detector by angular alignment of the end mirror M1.

17. Turn off the pump.

18. Install the output coupler. Use a highly reflective OC (95\%) for easier initial alignment. Use the retro-reflection of the reverse $\mathrm{HeNe}$ for preliminary alignment.

19. There is no step 19.

20. If the forward HeNe beam reflection from OC is visible through the cavity, align OC so that the reflected beam passes through the aperture A3.

21. Turn the pump laser on and set it to $5 \mathrm{~W}$.

22. Quickly scan the OC horizontally while slowly scanning it vertically until a lasing spike is seen in the fluorescence signal. 
23. Further align all other optical elements in a repeatable pattern, slowly decreasing the pump power after each alignment cycle, until the minimum lasing threshold is obtained.

24. If lasing could not be obtained on the previous step then the cavity is probably in unstable condition.

25. Turn the pump laser off and pre-align the OC again as in steps 18-20.

26. Turn on the pump and set it to $5 \mathrm{~W}$.

27. Move the fold mirror M2 closer to the crystal by a few millimeters.

28. Slowly increase $\mathrm{d} 3$ in small steps $(\sim 200-500 \mu \mathrm{m})$ and at each step shake the OC in two angular directions. The stable alignment of the laser cavity will eventually be found and lasing obtained.

29. Optimize the cavity to the minimum threshold by realigning all optical elements in a repeatable pattern.

30. Note that the distance $\mathrm{d} 4$ is not critical. It can vary from $30 \mathrm{~mm}$ to $100 \mathrm{~cm}$ without altering the cavity stability. 